\title{
Alphabetirates Derzeidnis
}

\section{berjenigen \\ Mitglieder des beutiden Juriftentages. roelde

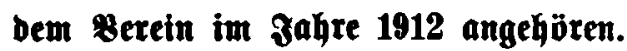

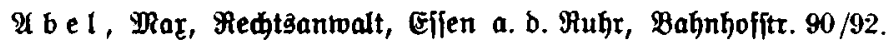

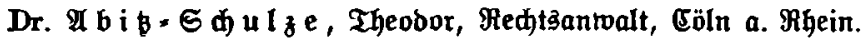

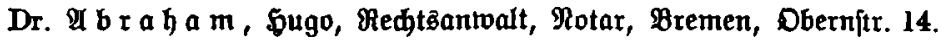

Dr. গ⿺ 一 Э̛ährịtr. 12.

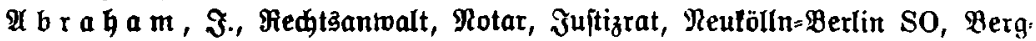
itrape 125.

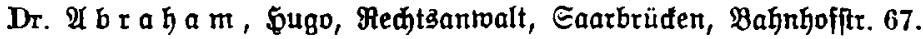

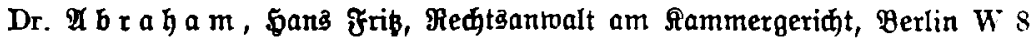
Jriebridiftr. 182.

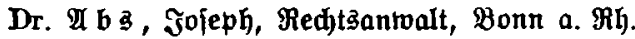

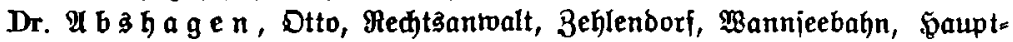
ftráge 1 II.

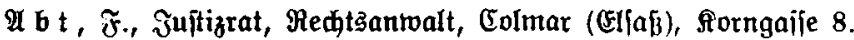

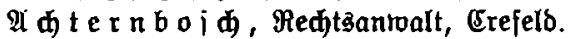

I $\delta$ a m , Fedtsantwalt, Rotar, Jujtizrat, Danzig.

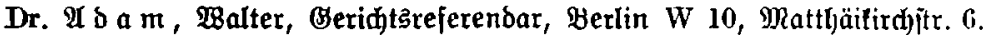

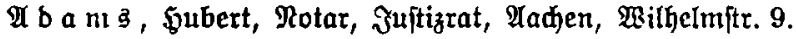

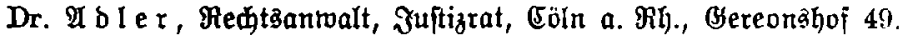

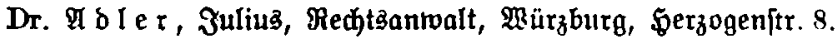

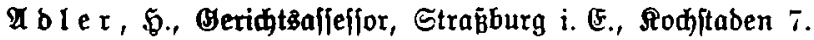

A b l e r , Siegfrieb, Redtsantwalt, Münden, Brienner[tr. 55 II.

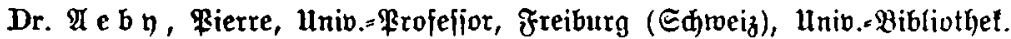

$\mathfrak{A} \backslash \boldsymbol{b} \in \mathbf{r} \mathrm{s}$, Rgl. Rotar, Erefeld, Ditivall 130 .

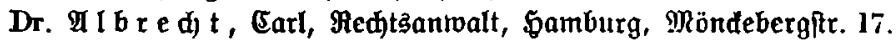

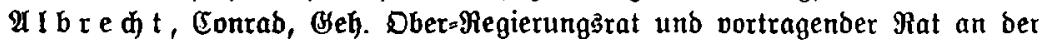

Oberrechnungstammer, Bot3̧bam, Eijenfartftr. 6.

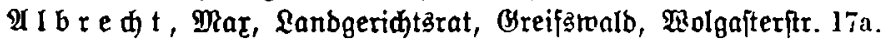

Dr. A I e r a n

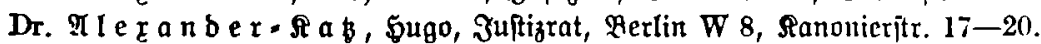

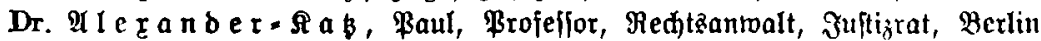

W 8 , Rillfelmitr. 52. 


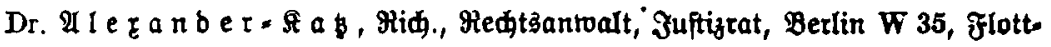
rellitr. 1.

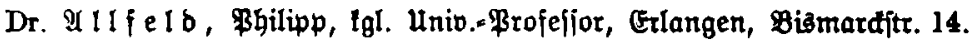

Dr. $\mathscr{I}$ I $0 \pi$ a

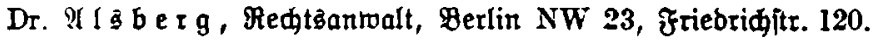

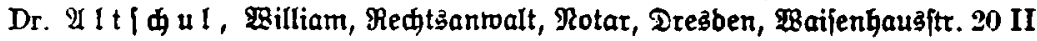

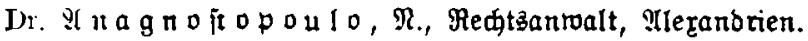

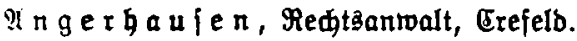

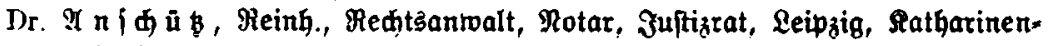
fitráe 3.

Dr. Anspacher, Luigi, Arrocato, Milano, 26 via Orifici.

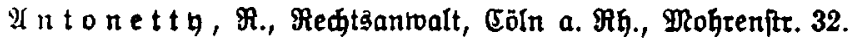

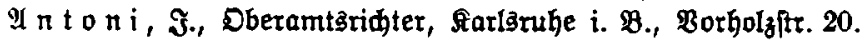

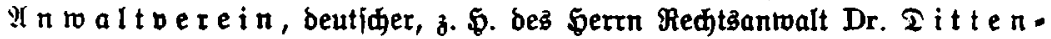
berger, Leibzig, Sdreberftr. 3.

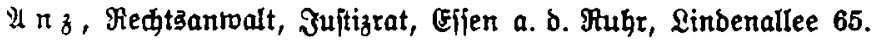

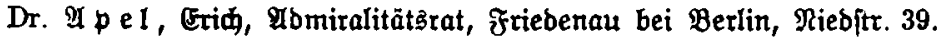

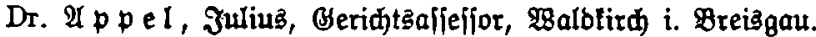

Dr. $\mathfrak{A} \mathfrak{t}, \mathfrak{M a r}, \mathfrak{B r o j e i j o r , ~ B e r l i n ~ N W ~ 4 0 , ~ I n ~ b e n ~ B e l t e n ~ 9 a . ~}$

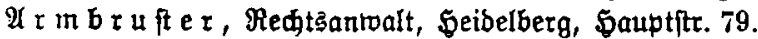

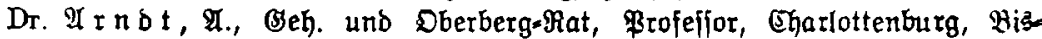
itarfitit. 66.

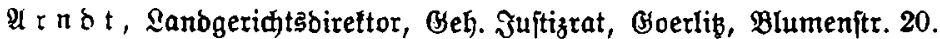

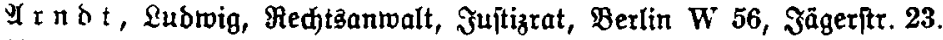

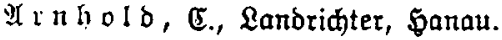

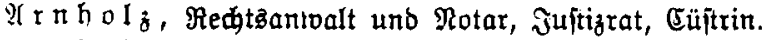

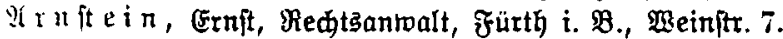

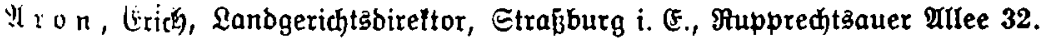

I r 0 n $;$ o $\mathfrak{h}, \mathfrak{R e d}$ tsantwalt, Bromberg.

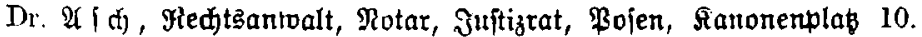

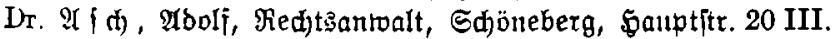

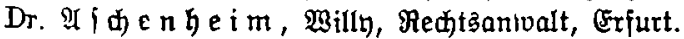

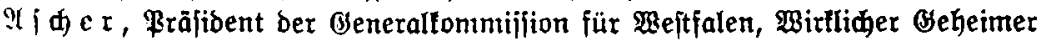
Ober=Regierungżrat, Müniter i. Wa.

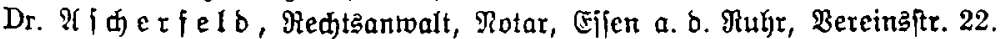

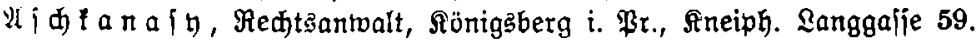

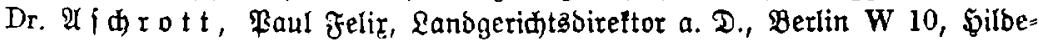
brandtitr. 8 .

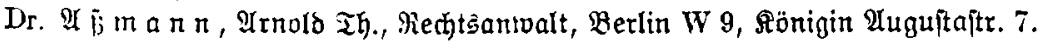

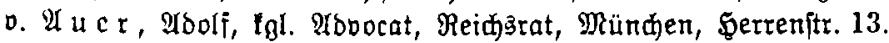

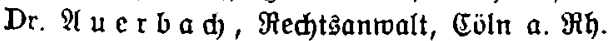

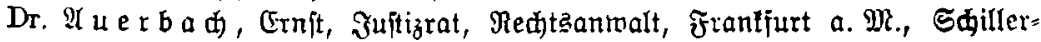
jtrafje 22.

Dr. : แ e r $\mathfrak{b}$ d , Red)tzantualt, Bromberg.

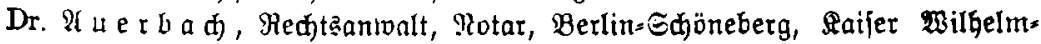
plats 4.

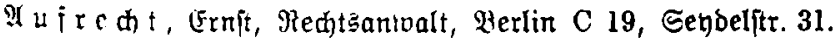




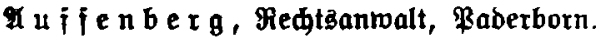

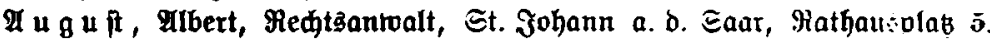

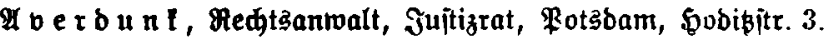

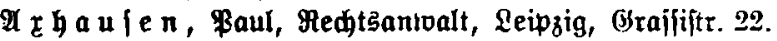

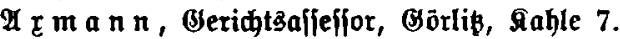

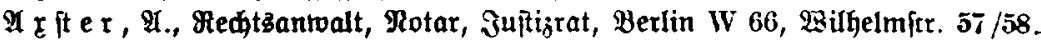

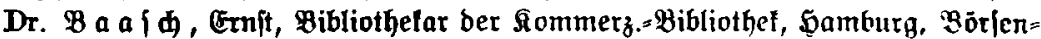

भrtaben.

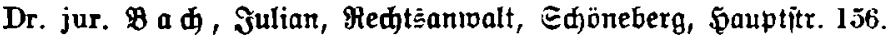

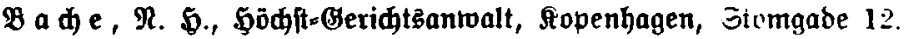

Dr. B a de $\mathrm{t}$, Guftav, Redtsantwalt, Mannheim.

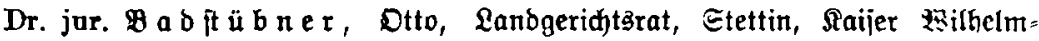
Ptráвe 76.

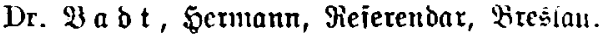

Dr. jur. $\mathfrak{B} \mathfrak{a} \mathfrak{h} \mathfrak{r}$, Fugen, Budhänbler, Berlin=⿰riebenau, (Evajtr. 4.

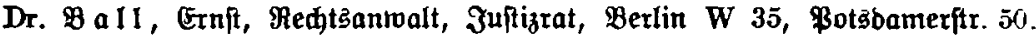

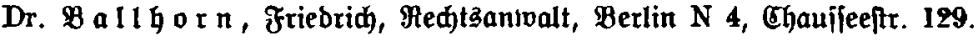

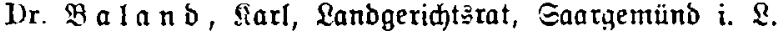

Dr. $\mathfrak{Y}$ a m be r $g$, ફugo, Pedtsanwalt, Berlin W 8, Behrenftr. 14/16.

Dr. v. $\mathfrak{B}$ a r, 2., Beh. Jultizrat, Broj., Böttingen, Gerzberger (Eqaujfee 25.

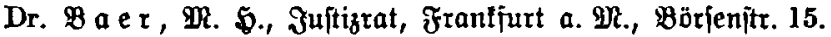

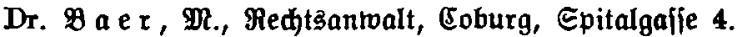

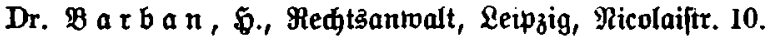

$\mathfrak{B}$ a r б e w i

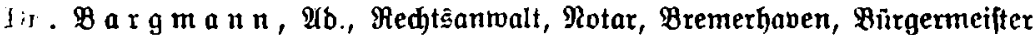
Emioitr. 74.

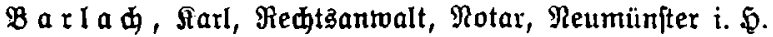

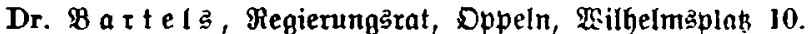

Dr. Sartel s, Dtto, Juftizrat, Gaifel.

Dr. B a rt $\{$ d, Georg, Redtäantoalt, Brieg, Bez. Breßlau, Bollitr. 8,9

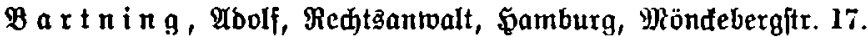

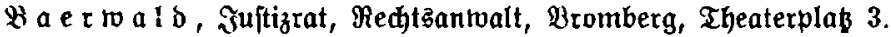

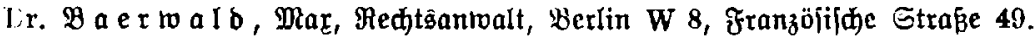

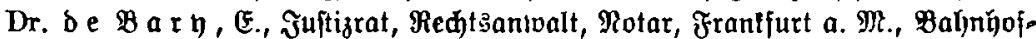
frafe 2.

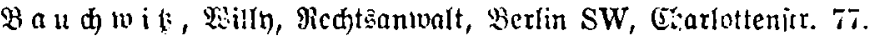

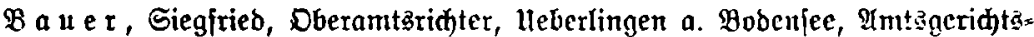
gebäube.

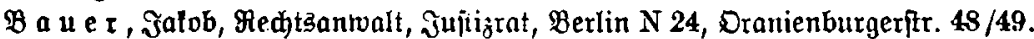

Dr. $\mathfrak{B} \mathfrak{a} \mathfrak{u}$, Georg, Mechtsantwalt, Berlin W 30, Mobjtr. 69.

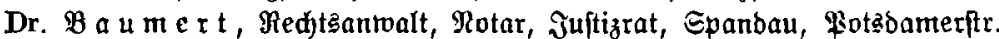

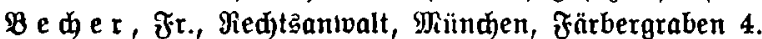

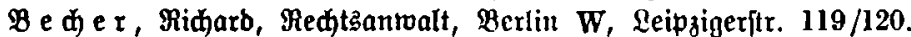

Dr. Be de r, Robert, Redtsanmalt, Geh. Juftizrat, Düfieloori.

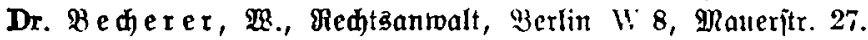

Dr. Be đ, E., Ređtzantwalt, Eannitatt.

Be de $\tau$, Beorg, Redttzantoalt, Berlin No 18, (Brop̉e Frantfurteritr. 102. 


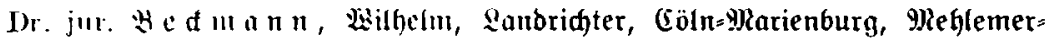
itraje 6 .

J)r. Beer, (E., Hed)tsamualt, Herlin W 64, Hehrenjtr. 24.

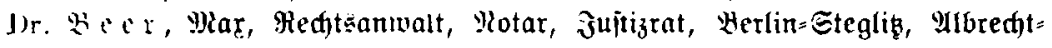
itratic 12.

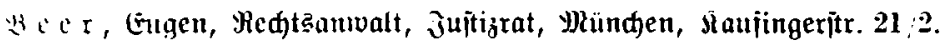

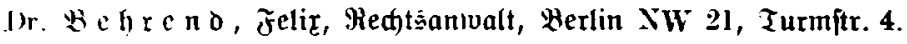

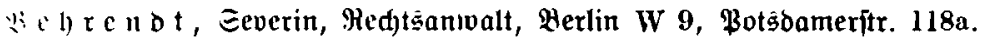

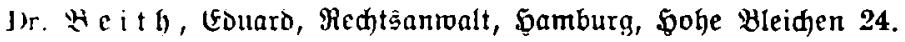

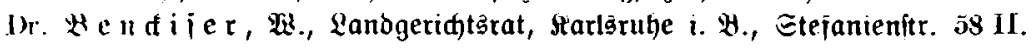

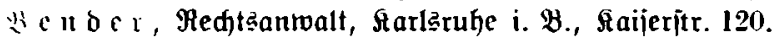

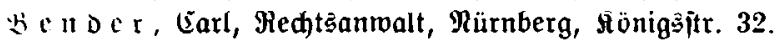

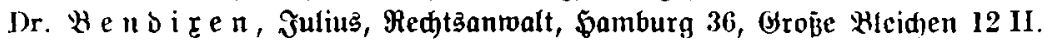

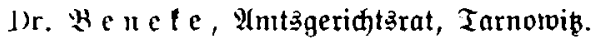

B e $n$ i $\varepsilon$ y III, Redtsanualt, Eannoper.

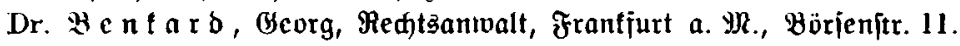

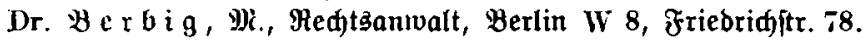

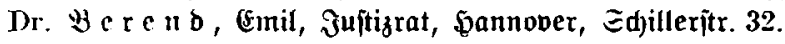

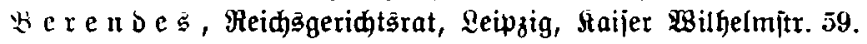

Dr. $B \mathfrak{e} g$, Geridtżafieffor, Sharlottenburg, Berlineritr. 162.

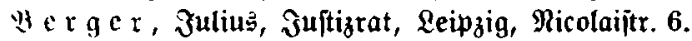

Dr. B i r i

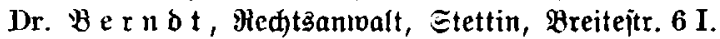

Dr. Herne, Redtşanralt, Berlin W 8, Behrenitr. 27.

Dr. S' e $x$ u he $\mathfrak{i}$ m, Seinrid, Redtsanmalt, Mannheim.

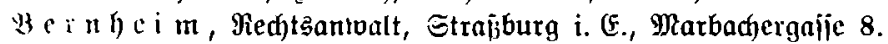

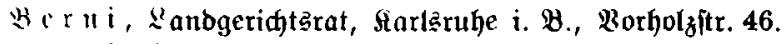

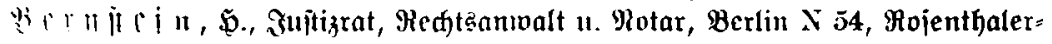
Etrayse 4.3 .

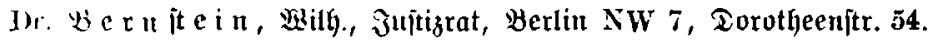

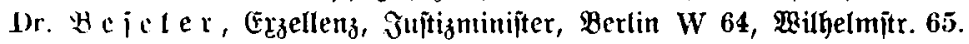

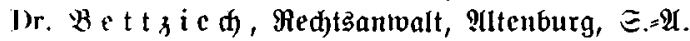

Wi $\mathfrak{e} b$, Friebrich, Qandgerid)tšrat. Meiblingen $\mathfrak{b}$. Stuttgart.

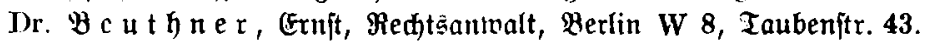

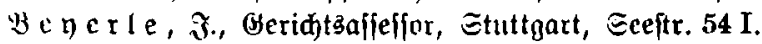

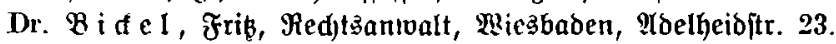

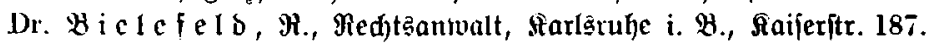

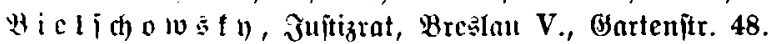

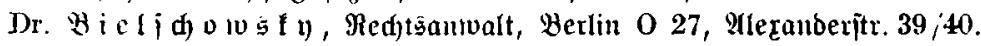

Hi c nert, Hedtsanualt, Jotar, Rübed, Breiteftr. 4I I.

D. Dr. B্ icrling, Ger. Juitizrat, Brofeijor, Greifätualo.

Dr. \&i i e r m a n n, Projejior, (jeh. Juftizrat, balle a. S., Jajanenitr. 6.

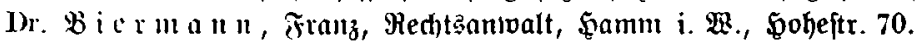

1)r. Wi it o c r, J., Frofcfior, Erlangent.

Dr. \&i in g, Medtsanualt, Cöln a. Mh., Tielhans.

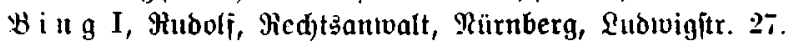

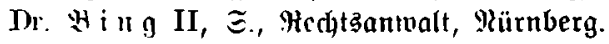




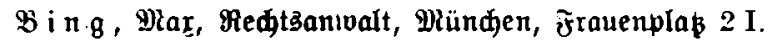

Dr. $\mathscr{B}$ in $\mathbf{t}$ ing, Redtsantwalt, Motar, Lanbaberg a. Sa.

Dr. Bi r t me yer, Beh. \$ofrat, Brofeijor, Mänden, Diterinaloịr. 95.

$\mathfrak{B}$ i t t a $\mathfrak{R}$. A., Sujtizrat, Breßlau XIII, fömeritr. 23/25.

Dr. B i t t e r, Redtsantwalt, Rotar, Jujtizrat, Fiel, Goitenaucrīt. 4.

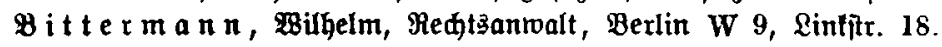

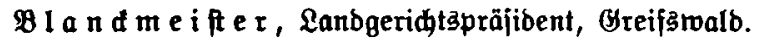

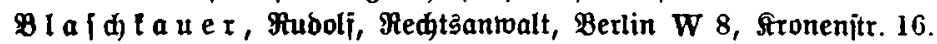

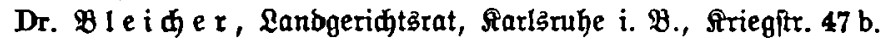

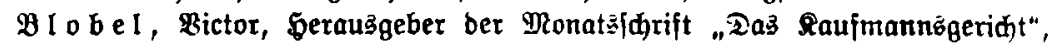
Samburg, Golftentuall 4.

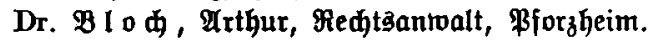

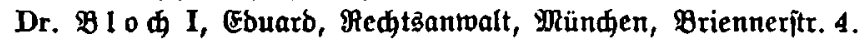

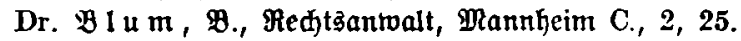

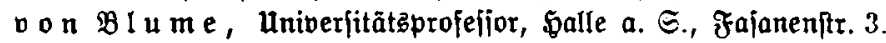

Dr. jur. $\mathfrak{B} \mathfrak{I}$ ũ m $\mathrm{I}, \mathfrak{B r}$. Rotar, Pforzheim.

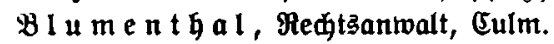

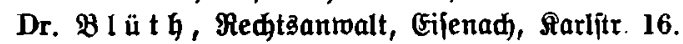

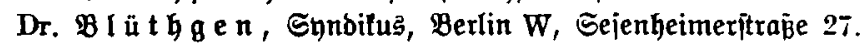

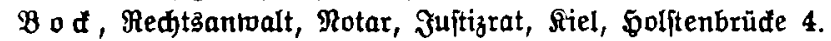

B o d, Redtanantwalt, Magbeburg, Breiterveg $21 / 22$.

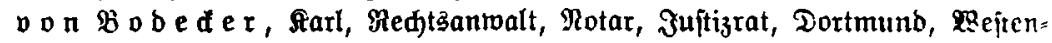
hellweg 17.

B o be nhe imer, Redtsantualt, Darmitadt, Bismarditr. 81.

Dr. jur. B o b I a e n $b \in \mathbf{r}$, Gilvio, Redhtzantwalt, Berlin W 9, Fot3tamer= jtrā̧e 134b.

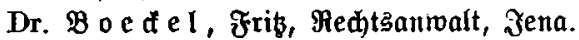

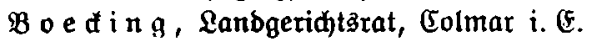

Dr. $\mathfrak{B}$ o ge n g, B. A. E., Serlin W 30, Mojenheimeritr. 17.

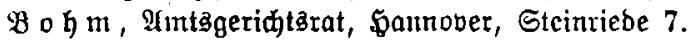

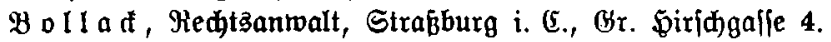

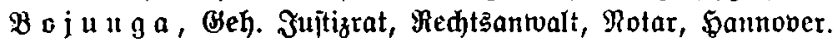

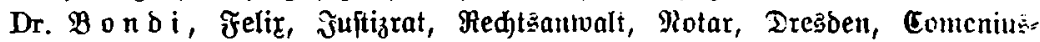
ftraje 33.

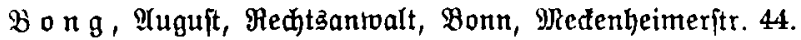

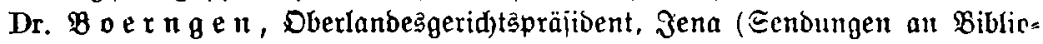
thetzoerwaltung Dez Dberlanbesgeridts Jena.)

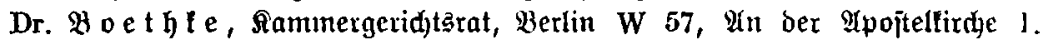
Bo et $t$ ger, Ernft, Bedtsantoalt, Buttitaedt i. Th., Bahnhofätraje.

Bo e o vo, 1. Staatzantvalt, Soezlin, Sarjigerftr. 11/15.

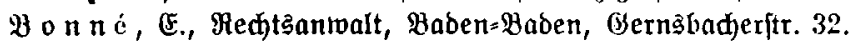

Dr. $\mathfrak{B}$ ord) e $\mathbf{s}$ II, Rechtşantwalt, Eelle.

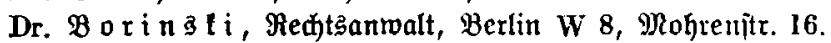

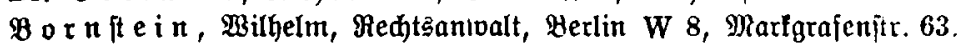

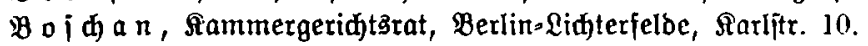

Dr. $\mathfrak{B}$ ote, $e$., Redtsantwalt, Güftrotw i. Mieđll.

Dr. jur. et phil. $\mathfrak{B}$ o venfiepen, Sonbridter, Giel. 


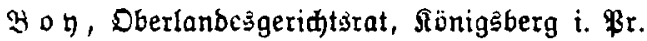

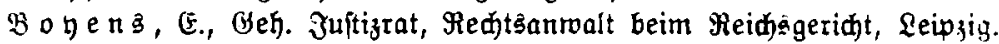

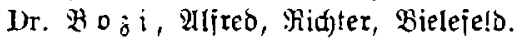

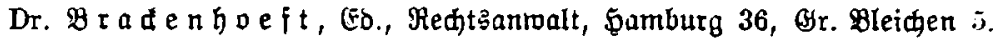

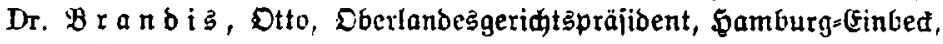

Şagenau 78.

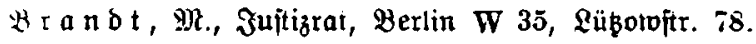

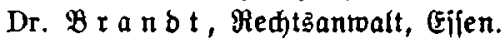

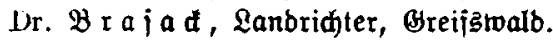

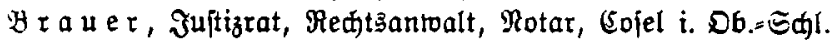

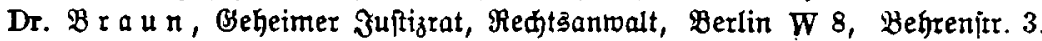

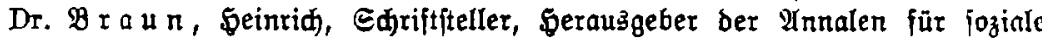

Politif unb Bejełgebung, Behlenbori, Exlentweg.

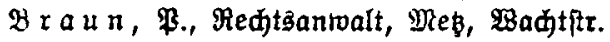

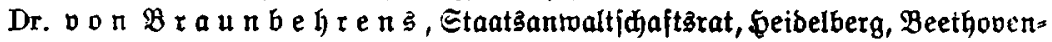
itrá̉e.

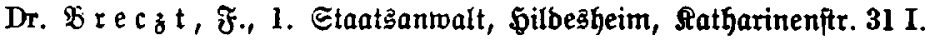

Br e i sli $i$, Redtsartwalt, Danzig, Gunbegafie 22.

B $x$ e i t, Mat $\mathfrak{M}$., Redtsantwalt, Reipzig.

Dr. Or r e it, James, Redtsantwalt, Dreşben, Elijenjtr. 10.

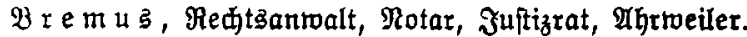

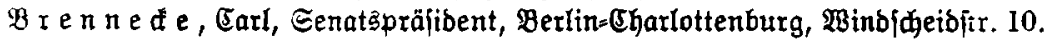

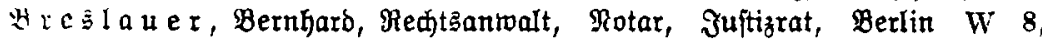
Mlohrenftr. 51.

Dr. $\mathfrak{B}$ r $\boldsymbol{n} \mathfrak{l}, \mathfrak{L} .$, Jultizrat, Rotar, Mayen i. Rhlb.

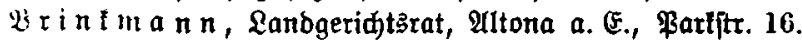

Si $x$ int in $a n n=\mathfrak{B}$ ondi, \$ans, Referendar, Bibliothelar bes jurijtijden

Ecmintară ber Sịl. Univeriität Berlin, Berlin=Brunervalb, פarm= brututerjtr. 8.

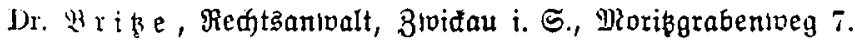

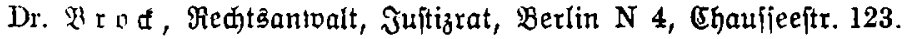

Dr. W y b b $i$, Beorg, ßrofeffor, Salle a. S., Reidarbjtr. 17.

Dr. 2ir o b ni B, Redtsanmalt, Berlin SW 68, Bimmeritr. 21.

B r o w n, Ulbert, Lanbgeridtšrat a. D., Charlottenburg, Grolmanjtr. 36.

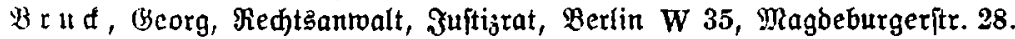

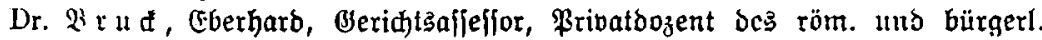
Ređht: an ber Univerjität Bres̆lau, Auenftr. 14.

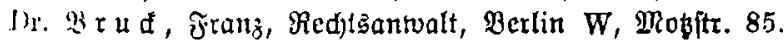

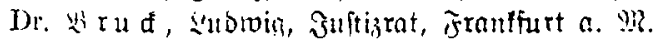

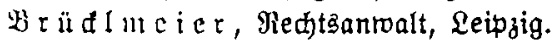

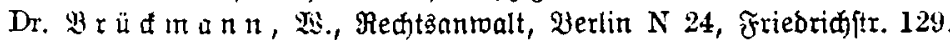

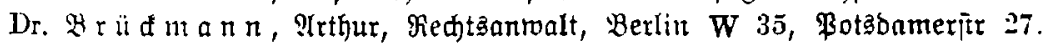

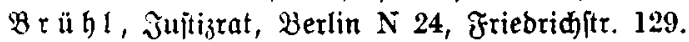

Dr. Br u n ner, Seintid, Wirflicher Geheimer Mat, Frofeffor, Erzellenz, Bscrlin W 62, sutherftr. 36 .

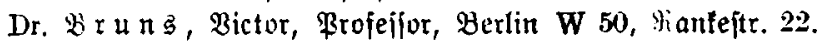

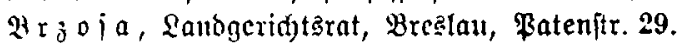




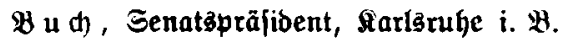

$\mathfrak{Z}_{\text {u }} \mathrm{ta}$, Redtzanwalt, Rotar, Juftizrat, Charlottenburg, Berlineritr. 124.

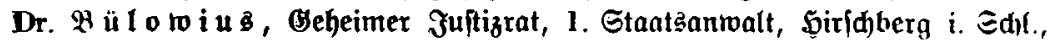
Stonzborferfitr. 9.

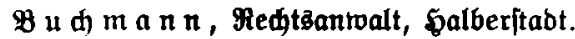

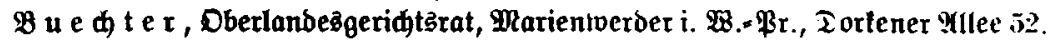

Dr. $B$ u m te, Lanbridter, Danzig $=$ Langfubr, Johannis̄thal Ip.

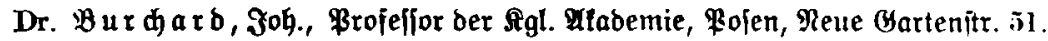

$g$ ü r g el e, Lanbridter, Яonip.

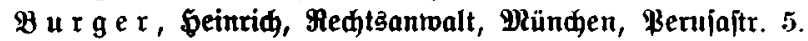

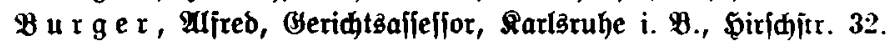

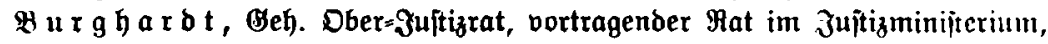
Eharlottenburg, Berlinerftr. 23.

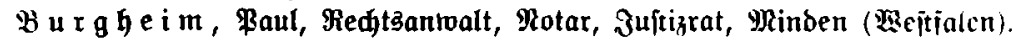

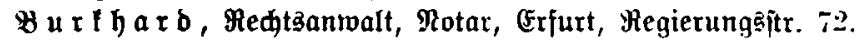

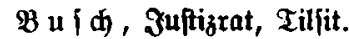

\&u id), Otto, Mifefior, Bera (Meub).

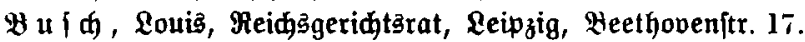

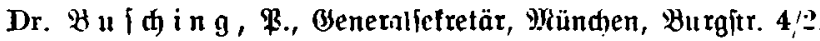

$\Re$ ï $t$ e, Reфtsantwalt, Crefelo.

Dr.

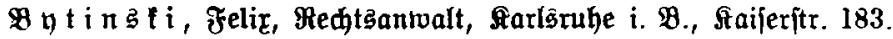

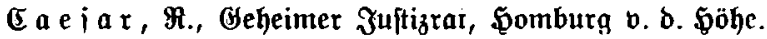

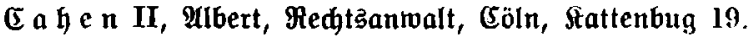

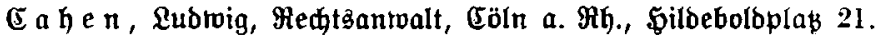

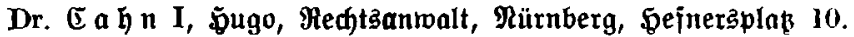

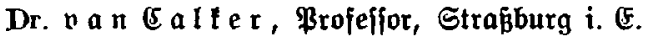

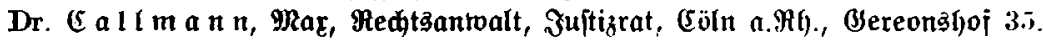

Dr. Ea nnebt, Carl, Medhtsantwalt, Potar, Berliu sil 11, Puthaltftr. 1?.

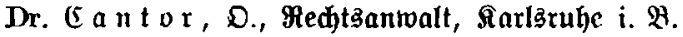

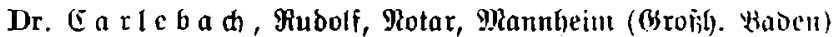

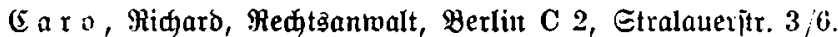

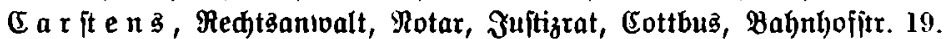

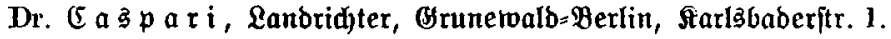

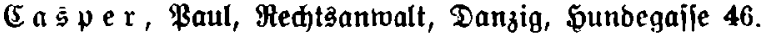

(S a i i e 1, âlifed, Medtananwalt, Berlin W 8, Mohrenjtr. 61.

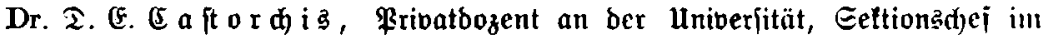
३uftizminifterium, 2tthen, Ebuard Lowittr. 12.

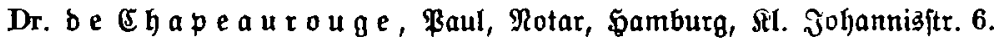

Dr. von Eetto, Earl Freiferr, Direltor b. Bayerifden Lanbrirtidafts= bant, Bräfibent bes Bayerifden Ranbrirtidaftärata, Münden, $\mathfrak{B a g}=$ mulleritr. 18.

(5h⿻ Cho b z i з ner, Mac, Redtsantwalt, Berlin NW 7 , Unter ben Rinben 42.

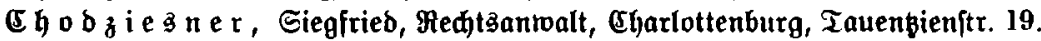
Dr. (E h or ma n n, Meinharb, Redtaanroalt, Zuftizrnt, Mülhnulen i. E., Ђoffnung?tr. 9. 


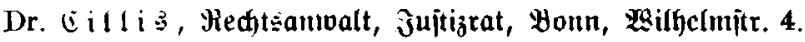

Ei i r vu, Jujtizrat, Danzig, Sunbegajie 94 I.

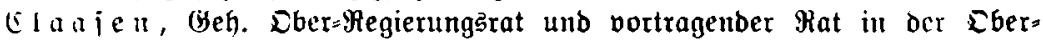

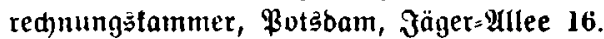

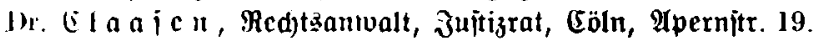

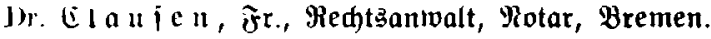

(i) i $\mathrm{m}_{\mathrm{c}} \mathrm{nt}$, Ebibarb, Lanbridtcr, Mlagbeburg= Subenburg, Weitentitr. 31 .

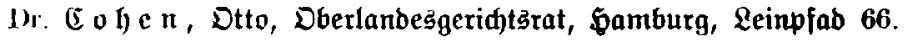

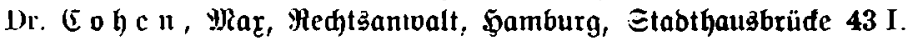

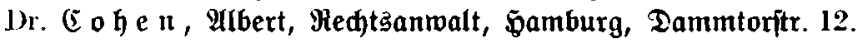

Jr. (E o h $n$, Mllired, Med)tsanwalt, Samlurg, Meuerwall 75 .

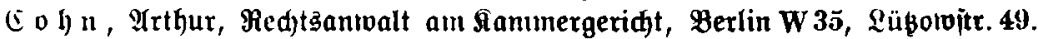

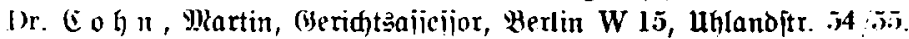

Dr. $\mathfrak{C}^{\circ} \mathfrak{h} n$, Wrartin, Medtsanuvalt, Herlin $W 10$, Draleitr. 1.

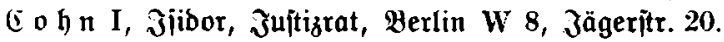

1)r. (E o h n, Lubwig, Beheimer 3uftizrat, Breşlau I, Zauentienjtr. 8/10.

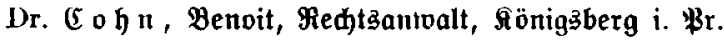

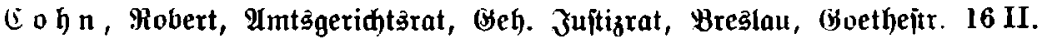

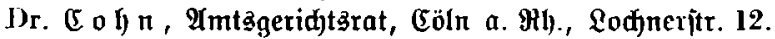

Dr. (E o h $n$, Beorg, Brojefior, Bürid $V$, (Earmenítr. 18.

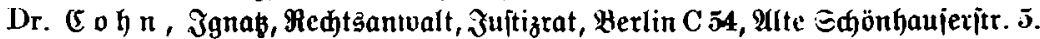

(io $\mathfrak{h} n$, Rouis, Rechtsantwalt, Motar, Jujtizrat, Berlin $N \overline{5} 4$, Brumenitr. 25.

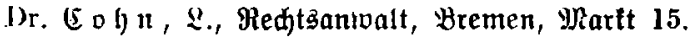

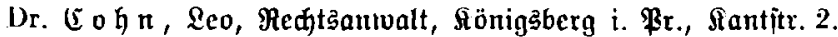

L゙ o $\mathfrak{h} n$, Souis, Juftizrat, Berlin W, Gruneivalditr. 91.

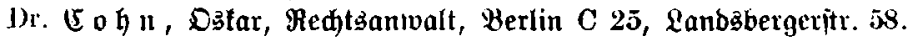

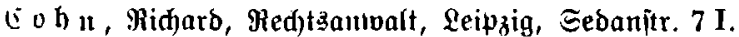

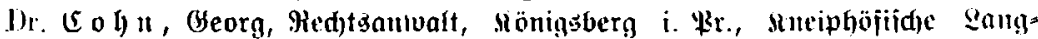
gafie 45.

Dr. $\mathfrak{C}_{\mathrm{oh}}$, Georg, Med)tanmualt, Eejiau, Gavalierftr. 8.

(i口 $\mathfrak{h} n$, Grorg, Med)tantwalt, Motar, Suftizrat, Iillit.

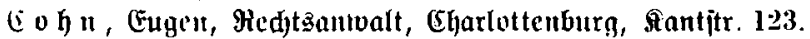

J)r. CE $\cup \mathfrak{G} \mathfrak{n} \mathfrak{b} \mathfrak{e} \mathfrak{g}$, Hedtsananualt, Graubenz.

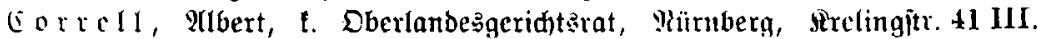

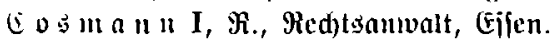

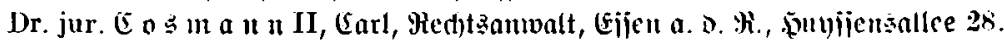

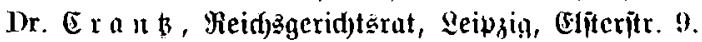

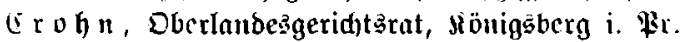

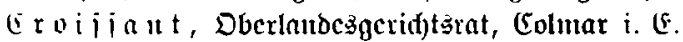

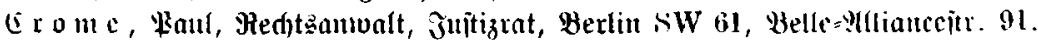

Dr. E r o ill e , Garl, Frojefior, Bonn, Siaijerftr. 61.

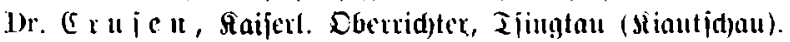

I a equlc, :Imtzridter, Mtehlauten.

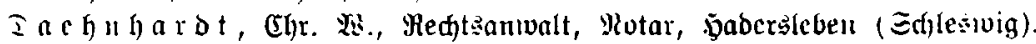

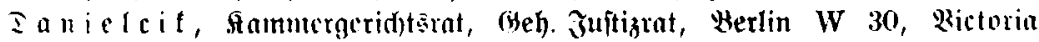
Souifeuplak I I.

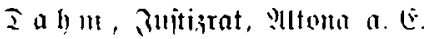




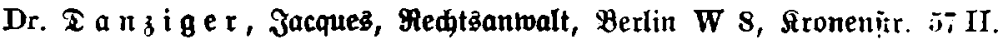

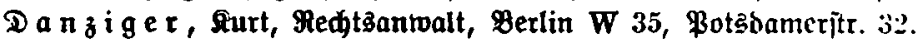

Da $\mathfrak{n}_{z}$ i g e $\mathrm{r}$, Mrax, Medtsanwalt, Berlin C 25, Dirđienitr. 22.

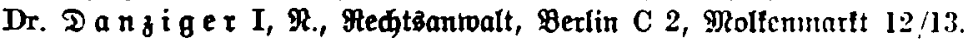

Da nz i ger, Medtzantwalt, Rotar, Brieg (Bez. Breallau).

Dr. D a $r$ m f a e $b$ t e $r$, Redtzantwalt, Mannheim.

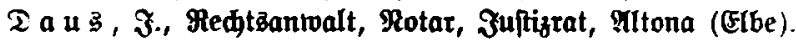

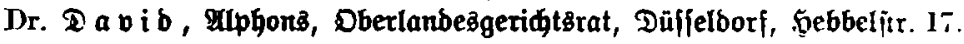

Da vi b , Gugo, Mectsanwalt, Frantenthal (Pialz).

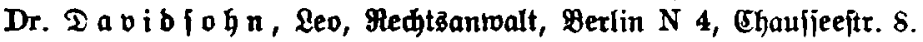

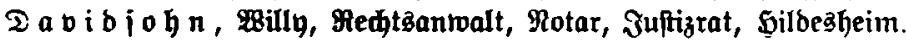

De b olv $\mathfrak{l}$, Rectzantwalt, Jultizrat, Rotar, Cottbus.

Dr. Dege $n$, Dberlanbeżgeriøtşrat, Dreäben, Dürerftr. $116 \mathrm{II}$.

Dr. Dehn , Dtto, Rehtsanwalt, Samburg.

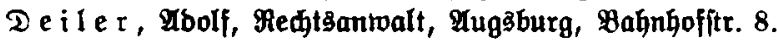

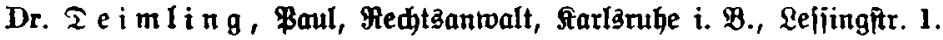

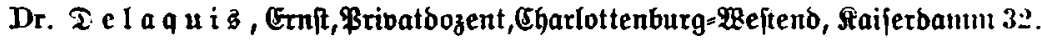

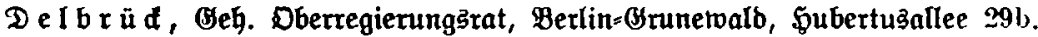

Dr. $\mathfrak{D}$ eli $\mathfrak{i} 3$, Rammergeridt

Dr. De nefe, Redtgantoalt, Böttingen.

Dr. De nnI er, Rgl. Rotar, Münden, Reuhaujeritr. 6 III.

Dr. Det mold , Beorg, Brofeffor, Böttingen.

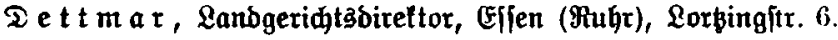

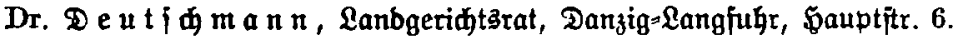

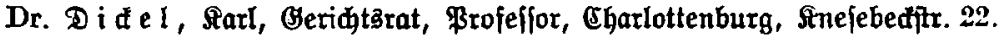

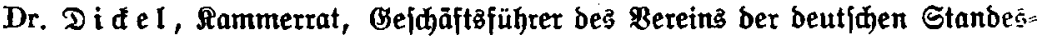
herren, 2 ernigerobe.

Dr. Di e $\mathbb{a} \mathfrak{m} \mathfrak{a} \mathfrak{n}, \mathfrak{B}$., Sujtizrat, Rechtsantualt, \$aunobci.

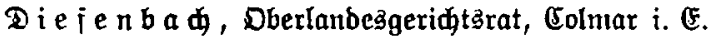

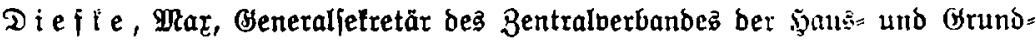
bejiser, Berlin W 15, Düfielborferjtr. 76.

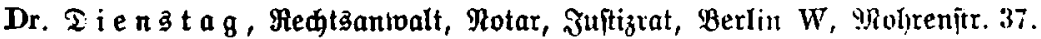

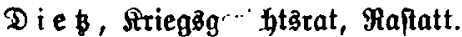

D i c b , $\mathfrak{B}$., Meidsantwalt, Leipzig, Saifer Milhelmitr. $: 2$.

Dr. T i e B, F., Redtanantwalt, Farlanube i. $\mathfrak{B}$.

Di e B, B., Redtsanmalt, Mtülbaufen i. (E., Mltfird)enftr. 33.

Die tri i, Redtsantwalt, Juptizrat, Frenzlau.

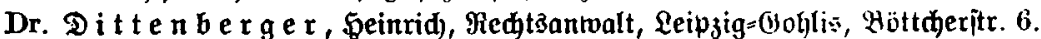

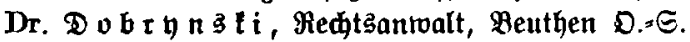

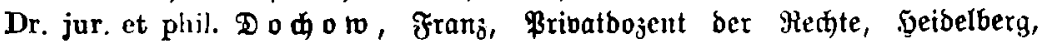

Biegelhaujer Lanbjtr. 59.

Dr. jur. To b n a, Alezanber Graf, Brofeffor, Sünigaberg i. Br.

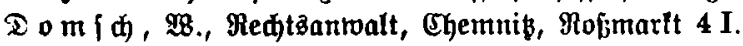

Toering, Lanbgeridłtapräfibent, Graubenj.

Doering , Ernft, Redtşantwalt, Snnbilus, Bittau, Lefiingitr.

Doe r mer, Medtżantoalt, Elberfelb.

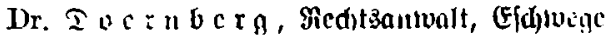




\section{XXIII}

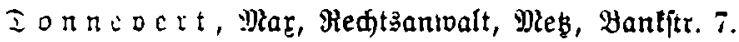

Dr. I o r a the 1 , Rehtsantoalt, Jujtizrat, Erejelb.

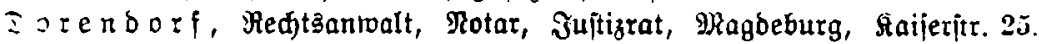

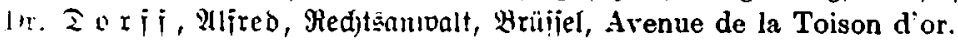

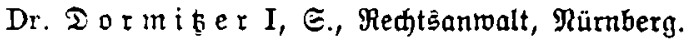

Dr. Tornc

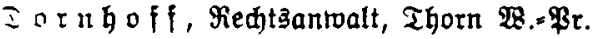

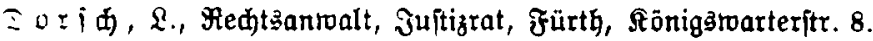

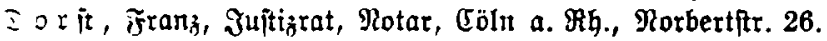

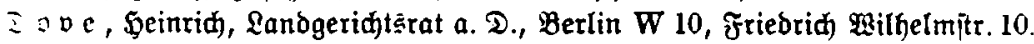

J). Iratben, Redtsunna!t, Jultizrat, Ereie!b.

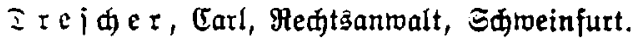

I c soner, Mar, Redtsantoalt, Liegniz.

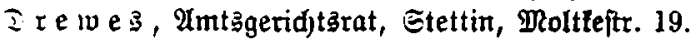

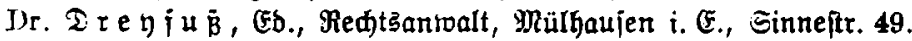

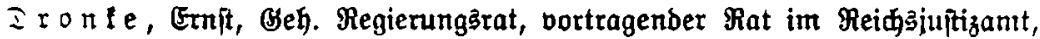
Berlin=Wilmerşbori, \$ialzburgeritr. 17.

Dr. : $\tau$ o it, Med)tzantoalt, Breşla V, Bartenitr. 47.

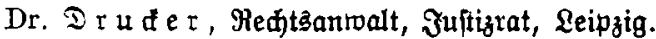

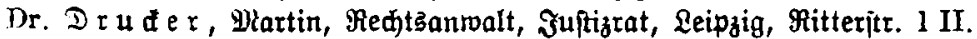

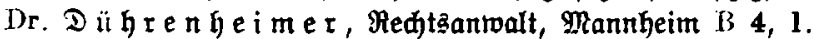

I ü mling, P., Rerhtsanmalt, Turlad.

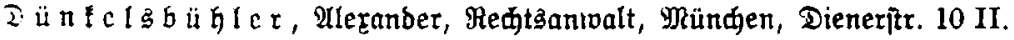

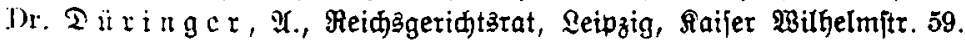

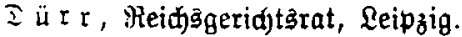

I ; $i$ a

Jr. (E bert, Senatäpräfibent am Stammergeridgt Berlin=Şöneberg.

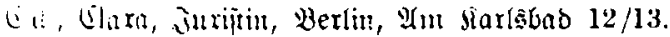

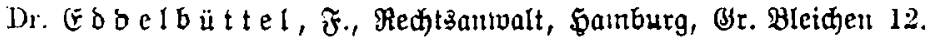

J). (E D c r, Carl, Keđțantbalt, Mannheim.

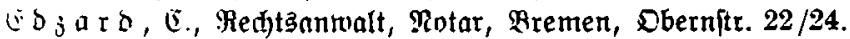

Dr. E $g$ e $r$, Redtizantwalt, Berlin W 8, Marfgrafenftr. 35.

Lig ger, Frofeljor, 3ürid V, Eprenzenbühl 20.

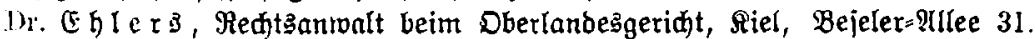

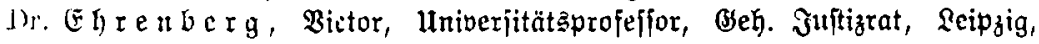

Bismarditr. $s$.

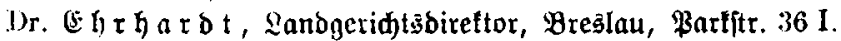

(E) $\mathfrak{r}$ I

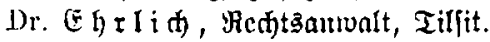

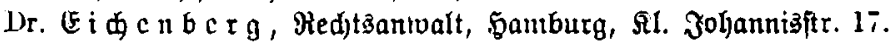

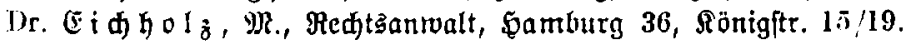

Dr. E i d I e $r$, Zanogerid)t3rat, Buben.

(E)

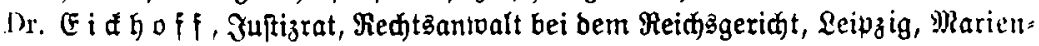

itrabe 11 .

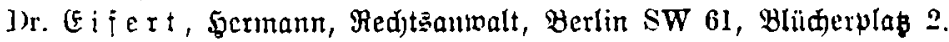

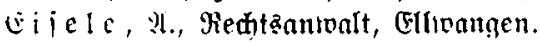




\section{$\mathrm{XXIV}$}

Dr. (E i j e m a $n \mathfrak{n}$, Mlar, Ređtşanivalt, Mürnberg.

E i f e $\mathfrak{n} \mathfrak{m} \mathfrak{n} \mathfrak{n}$, Jujtizrat, Berlin $\mathbf{4}_{4}$, Ehauffeeitr. 10.

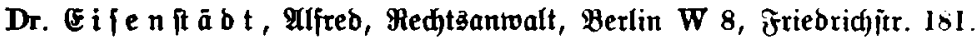

E i f e $n$ ft à b t, Redtsantoalt, Berlin SW 48, WBilhelmitr. 20.

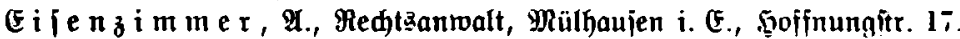

E If ner, Etabtredtätat, Baben=Baben.

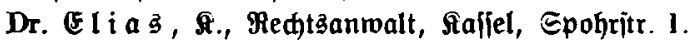

Dr. El te le 3 , Rejerenbar, Bojen, Maumanmitr. 10.

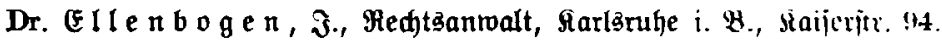

Dr. EII e $r$, Lanbgeridtşbirettor, Sarlşnuhe i. $\mathfrak{B}$.

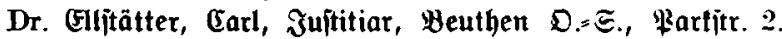

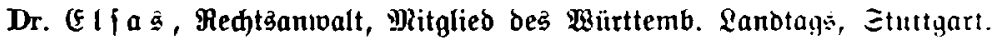

Dr. Eliäjier, Eenatspräijent, Etuttgart, Elgajtr. 6.

(EI $\bar{s} b$ a d), Redtżanivalt, Berlin W 64, Behrenitr. 48.

EI sa ner, Medtäantualt, Motar, Soiel $\Sigma=\Xi$.

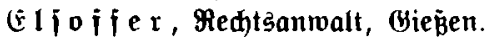

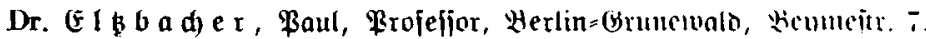

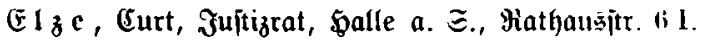

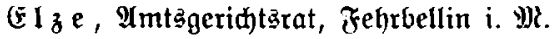

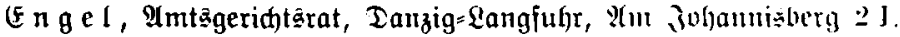

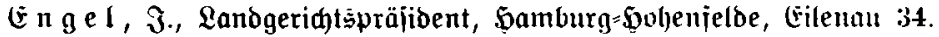

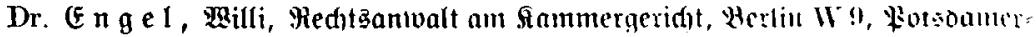
jtrá̃e 108.

Dr. E $\mathfrak{n} \mathfrak{g} \mathfrak{e} 1$, Dberlandes̄geridtstat, Bojen.

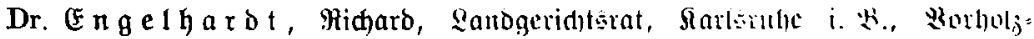
itrape 35.

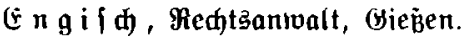

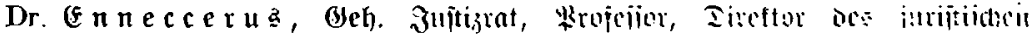
Eeminara, Marburg $i . \cdot \mathfrak{p}$.

Dr. Enneccerus, Geh. Jutizrat, Hrojejior, Marburg i.

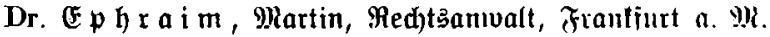

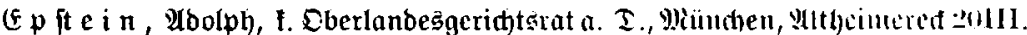

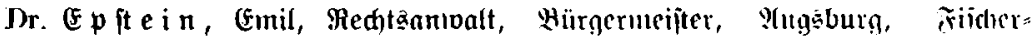
ftrabe B 239 II.

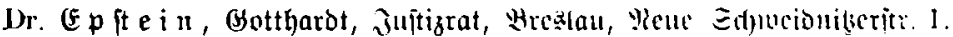

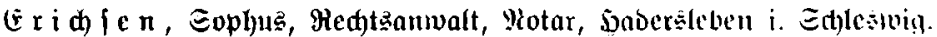

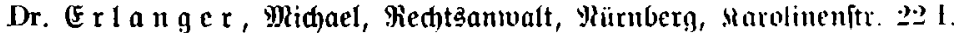

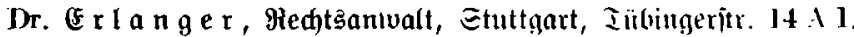

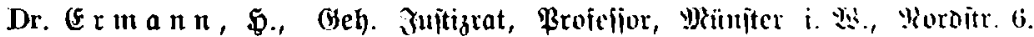

Dr. Erythropel, Geheimer Suftizrat, sammoner, (Hellertitx. 4 s.

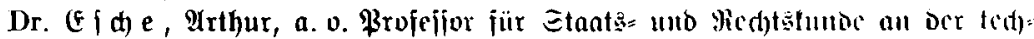

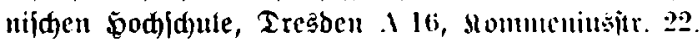

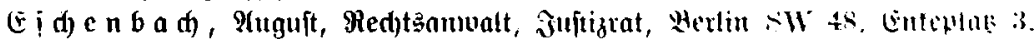

Dr. E i f e I b or I, Sarl, Bibliothetar, Darmitabt, Martinsitr. 4:.

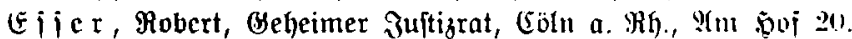

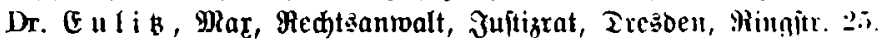

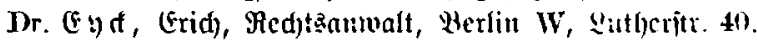




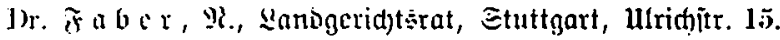

î a bia

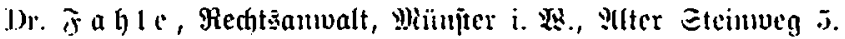

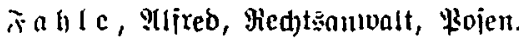

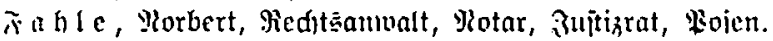

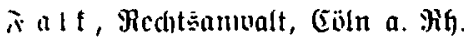

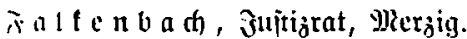

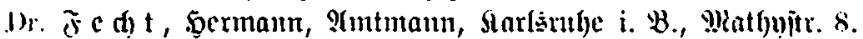

示

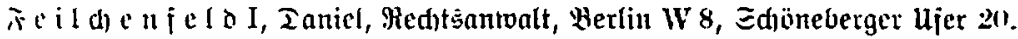

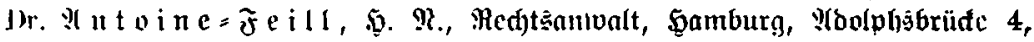
Hörienfaus.

1)r. E e i j

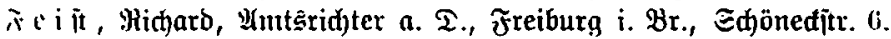

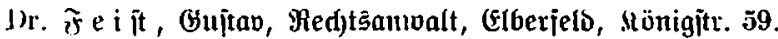

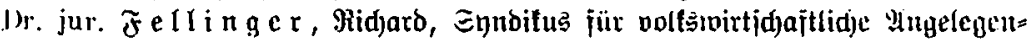

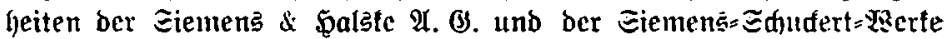

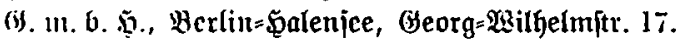

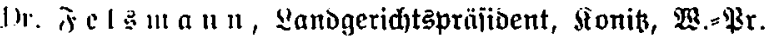

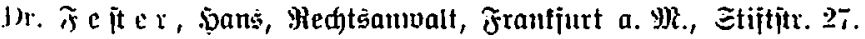

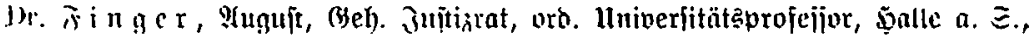
Meiduarotfitr. 2.

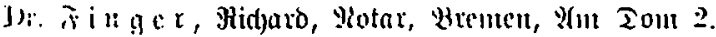

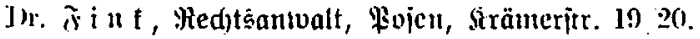

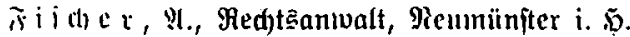

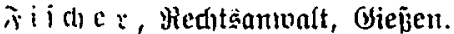

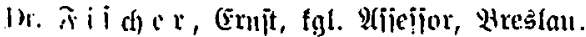

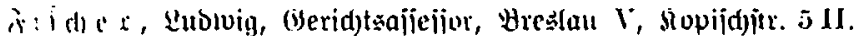

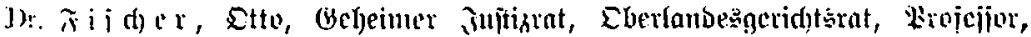
Hristau, Mlonthauptitr. 3.

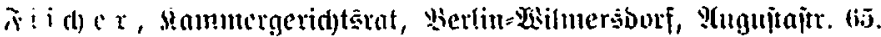

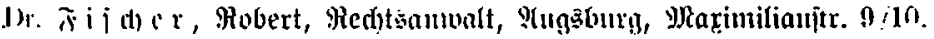

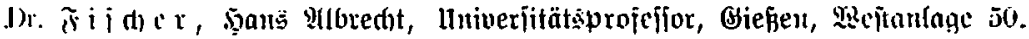

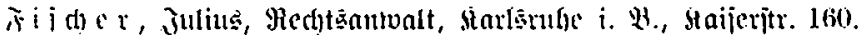

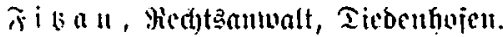

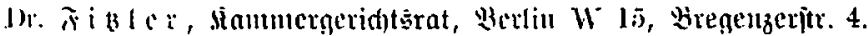

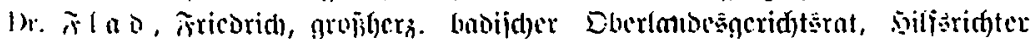

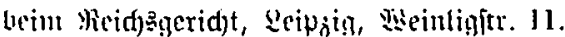

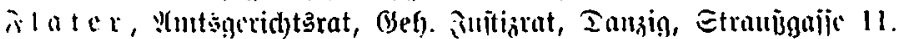

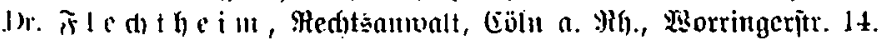

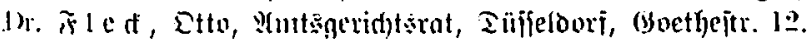

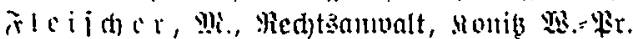

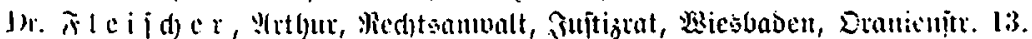

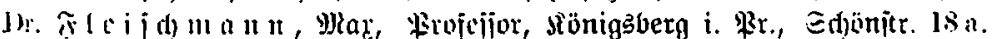

Fl itis, Medtsambalt, Motar, iuftizrat, Fraujtabt.

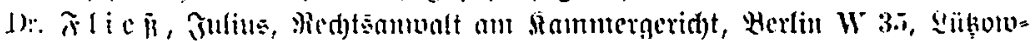
itraije $4 !$ ! 


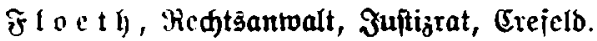

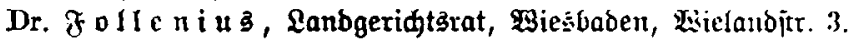

Dr. F o I 1 c r, Medttzantwalt, Dortmund, Ratieritr. 1.

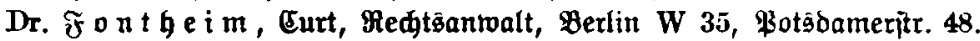

Dr. Fo n the i m, Georg, Redtsantoalt, Berlin W 8, Mohrenítr. 41.

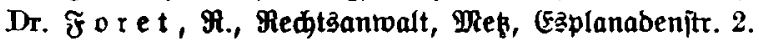

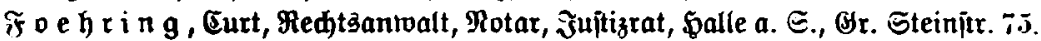

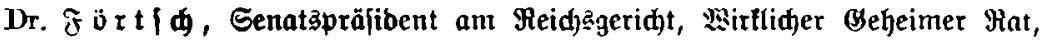

Seipzig, Mlbertitr. 32.

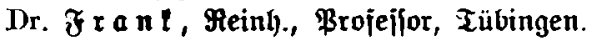

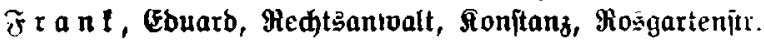

Dr. \& $\mathbf{r}$ a n!, \&ubwig, Rects̄anwalt, Mannhein B 1, 11.

Fra $n$ de, Friz, Redtsantwalt, Danzig, Sundegajie 94.

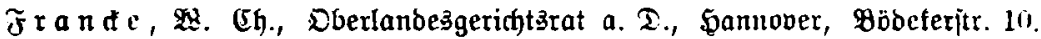

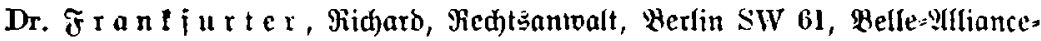

Plab 4.

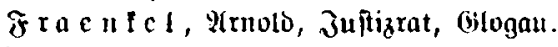

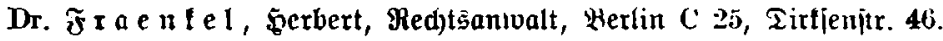

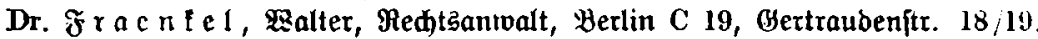

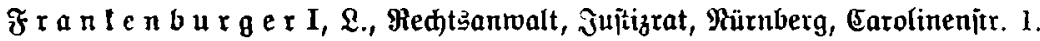

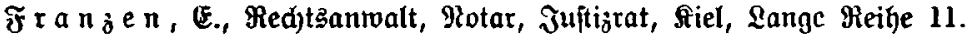

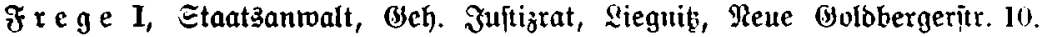

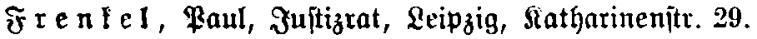

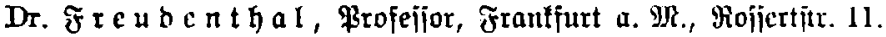

F $r \in \mathfrak{n} b$, Rubroig, Rechtsantwalt, Mannheim.

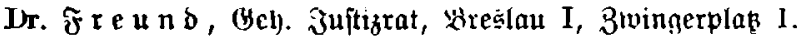

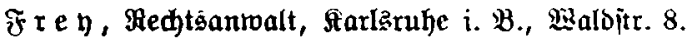

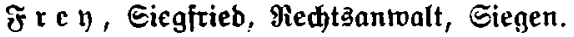

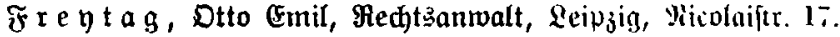

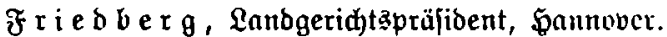

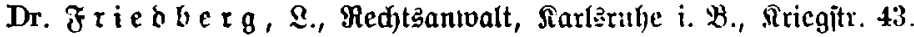

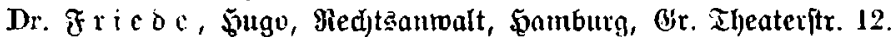

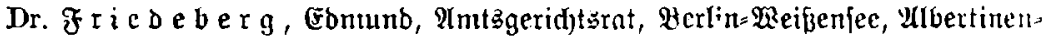
irrab́e ly.

Dr. F г i e b e m a n, Gomuno, Jujtizrat, Berlin W 35, Starlsbad 4a.

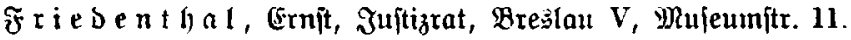

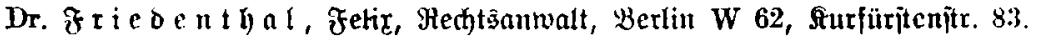

Fri e d a jitrafie 7 .

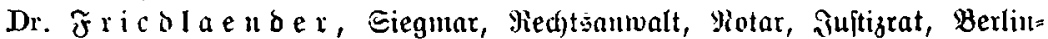
(Sharlottenburg, Meue fantitr. 1.

Dr. Fric ol a e ner, Georg, Bantoirefter, Jutiziat, Wherlin W 8, Sillhelm= itrufe $i 1$.

Frieblat $n$ ber, Redtandoalt, Bromberg.

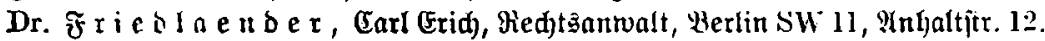

Frie ol a e nder, Fr., Juftizrat, Berlin W 35, \&üboloftr. 46.

Fr i e l a e n 


\section{XXVII}

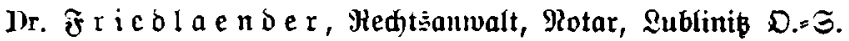

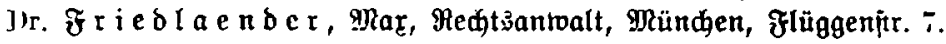

J). $\mathfrak{F} \mathbf{r}$ if $\mathfrak{d} \mathfrak{i} \mathfrak{b} \in \mathfrak{n}$, Frib, Geheimer Juftizrat, Frantfurt $\mathfrak{a} . \mathbb{P}$.

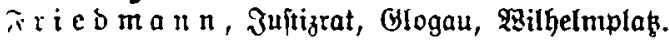

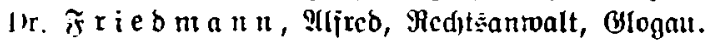

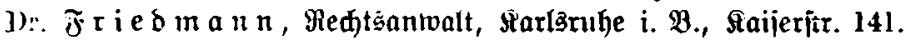

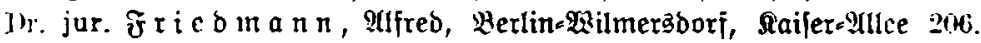

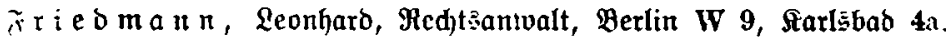

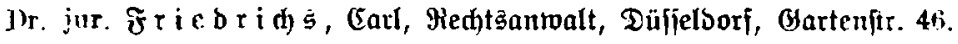

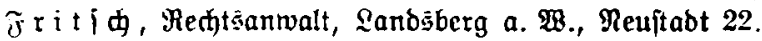

])r. Fr i b c , Zanoridter, Mauntburg a. E., Bartitr. 18.

సx i $\mathrm{c}$, Beh. Eberjuitigrat, Berlin W 62, Wormierftr. 11.

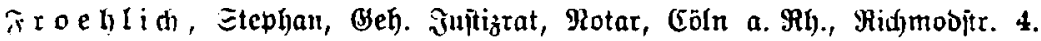

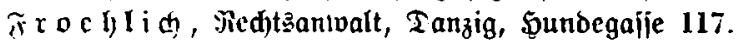

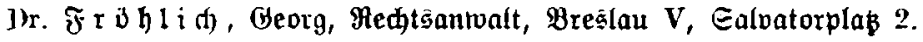

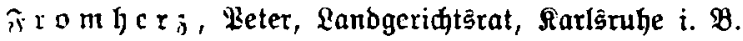

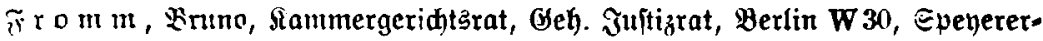
itraije 1.

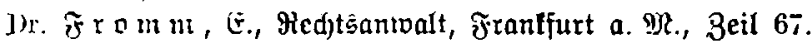

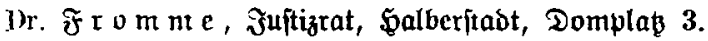

Dr. Fro m m hold beorg, Brojeifor, Beh. Jujtizrat, Greifsivald.

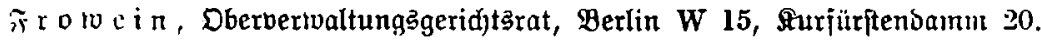

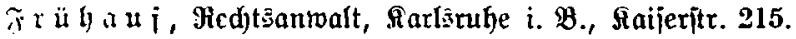

Dr. ₹ॄ 山

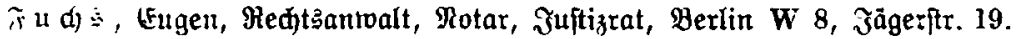

i) $u$ d) I, Mkaz, צuftizat, Berlin W 9, Botabamerftr. 13.

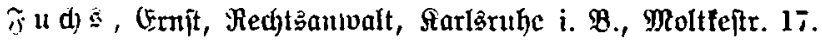

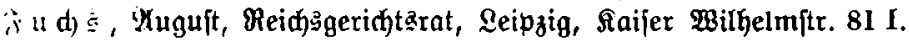

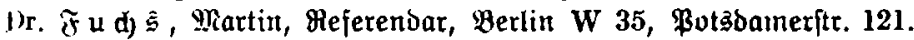

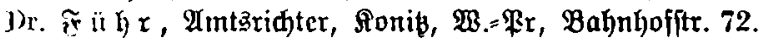

if ul h ne, Jofef, Juftizrat, Sïnigaberg i. Br.

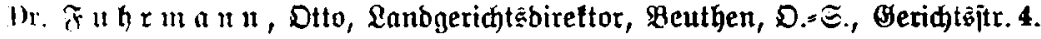

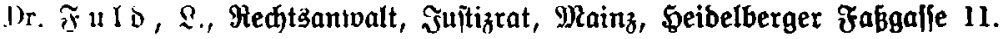

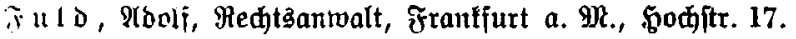

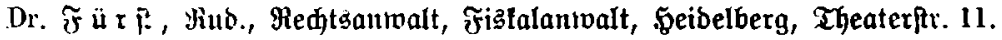

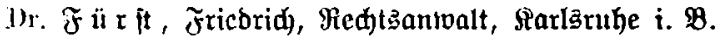

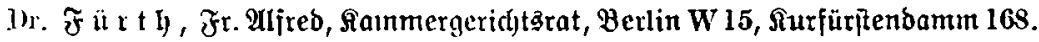

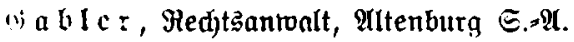

(i) a c bulth, Ranogerid)terat, Berlin NW 23, Glaubiusitr. 13.

Dr. (ii a i s id), Reditsanwalt, Motar, Inftizrat, Chemnik, Theateritr. 84.

ir. Gi a lla $\mathrm{n} D$, Redtzmontualt, Boien.

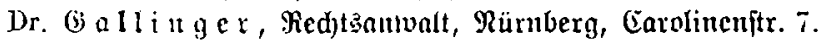

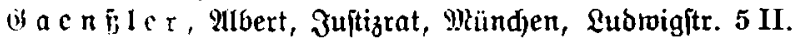

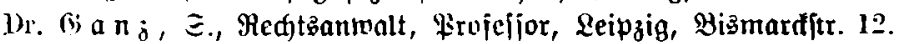

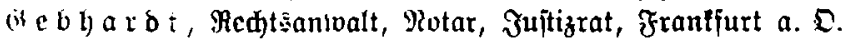

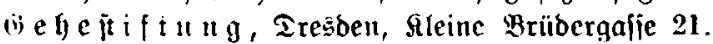

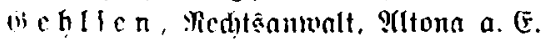




\section{XXVIII}

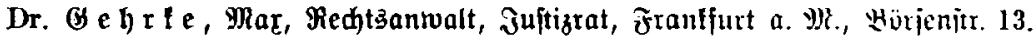

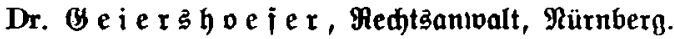

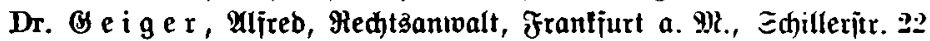

Dr. Bs e i ger, Berthold, Medtsanvalt, Motar, (Geb). iluitizrat, Frantiurt a. \$ll, Edillerftr. 22.

Dr. Gs e iger, Medtzanwalt, Sandöberg a. Ma., Midhtitr. il.

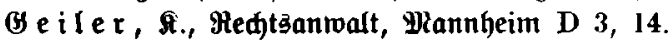

(H) $i \bar{B} l e r$, Oberiuftizrat, Freiberg $i . \Xi$.

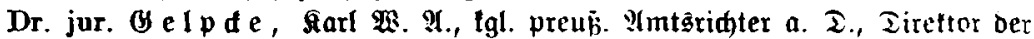
Sypothefenbant in Samburg, Berlin NW 23, Bridtenallec 4.

G e n t i I, 3., Medtsaniraalt, Mannheim.

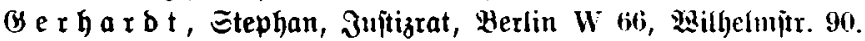

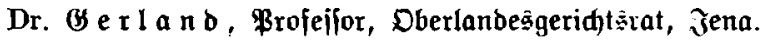

Dr. Gernsheim, Lanbridter, Djtrowo i. Hojen, Sütolvitr. 7 .

(G) $\mathrm{e}$ id $\mathrm{t} c$, Jujtizrat, Berlin $W 8$, Jägerftr. 19.

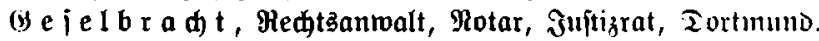

(i) c if e le, D., Redtsanmalt, Memmingen.

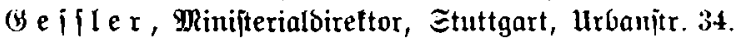

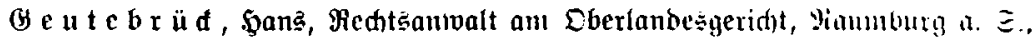

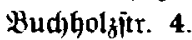

Dr. v. B i e r fe, Dtto, Geb. Jutitizat, Proi., Chartottenburg, Eumeritr. 1:2.

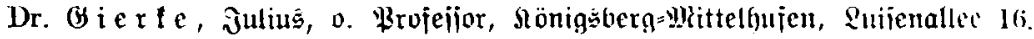
(i) i e i e d e Jujtizrat, Magbeburg, Breiterveg 230.

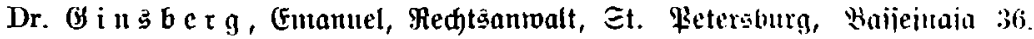
(i) $\mathfrak{l} \mathfrak{a} \mathbf{m} \mathfrak{a}$ d) $\mathfrak{e} r, \mathfrak{E}$. , Motar, Mombad i. Sothringen.

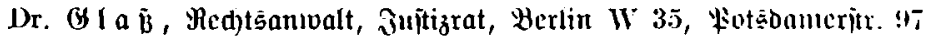

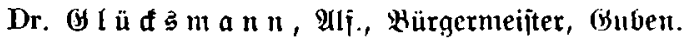

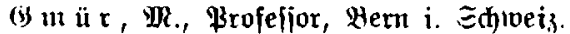

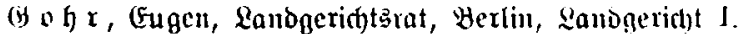

(i) ol b be rg, Med)tiantualt, Ylotar, Zujtizrat, Marburg a. D. Salm.

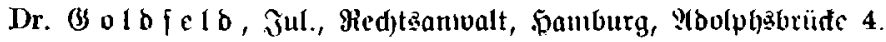

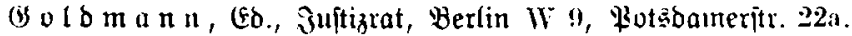

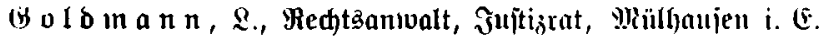

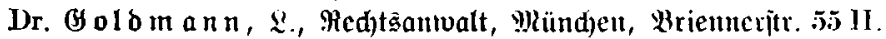

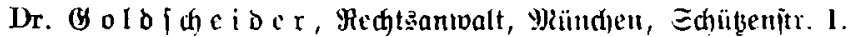

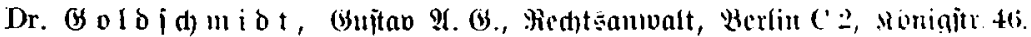

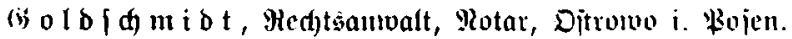

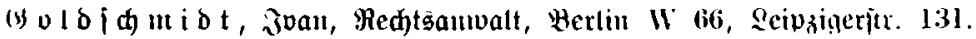

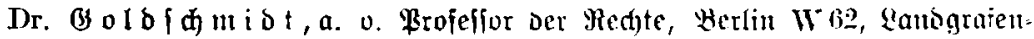
iträ̉e 9.

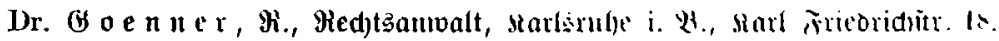

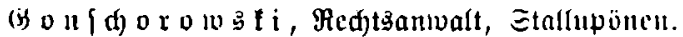

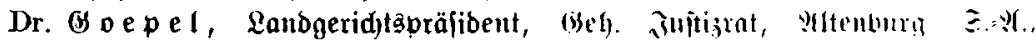
Langengalie 10.

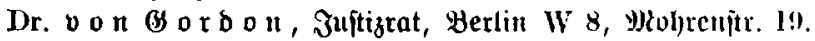

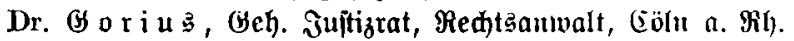

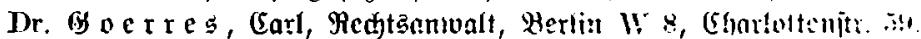




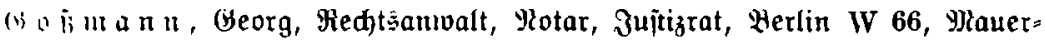
itrape $66 / 67$.

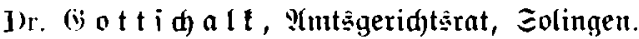

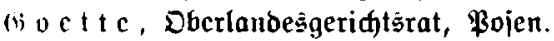

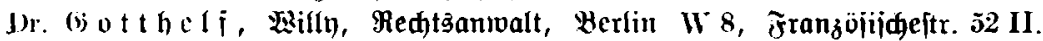

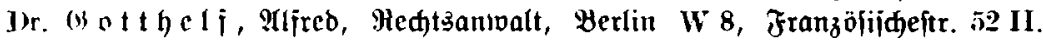

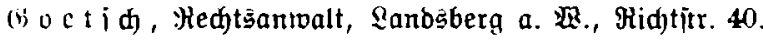

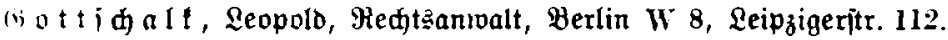

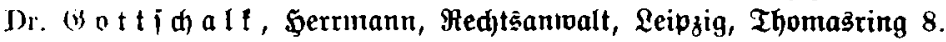

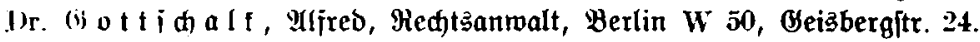

Jr. (i) r a o e $\mathrm{n}$ w i b., Projeijor, feeibelberg, Meuent). \{anbitr. $8 \mathrm{I}$.

Dr. ํ о

Dr. (i) r a $j \bar{i}$, Red)tsanvalt, Freiburg i. Br., Eijenbahnjtr. 2.

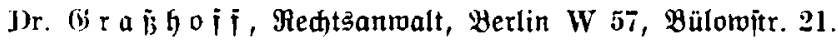

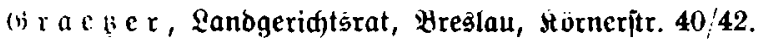

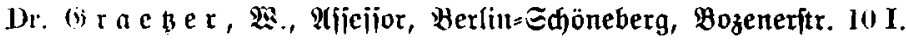

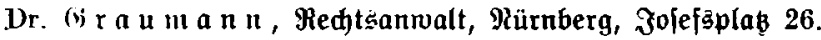

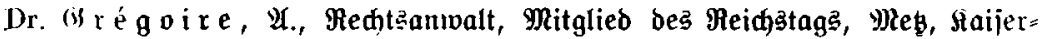
xillhelın= Hing.

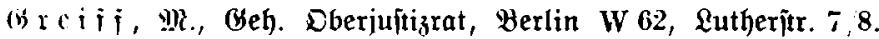

Dr. jur. Of reil, Eubivig, Berlin=steglib, Flenğburgeritr. 6 .

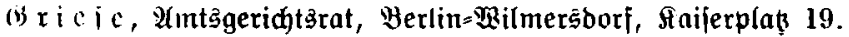

if $r$ i e $i \in c r$, Jutitizrat, Eolberg.

i) $\mathrm{r}$ i $\mathrm{ll}$, Zandgeridht

if $x$ a b c, $\mathfrak{S}$., Medtsanivalt, Motar, Jujtizrat, Calbe a. 5 .

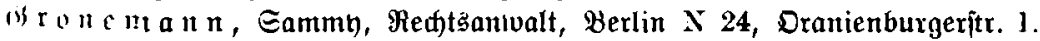

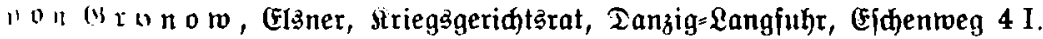

(i) i u :

(1)r. (si r o te, G., Fidfter, Bremen, Mrathilbenitr. 27.

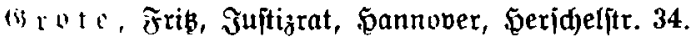

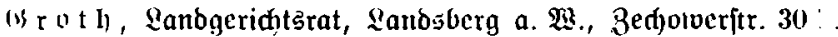

Dr. (ii r uc $\mathfrak{b} \in \mathfrak{r}$, Ertv., Brojelivor, Münd)en, Bijelajtr. 5.

(is $r$ u $m$ b a d e $r$, Otto, Redtsantivalt, Starlstuhe i. $B$.

" o

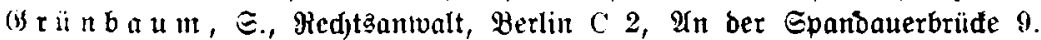

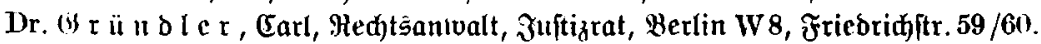

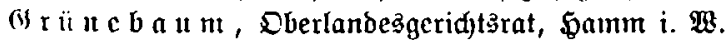

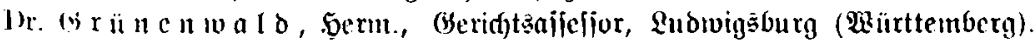

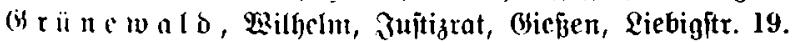

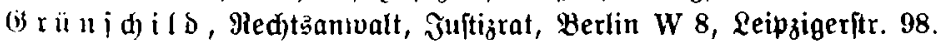

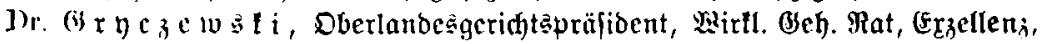
Piojen.

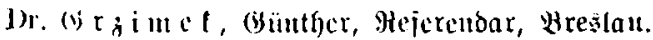

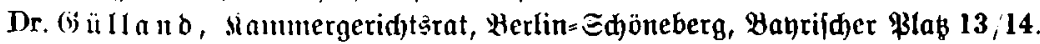

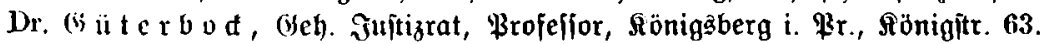

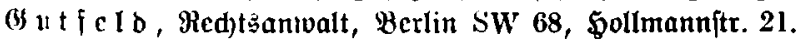

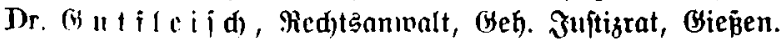


Dr. B u t herz, Rebtzanwalt, Beuthen $\Sigma=\Xi$, Bahnhofitr. 3.

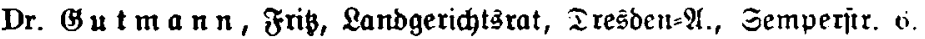

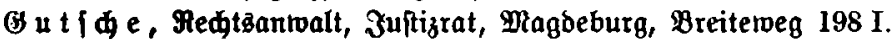

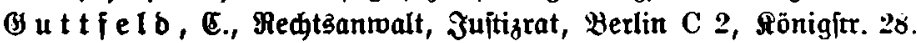

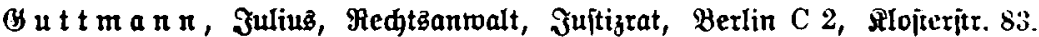

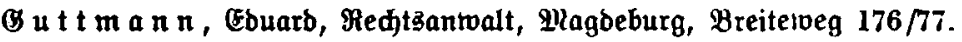

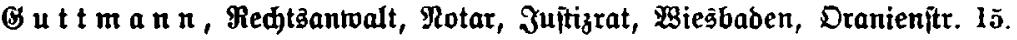

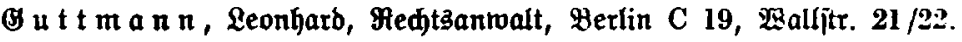

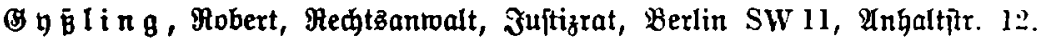

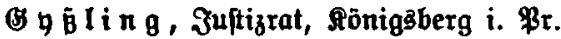

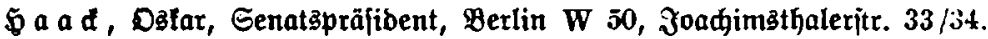

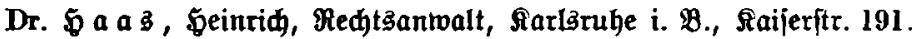

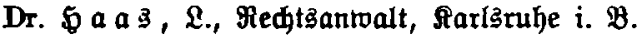

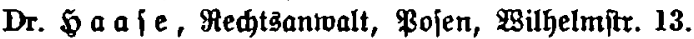

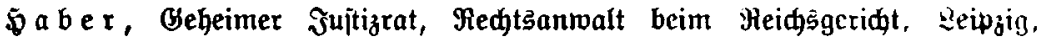
Sđdroägriðenftr. 13.

Dr. 5 a b e $\mathbf{l}$ ing , Ss., Redtzantwalt, Mainz, Staijeritr. 7 .

Dr.

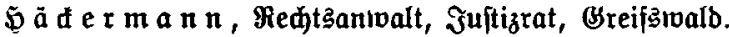

$\mathfrak{G}$ a o r $a$, Urtbur, Ređts̄antralt, Berlin W 8, Ranonieritr. 45.

Dr. $\$$ a f $t$ e $r$, E., Brofeffor, Fildberg $\mathfrak{b}$. 3ürid).

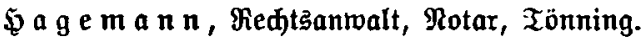

¿ a g e $n$, Dtto, Fammergeridtarat, Berlin W 30, \$aberlandïtr. 5 .

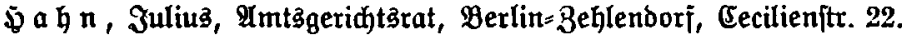

Sa hn, Franz, Redtsannalt, Berlin C 2, Spanbauerbrüđe 2.

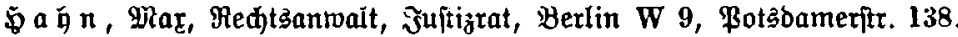

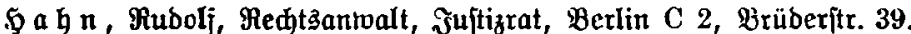

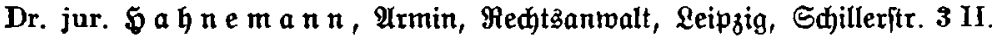

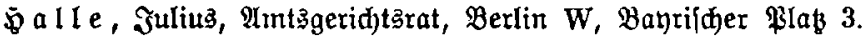

Dr. Salle, Medbtsanmalt, Notar, Suftizrat, Berlin W 8, Rronenīt. 56.

Sa ll e $\operatorname{s} \mathfrak{l}$ e $b$ e $n$, Redtzanwalt, Berlin C 2, Epandauerftr. 63 a.

Da II ro a $s$, Jujtizrat, Darmftabt.

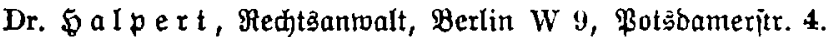

Sa m b u r ge r, (Earl, Rechtsantoalt, Jujtizrat, Berlin W 35, Botşbanterftr. 3I.

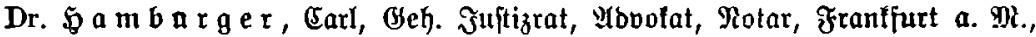
Corneliuştr. 8.

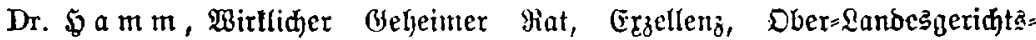
präjibent, Bonn, Suantiustr. 8.

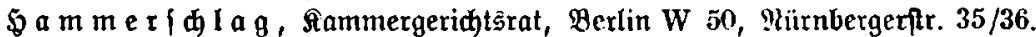

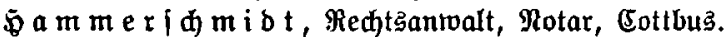

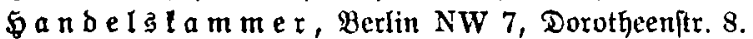

$\bar{\Psi} \mathfrak{a} \mathfrak{n} \mathfrak{e}, \mathfrak{R e d}$ tşanwalt, bcrlin W 9, (Fidhornitr. 1 .

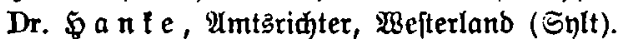

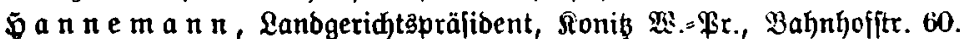

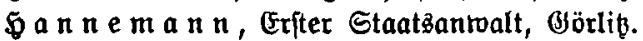

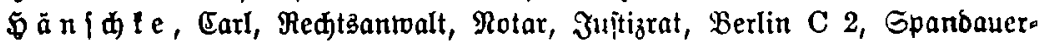
prafe $47 / 48$. 


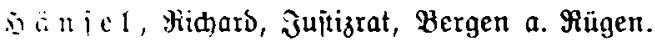

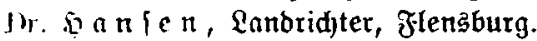

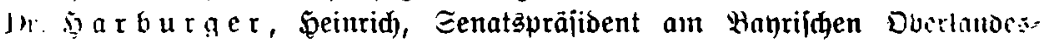
gerid), ltniberiitützprojeijor, Miünden, Garlitr. 21 .

Dr. Far beland, Hidter, Hremen, Moonitr. 79.

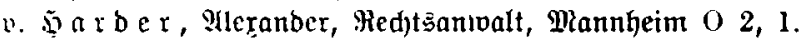

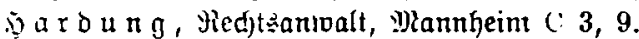

Dr. Garmening, Ermit, Redjtäantwalt am Eberlandeşgeridgt, Juịtiatat, 3ena.

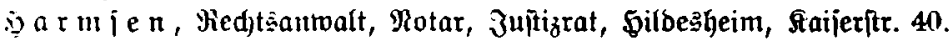

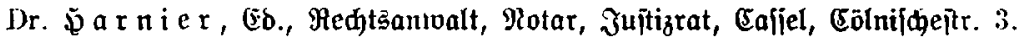

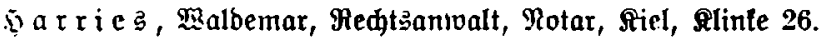

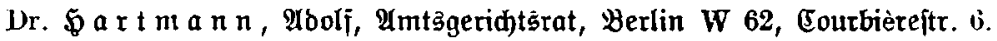

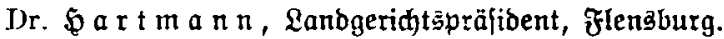

si a $\mathrm{t}$ m a $n$, Seo, Redhtsantwalt, Mülhaujen i. 5 .

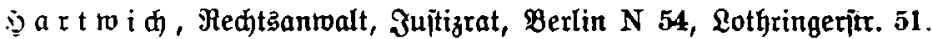

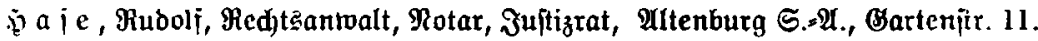

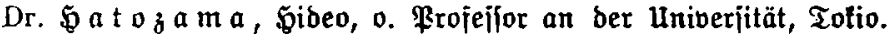

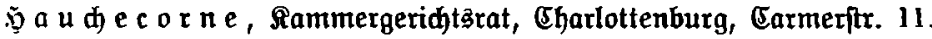

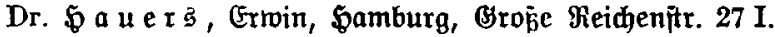

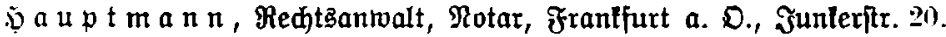

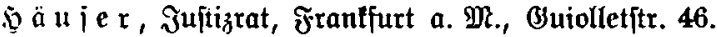

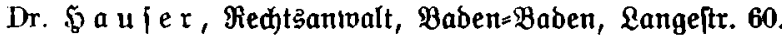

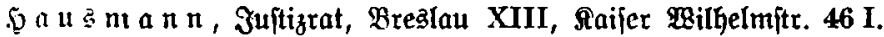

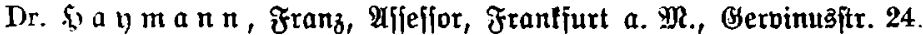

ij a i) $\mathfrak{n}$, Julius, Redtsantwalt, Berlin W 64, Behrenitr. 17.

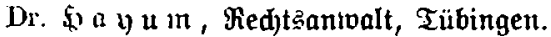

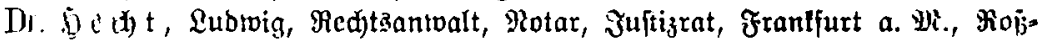
martt 18.

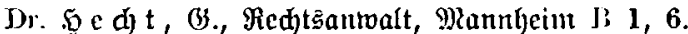

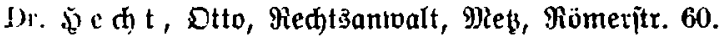

Dr. Sed, Frojeffor, Tübingen, Ređarhalbe 68.

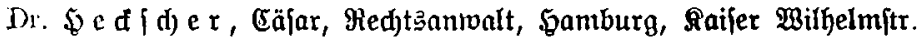

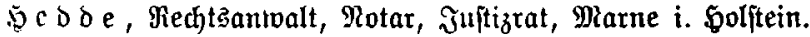

.

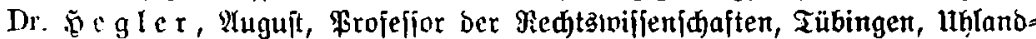
itrafie 2.

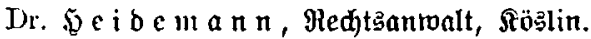

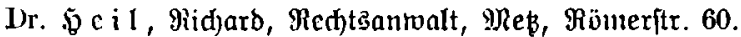

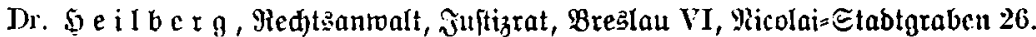

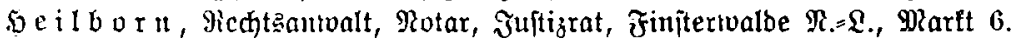

joc il b r u

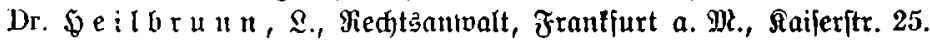

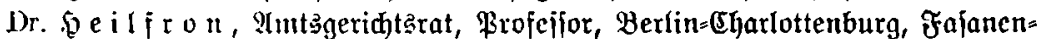

fientic 6.

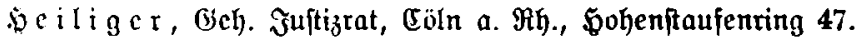

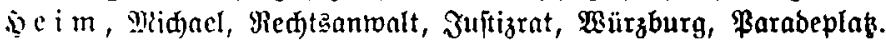




\section{Xxili}

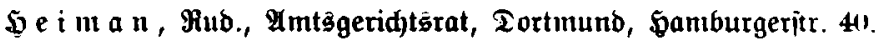

Dr. \$c i m a $\mathfrak{n}$, Jacob, Redt

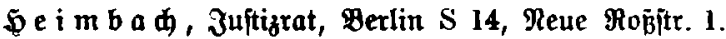

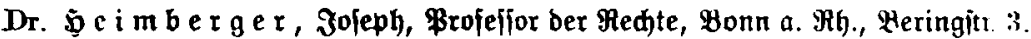

Dr. Şc ine ma nn, Medtąantwalt, Berlin W 8, Sronenjitr. 8/9.

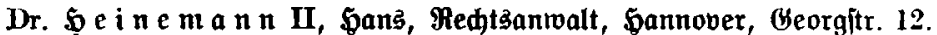

Dr. $\mathfrak{B}$ e i ne ma $n$, Jujtizrat, פRagbeburg, Saijeritr. 34.

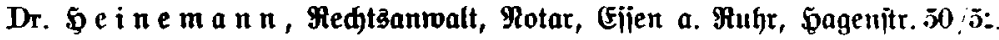

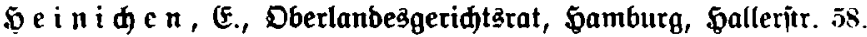

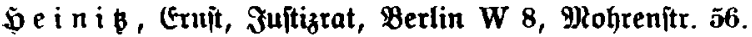

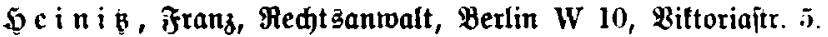

Dr. Sc in 1 , Baul, Mboolat, MRelibe (Iefijin), Billa Jibora.

Dr. Sc inrici, Lanbridter, Berlin=Ridhterfelde, Dratejtr. 43.

Dr. Fe in $a \mathfrak{h}$ e i un er, Carl, Brofeffor an ber univerjität, Seibelbern

Dr. Ge ije, Curt, Medhtzantoalt, Tanzig, Sundegajfe 20.

Se i ft c r be r g f II, Ređtzanibalt, Freiberg i. $\Xi .$, Dbermarft $\$ \mathrm{~J}$.

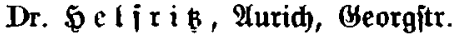

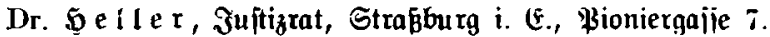

Dr. Sge ll m a n n, Friebr., Projelior, Münden, Babeläbergeritr. 1 a.

Dr. SE $\mathfrak{b} \mathfrak{c}$ un b b, 3uftizrat, Lanbjberg a. $\mathfrak{x}$.

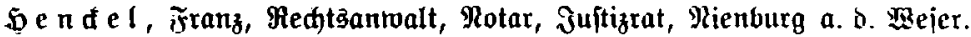

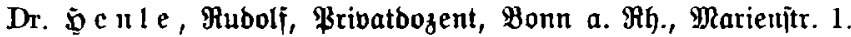

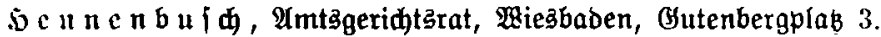

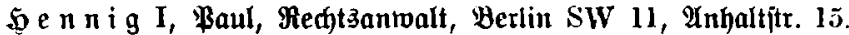

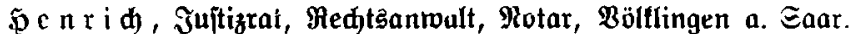

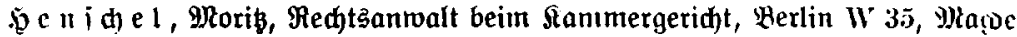
burgeritr. 22.

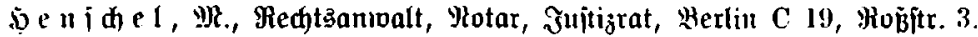

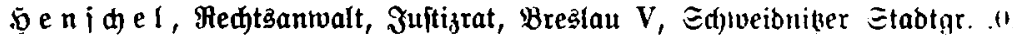

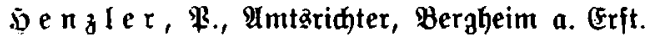

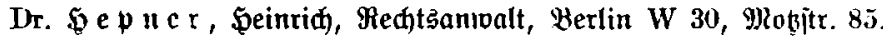

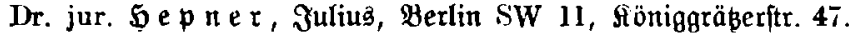

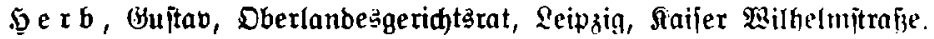

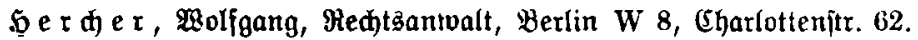

$\mathfrak{S} \in \mathfrak{x} \mathfrak{a} \mathfrak{a} \mathfrak{s}$, Eugen, Redtzantualt und tgl. Motar, Gillesheint a. Eijel

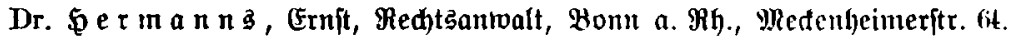

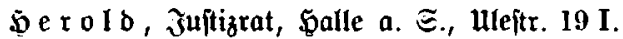

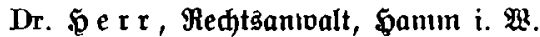

Serrenboerffer, Juftizrat, Elvinemünde.

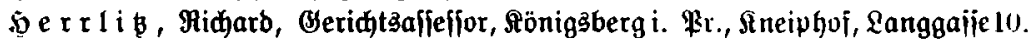

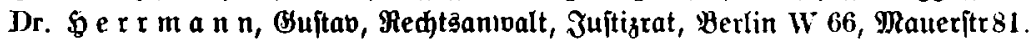

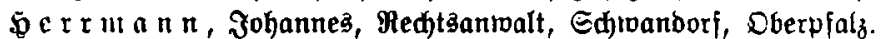

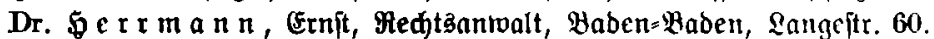

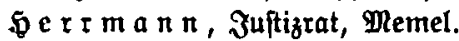

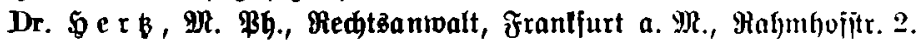

Dr. \$ूer , B., Senator, \$amburg, Magbalenenitr, 3. 


\section{XXXIII}

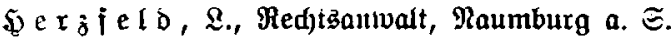

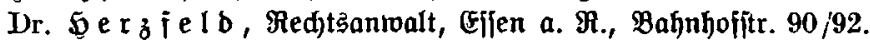

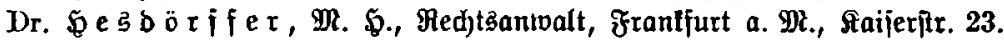

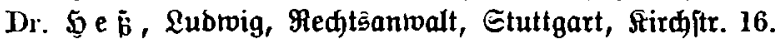

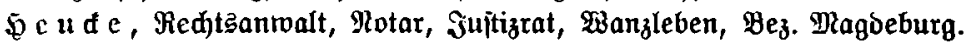

va n ber $\mathfrak{s e y b e , ~ F r . , ~ \Re e d t s a n t w a l t , ~ \Re o t a r , ~ J u f t i j r a t , ~ \Re u ̈ b e ⿰ 弓 ̆ e i m ~ a . ~ \Re h . ~}$

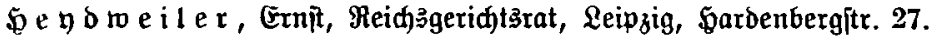

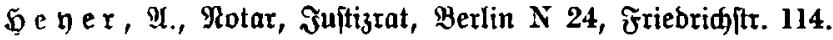

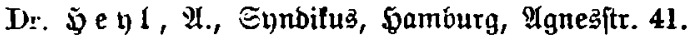

$\mathfrak{F} \in \mathfrak{y} \mathfrak{m} \mathfrak{a} \mathfrak{n}$, Sulius, Ğeheimer Suftizrat, IItona a. (E.

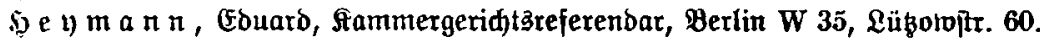

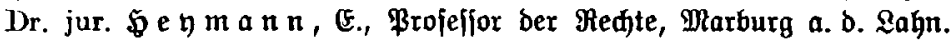

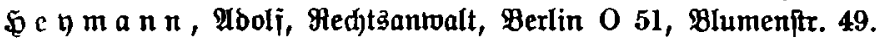

Dr. Sen m a n $\mathfrak{n}$, C̈buaro, Redtananwalt, Danzig, Jopengaife 11.

Dr. $\mathfrak{S} \mathfrak{y} \mathfrak{m} \mathfrak{a} \mathfrak{n} \mathfrak{n}$, S., Red)tzanwalt,

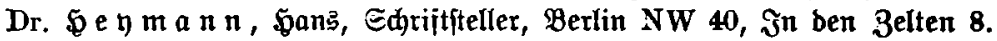

$\mathfrak{Q} \in \mathfrak{y} \mathfrak{m} \mathfrak{a} n \mathfrak{n}$, Redtsantualt, Bromberg, Burgitr. 22.

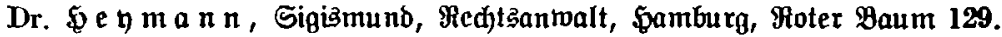

Fe n $n$, Georg, Redtsantwalt, Feitenberg b. Breslau.

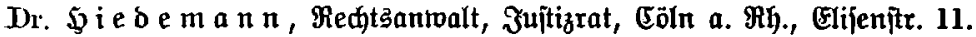

$\mathfrak{S i l}$ be brand, $\mathfrak{B}$.

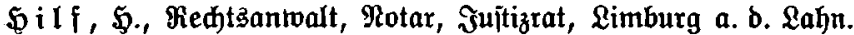

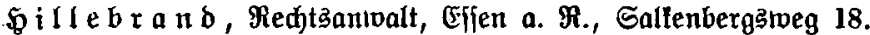

Dr. Gilpert, Juftizrat, Sigl. Kobofat, Rürnberg, \$auptmartt 11 I.

Dr. Gilje, Benno, Freisgerid)ts̄rat, Berlin SW 11, Röniggräßerftr. 61.

Dr. Silfe, Carl, Ennditus, Brojeffor, Berlin SW 11, Rüniggräłeritr. 61.

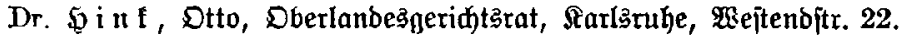

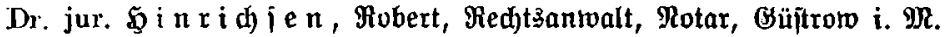

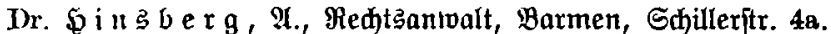

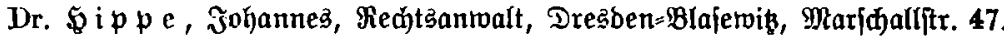

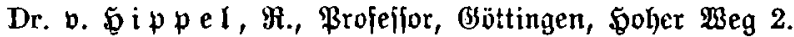

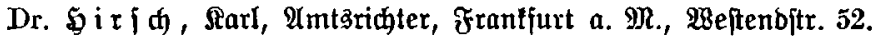

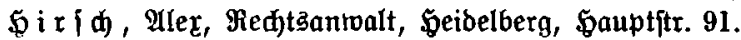

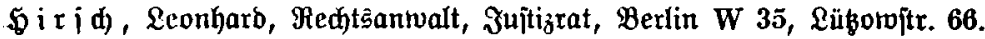

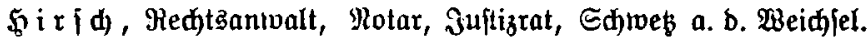

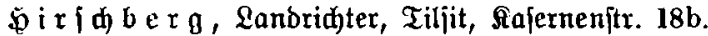

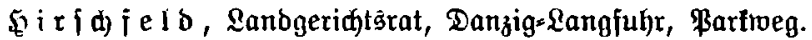

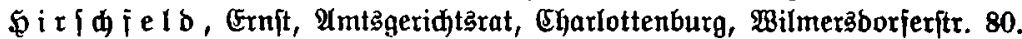

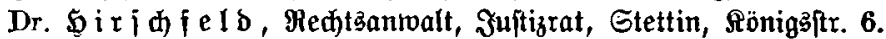

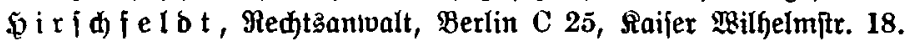

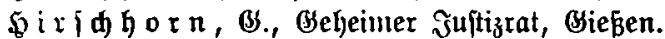

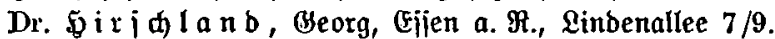

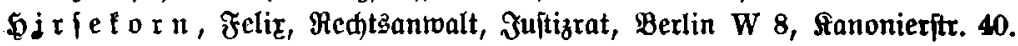

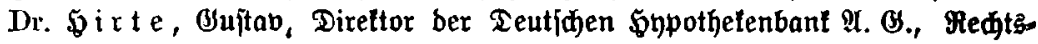
antwalt, Berlin $\times 2$, Dorotheenftr. 54 .

Dr. $\mathfrak{B} \circ b$ e, Berjidhenungşbireltor, Berlin W 50, Sulmbadieritr. 14.

fo bel ma $\mathfrak{n}$, Lanbgeridtabireftor, Bremen, Dobben 106. 
Dr. 5 o d f

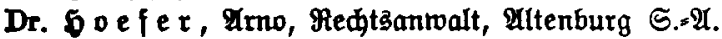

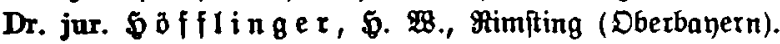

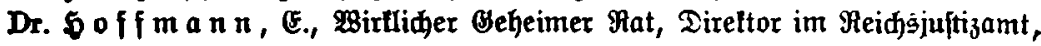
Erzellenz, Berlin W 62, Eourbièreitr. 10.

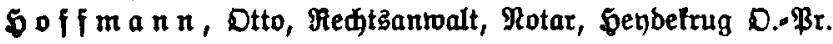

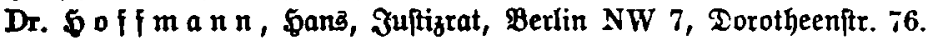

Dr. \$off ma n n, Max, Redtzanivalt, Rotar, Juftizrat, Berlin NW 7, Tauberitx. 33.

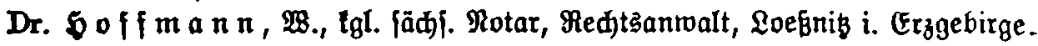

\$o ff $m$ a $\mathfrak{n} n$, Lanbgeriđtšat, Breslau IX, Sebroigitr. 38.

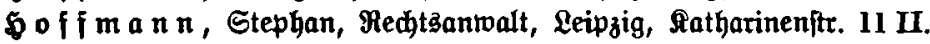

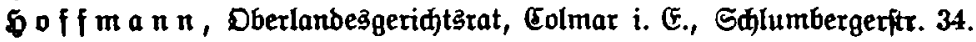

b of ff a e $b t, 28$., Medtsantwalt, 3uftizrat, Berlin SW 68, Bimmeritr. 94.

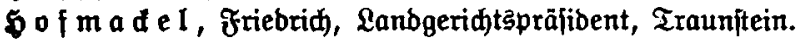

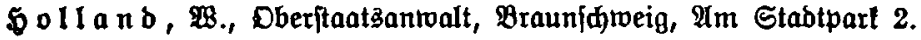

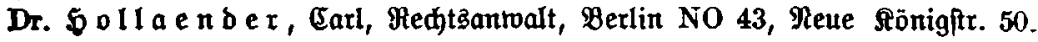

Dr. \$oll a e n b e r, Ulbert, Redtzantwalt, \$amburg, (Fr. Bleiden 23.

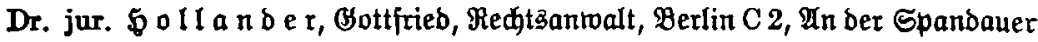
Brüđe 8.

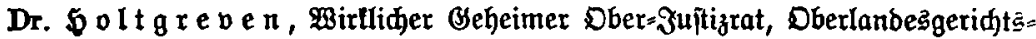
prälibent, Đamm i. $\mathfrak{x}$.

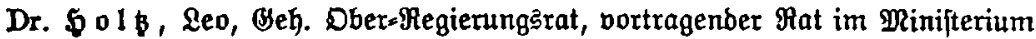
für Lanbwirt|đaft, Domänen unb Forften, Eharlottenburg, Berliner: Prápe $58 \mathrm{II}$.

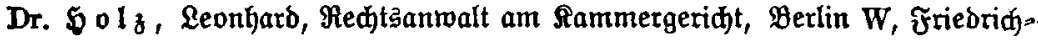
frape 14.

Dr. $5 \circ I_{z}$, Ferbinand, Red)täantoalt, Mannheim $M 2,9$.

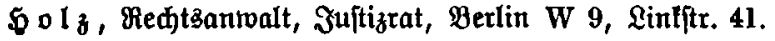

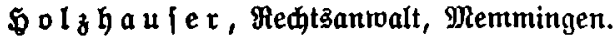

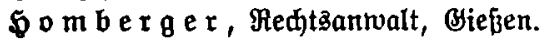

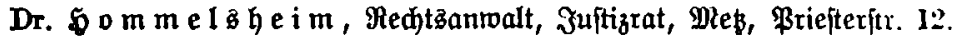

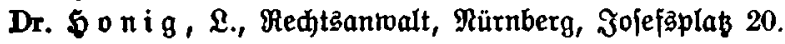

Soni 8 , צultizrat, Gnefen.

Dr. So e ni ве r, $\$$., Privatbozent, Freiburg i. B., Fabrifitr. 29.

Dr. Soeni ger, Sanogeridtärat, Sarlsrube i. B., Bismarđftr. 53.

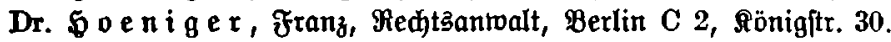

Dr. $\mathfrak{b}$ oepf ner, Brofeffor, Böttingen, Gerzberger Ehauffee 46.

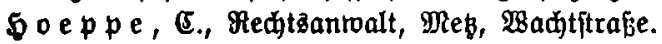

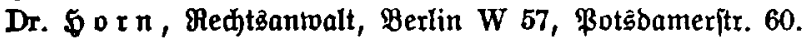

Dr. $\mathfrak{S}$ or $\mathfrak{n}$, Ređtsantwalt, Rotar, Bürgermeifter, Molbegl i. Medt.

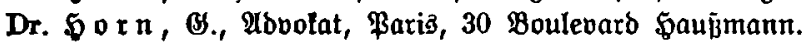

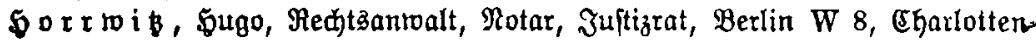
ftrá̧e $48 \mathrm{II}$.

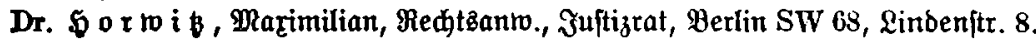

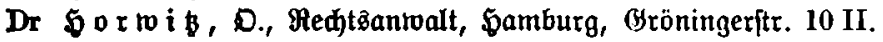

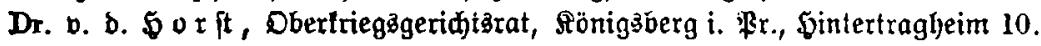




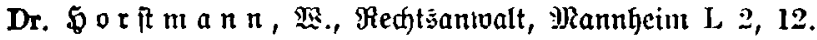

Dr. So e w i, 3 ., Redtsanwalt, Baben=Baben.

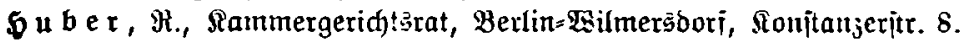

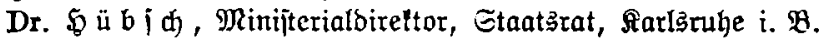

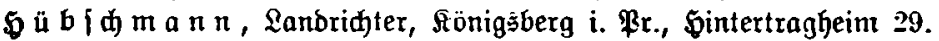

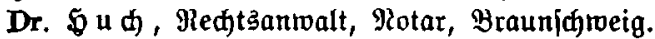

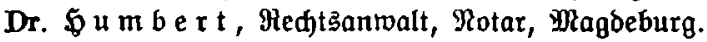

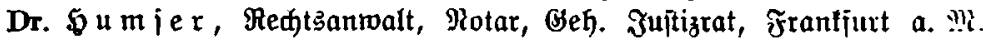

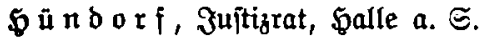

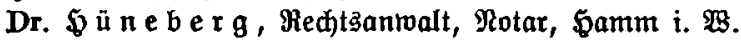

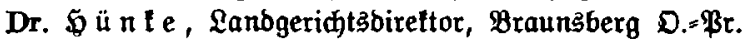

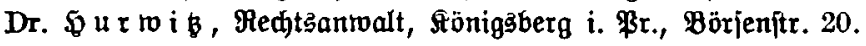

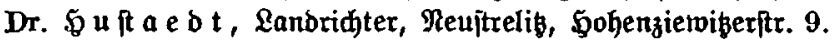

Dr. $\mathfrak{J} \mathfrak{c} \circ \mathfrak{b} i$, Ernit, Frofeffor, Müniter i. $\mathfrak{B}$.

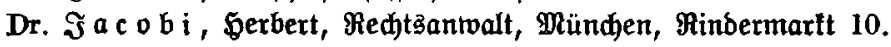

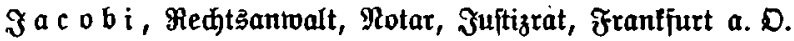

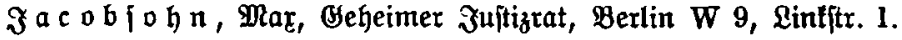

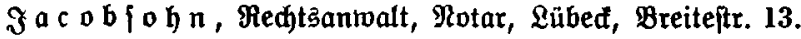

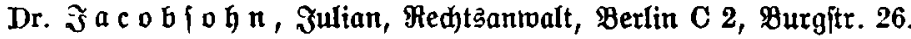

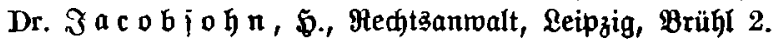

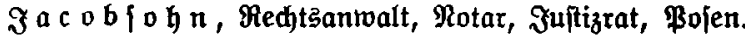

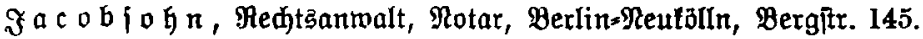

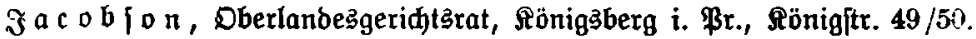

Dr. $\mathfrak{a} \subset \mathfrak{c} \mathfrak{b} \mathfrak{y}$, Sugo, Rechtsantwalt, Münden, Reuhaujerftr. 14.

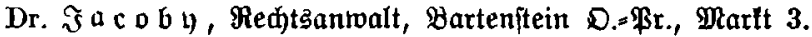

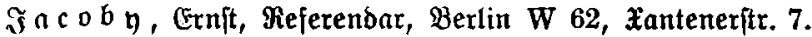

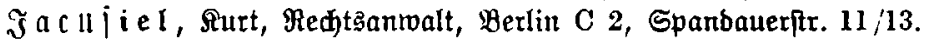

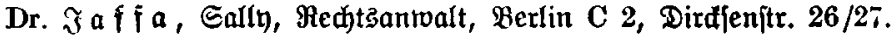

Dr. J a e ger, Otto, Notar, Godjelben.

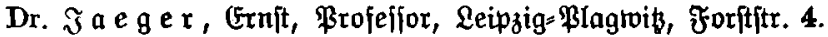

Dr. I a $\mathfrak{h} n$, Rechtsanwalt, Jultizrat, Brenzlau.

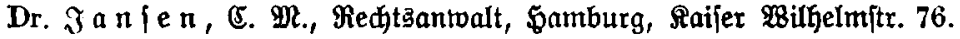

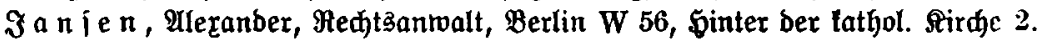

Dr. I a n в e n, Redtıantualt, Erefelb.

Jare $i$, Redtâantoalt, Pojen, Reue Straßje 1.

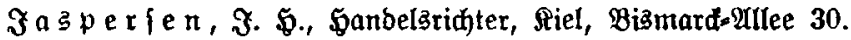

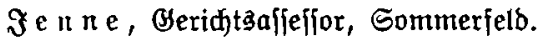

Эenzid, (E., Beh. Juftizrat, Sannwer, Böbeferftr. 21.

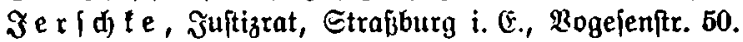

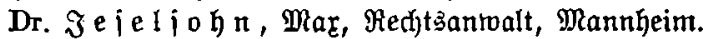

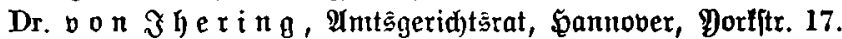

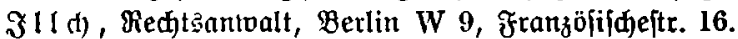

Imberg I, Franz, Ređtsantwalt, Berlin W 35, Botşameritr. 113, Billa 3.

Dr. $\mathfrak{J} \mathfrak{m}$ e $\mathfrak{r} \mathfrak{b} \mathfrak{a} \mathfrak{h}, \mathfrak{R} .$, Bantbireltur, Berlin W 15, Joadjimäthalerftr. 13.

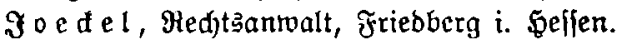

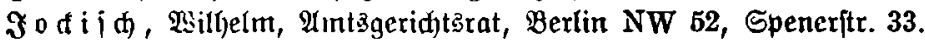

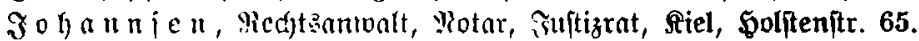




\section{XXXYI}

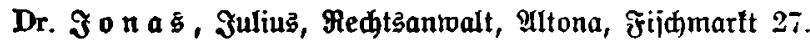

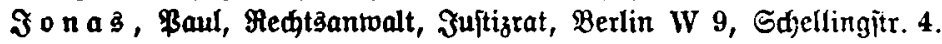

§oergens, Medtsanmalt, (Ejien a. Muhr.

Dr. Joerge 3 , Dr. jur. et philos., \alle a. E., Geebeneritr. 61.

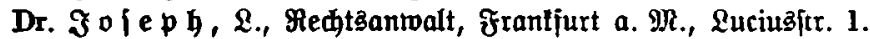

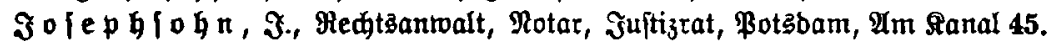

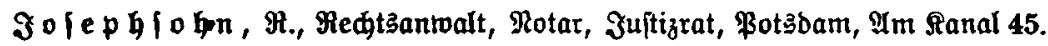

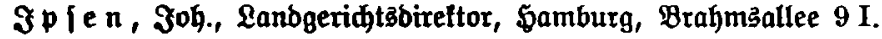

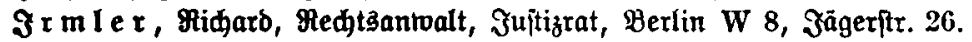

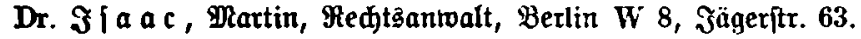

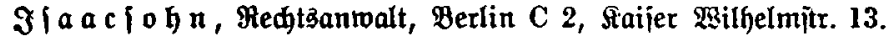

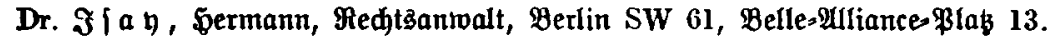

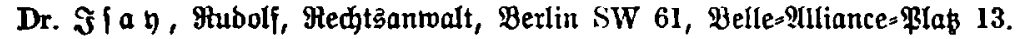

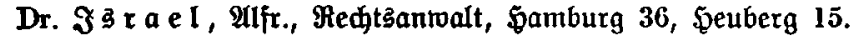

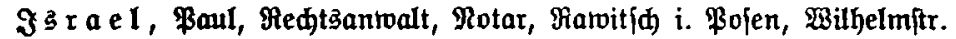

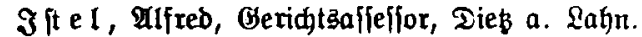

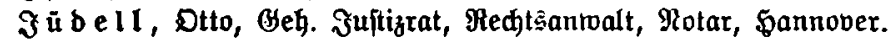

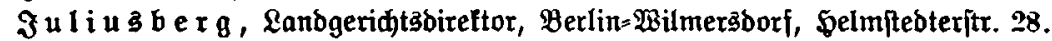

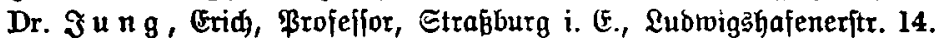

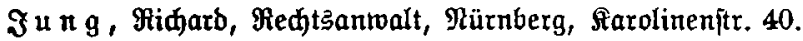

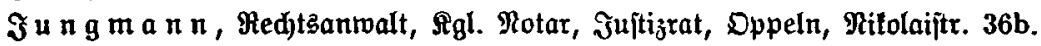

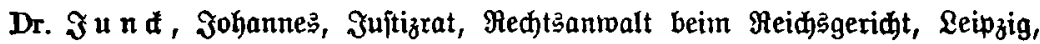
Ferbinand Ryodejtr. 38.

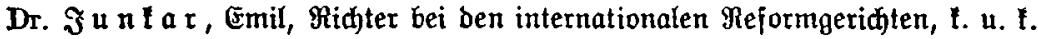

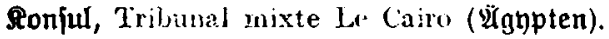

Suriftifdes Seminar ber Rgl. Wttbg. Eberharb Sarls=unniverjität,

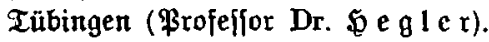

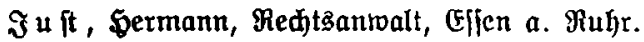

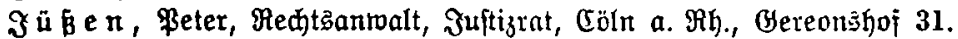

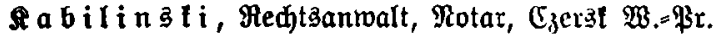

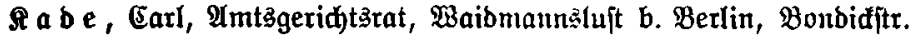

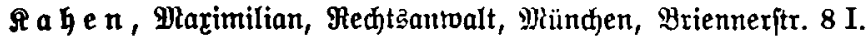

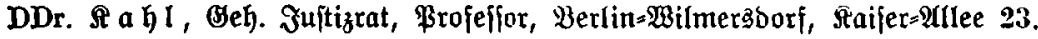

Dr. $\Re \mathfrak{a} \mathfrak{h} \mathfrak{n}$, Redtsantoalt, Mainz, Orr. Mleide 28.

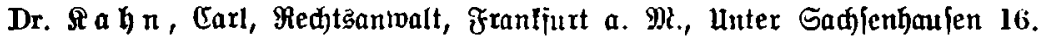

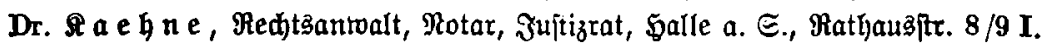

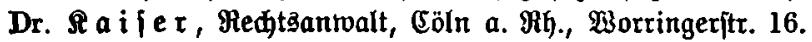

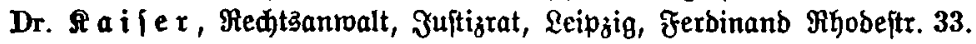

Dr. Ra ifer, Redtanantwalt, Mes, Briefteritr. 25.

M a 1 i $\mathbf{z}$ i , Max, Med̆tsanwalt, Breslau, Sd)weionitzerftr. 43.

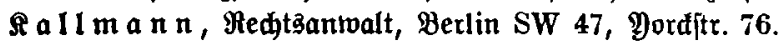

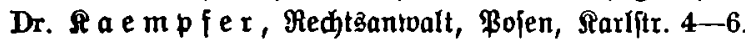

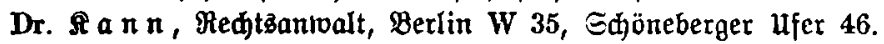

Dr. $\mathfrak{A} \mathfrak{n}$ torowiz, ferm. U., Pribatbozent, Freiburg i. B., Dreifönig. frape 30.

eapferer, Redtåninalt, (röln a. Mill. 


\section{XXXVII}

Is appe, Friebriđ, Redttäanvalt, Enndifuz der Groḡen Berliner Straßenbahn, Eharlottenburg 4, Santitr. 41.

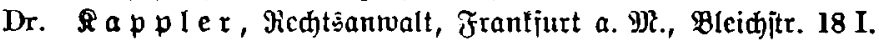

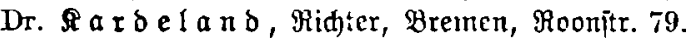

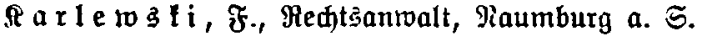

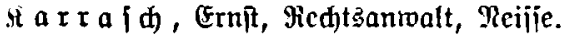

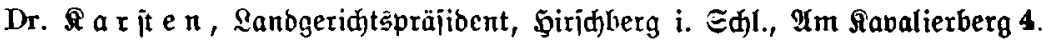

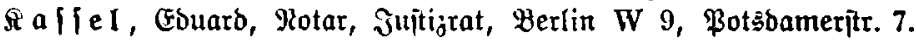

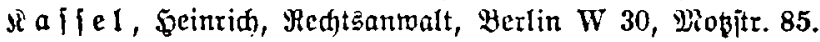

si a $\{$ fel, Dtto, Redtsanivalt, Ejien, a. Muhr.

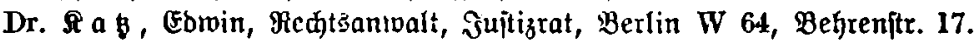

Dr. R a

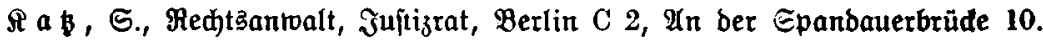

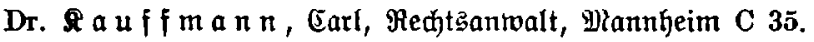

$\Re \mathfrak{a}$ if $\mathfrak{m a \mathfrak { n }}, \mathfrak{A r t h u r , ~ R e j e r e n d a r , ~ F r a n f i u r t ~ a . ~ M i l . , ~ B a ̆ r t n e r w e g ~} 20$.

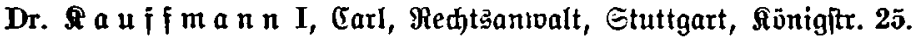

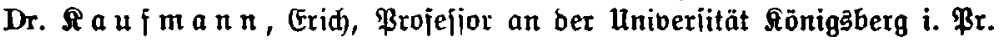

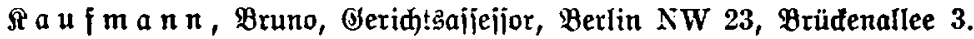

Dr. $\mathfrak{R} \mathfrak{a} \mathfrak{u} \mathfrak{f} \mathfrak{a} \mathfrak{n} \mathfrak{n}, \mathfrak{T h}$., Redtsanivalt, Seibelkerg, Bienenftr. 1.

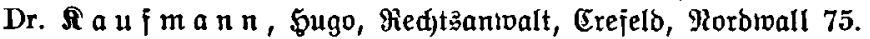

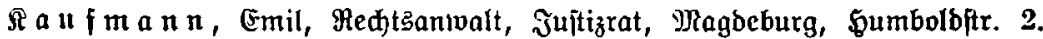

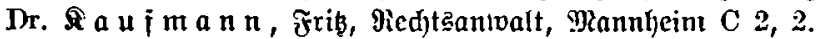

Dr. 尺a $a \mathfrak{f} \mathfrak{m} \mathfrak{n} n$, Gujtav, Red)t

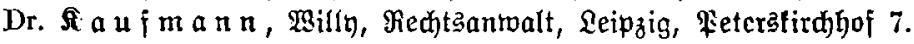

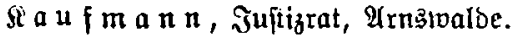

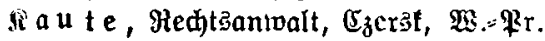

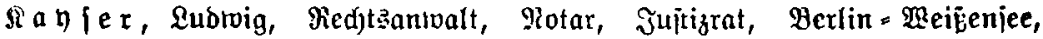
Berliner Alllee 17.

Re đ,

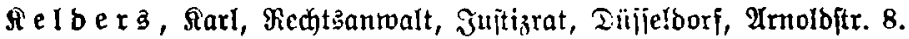

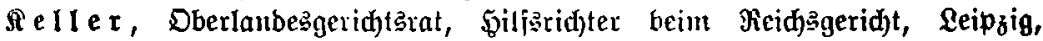
Ferbittand Rohbeitr. 14.

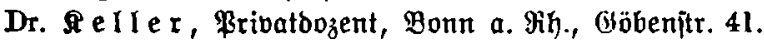

ReIlex, Lanbgeridgt3birettor, Mie 3baben, Merotal 55.

Dr. Re m me ter, B., Redtsanivalt, Juftizrat, Stempten i. $\mathfrak{B}$.

$\mathfrak{S e m p}\{$, Redtsanwalt, Juftizrat, Berlin W 64 , Unter ben Rinben 30 .

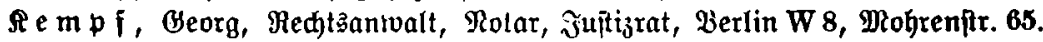

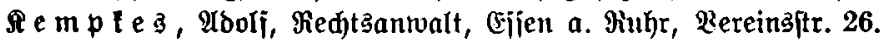

Rempner, Baul, Mejerendar, Berlin W, Ranograjenftr. 18 p.

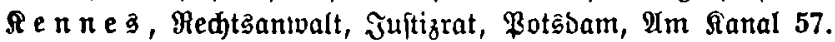

Ient, Baul, Juftizrat, Franfurt a. Mi., Taumaftr. 1 .

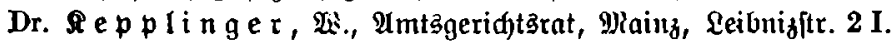

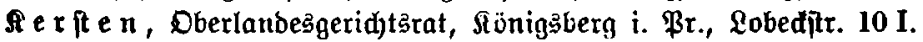

Serfting, Lanbgeridhtsbircttor a. D., Beh. Jujtizrat, Berlin= Behlenbori, Annenitr. 7.

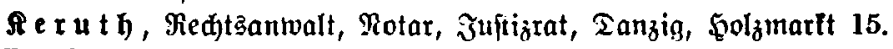

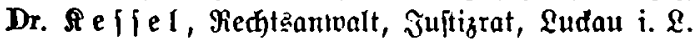




\section{XXXVIII}

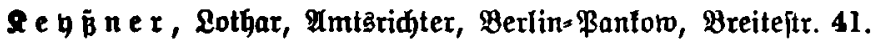

Dr. $R$ i e f e I,

A i f f er, Bedtzantoalt, Colmar i. E.

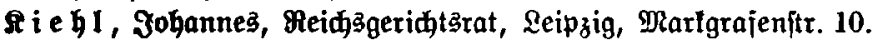

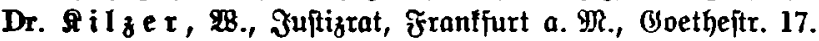

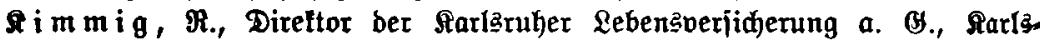
rube i. 8 ., Raifer-2lllee 4.

Dr. $\mathfrak{i}$ in $b, \Re .$, Düffelborf, Sdumannitr. 4.

R inbel, Willh., Senatzprâfibent, Geheimer Obersłuftizrat, Berlin W 62, Bayreutberftr. 35.

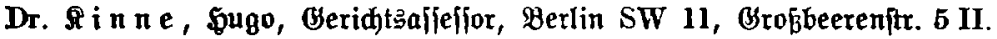

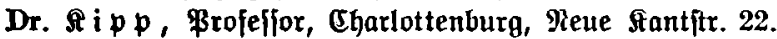

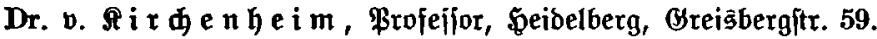

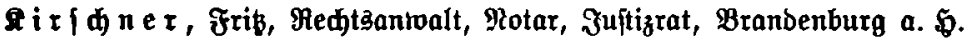

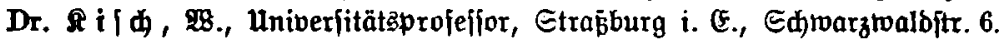

$\Re$ i ß , Sanogeriḑtäpräjibent, Grejelb.

Dr. $\Re$ i в i n ger, Friebr., av. Univerfitätäprojejior, Mündhen, Tengftr. $13 / 0$.

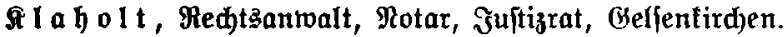

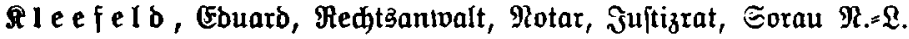

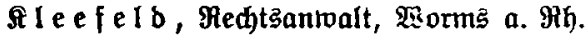

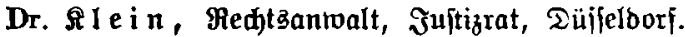

Dr. RI e in f ell ex, Bs., Brofefior, Siel, Riemannsıeg 101.

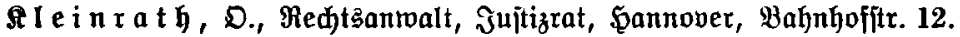

Dr. N I e n t e r, Mbolf, Međtsanwalt, Elberfelb, Wortmannftr. 43.

Rloeppel, Friebrid, Rechtantwalt, Dresben=Blaferwiţ.

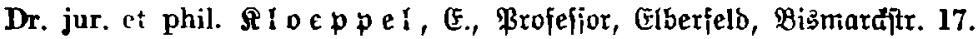

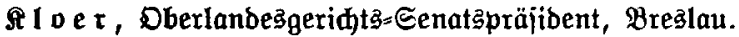

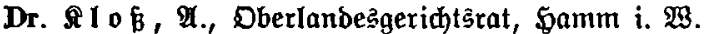

$\mathfrak{A} \mathfrak{\mathfrak { u } g}$, Beorg, Beridtzaffelfor, Danzig, Langenmartt 41 II.

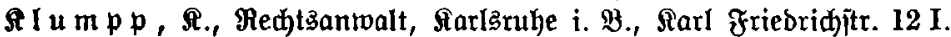

$\Re \mathfrak{I}$ ü $\mathfrak{f} \in \mathbb{I}, \mathfrak{Q} .$, Finanzrat $\mathfrak{a}$. D., Stuttgart, Dannecteritr. 21.

Dr. $\mathfrak{\Re} \mathfrak{a} u$ er, Lanbgeridytsbireftor, Samburg 20, Eppendorfer Ranbjtr. 70.

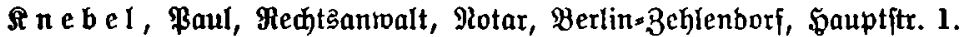

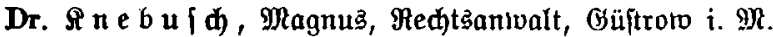

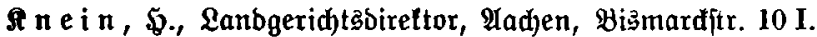

Dr. Iniep, (3eh. Jujtizrat, Brofeffor, Jena.

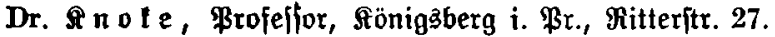

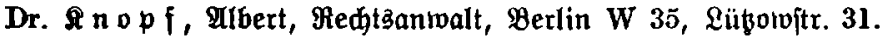

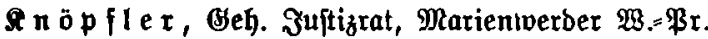

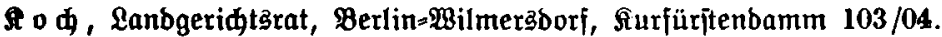

$\Re \circ$ 中, Max, Redtanantwalt, Berlin W 9, Botżamerttr. 13.

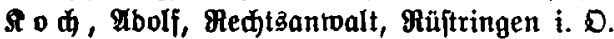

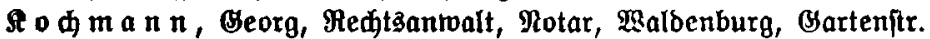

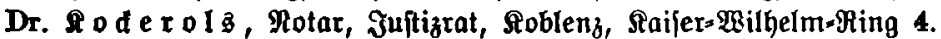

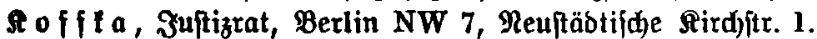

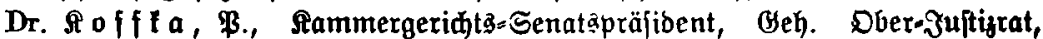
Berlin NW 23, Siegmunbahof 16. 


\section{XXXIX}

Dr. $\mathfrak{A}$ o e $\mathfrak{h}$ I e $\mathfrak{r}, \mathfrak{B}$., Redtsanwalt, Dannheim.

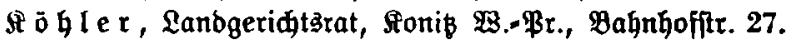

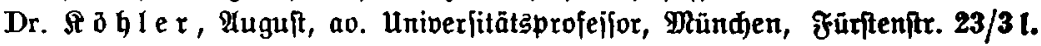

Dr. $\mathbb{A} \circ \mathfrak{h} \mathfrak{i} \mathfrak{a} \mathfrak{u}$ i d, , F., Brofefior, Gtrāiburg i. E., An ber $\mathfrak{A} a r 14$.

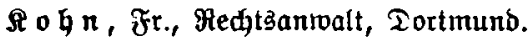

Dr. jur. et phil. $\mathfrak{A} \mathfrak{o} \mathfrak{h} n e$, Carl, Brofeffor, Berlin W 62, Reithftr. 12.

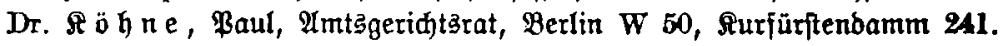

Roelle, Mmtsgeridgtszat, Böttingen.

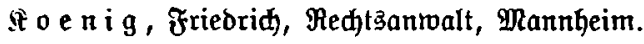

Dr. R o n i g b e $\mathfrak{g}$ er, Paul, Lanbridter, Berlin W 30, Speneterftr. 18.

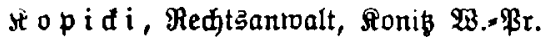

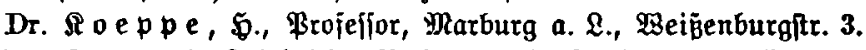

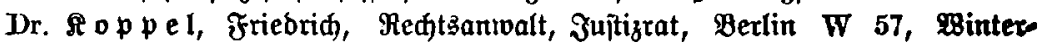
feld traß̄e 4.

$\mathfrak{F} \ddot{p} \mathfrak{p} \in \mathbb{I}$, Redtsanwalt, Jultizrat, Belgaro (Pommern).

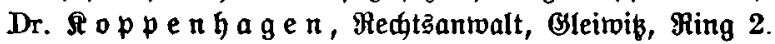

A o e r b i b, Mmts̄ridter, Berlin W 50, Mantejtr. 21.

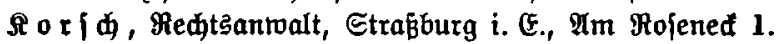

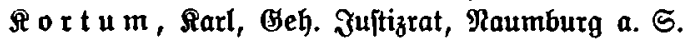

$\mathfrak{A} \ddot{o} \mathfrak{f}$ e r, Lanogeridtżpräfibent, Memel, \&ibauerftr. 18.

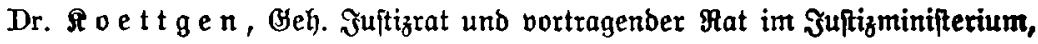

Ber in W 15, fturfürftendamm 197/98.

$\mathfrak{S} x \mathfrak{a} \mathfrak{h}$, Dberlanbes̉geridtşrat, Celle.

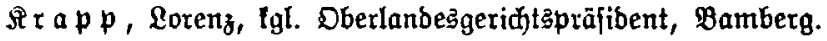

Dr. $\Re$ r a $t \mathfrak{t}, \mathfrak{F}_{r}$, Redtsantralt, Bforzheim.

Dr. Araeme $r$, Wilfelm, Medhtananwalt, Berlin W 35, Botsbamerftr. 00 .

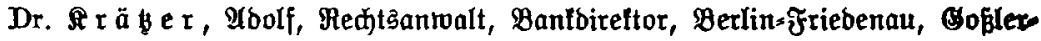
prape 9.

Dr. A $\mathfrak{x} \mathfrak{a}\{e$, Bseh. Juftizrat, Berlin W 64, Behrenftr. 24.

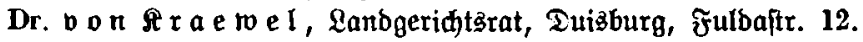

Arei (d), Seo, Medftanmalt, Bonn, Medenheimerftr. 44.

Sienzer, Theodor, Dberantsrifter, Subrigsitadt (Bayern).

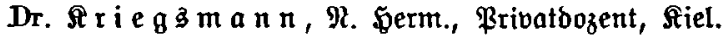

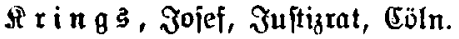

Dr. $\mathbb{R}$ 吅 $\mathfrak{n} e, \mathfrak{B e r l i n} W 50$, Rürnbergerjtr. 25/26.

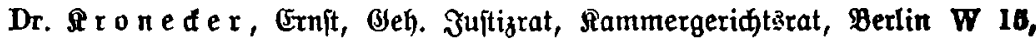
Gdlüterftr. 40.

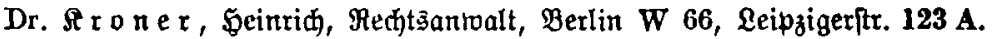

Dr. A r onfel b, Redjtantwalt, Juftizrat, Berlin W 8, Taubenitr. 14.

Dr. Aronfel b, Juftizrat, Weimar.

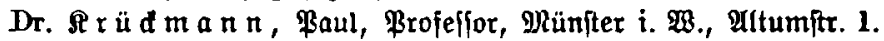

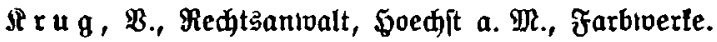

Dr. $\mathfrak{A} \mathfrak{r} \mathfrak{u} \mathfrak{g} \in \mathfrak{r}$, E., Dozent, Ealtw.

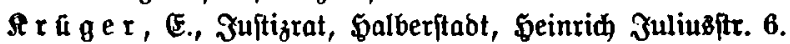

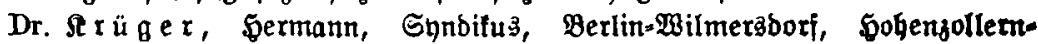
bainm 190.

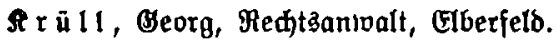




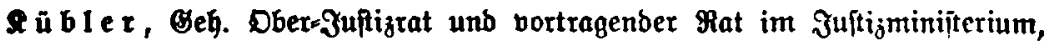

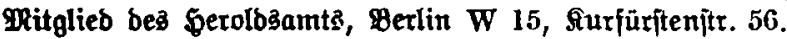

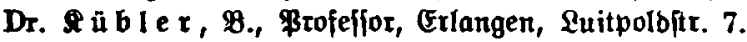

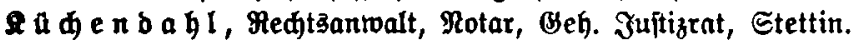

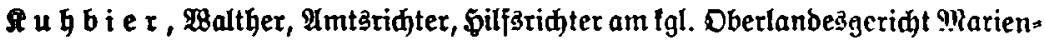

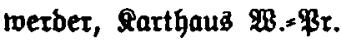

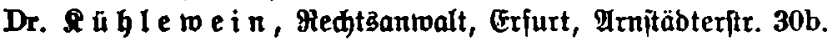

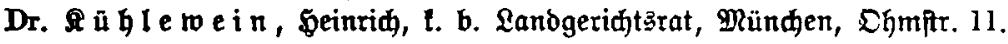

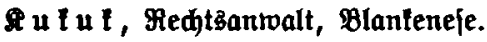

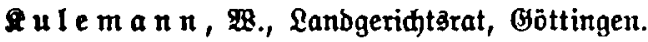

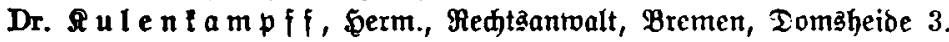

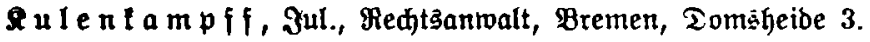

Dr. $\mathbb{Q} \mathfrak{a} \mathfrak{l}$, Medtşanroalt, Marburg a. $\mathfrak{Q}$.

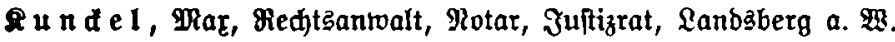

Dr. b. $\Re \tilde{u} \mathfrak{n} \mathfrak{\beta} \mathfrak{b} \in \mathfrak{r} g$, Eberhard Freiferr, Eeibelberg, Bergitr. 53.

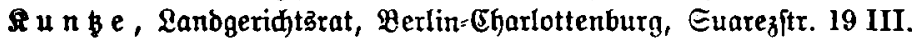

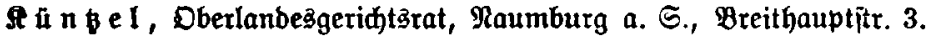

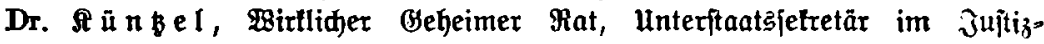
minifterium, Erzellenz, Berlin W 35, Benthineritr. 39.

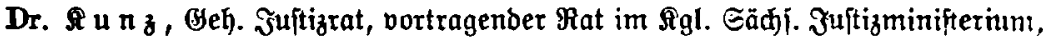
Dreşben=凡., Düppelitr. 1.

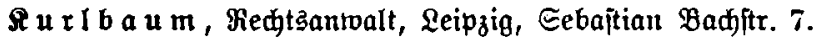

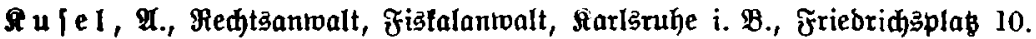

Dr. A ü $t \mathfrak{t}$ er, Lanbridjter a. D., Pribatbozent, Berlin=Grunervalo, \$Bjgmannftr. 6.

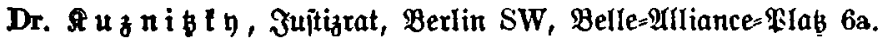

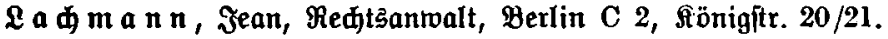

Dr. I a d e $r$, צlfonä, Meferenbar, Breălau, Bartenitr. 109.

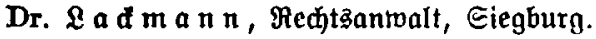

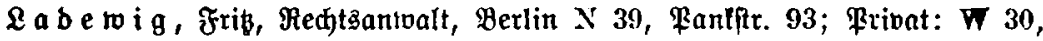
\$ohenftaufenftr. 52.

$\mathfrak{E} a \mathfrak{g} \mathbf{0}$, Fechtsantwalt, Bexlin W 66, Maucrftr. $78 / 79$.

$\mathfrak{L}$ ä $\mathfrak{h}, \mathfrak{A m t z g e r i d j t z ̇ a t ~ a . ~ D . , ~ S e i b e l b e r g , ~ \Re o ̈ m e r i t r . ~} 58$.

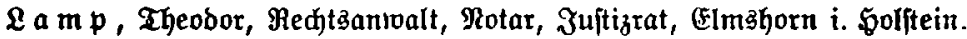

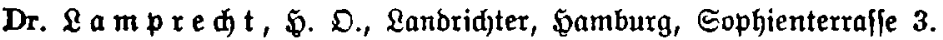

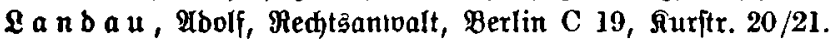

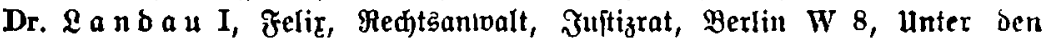
Linben 39.

Ia n bé, Imtäridter, Berlin W 15, Dlinaerplaß̧ 9.

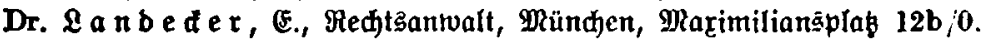

I a n b e $r$, Ferbinanb, Lanogeriat şbirettor, Bofen.

$\mathfrak{L} a \mathfrak{n} b \mathbf{m} a \mathfrak{n}$, Stabtjynbifus ber Stabt Mannheim.

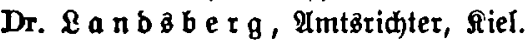

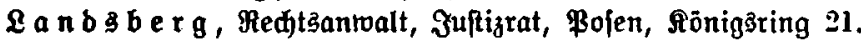

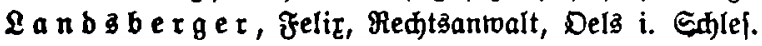

\& a $\mathfrak{n}$, $\mathscr{A m t 3 r i d t e r , ~ \Re o m b a d ~ i . ~ S o t h r . ~}$

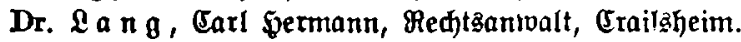


La nge, Ređtsanwalt, Jujtizrat, Straßßburg i. (5. Am Rojened 1.

Dr. La $\mathrm{g}$ e $\mathrm{n}$, Brofeffor, Breifşwalb.

Dr. \&ange $\mathfrak{n} \mathfrak{b} a$, Geridtsafieffor, Worms, Groetheftr. 15 .

I a n ger, Lanogeriđjtşbirettor, Ratibor, Wilkelmitr. 10.

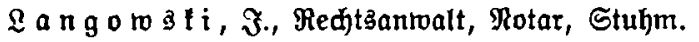

La $\mathbf{s}$ ter, Juptizrat, Lanbaberg a. 2 .

Dr. \&a 3 ter, Rejerenbar, Breălau, Bartenitr. 109.

$\mathfrak{a} \mathbf{u}, \mathfrak{J u f t i z r a t , ~ T a n z i g . ~}$

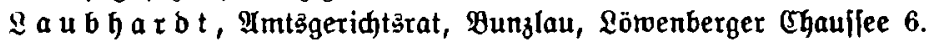

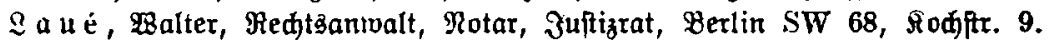

Dr. Le a n ber, Reøtzanwalt, Berlin SW 68, Zimmeritr. 19.

Dr. $\mathfrak{L}$ e $\mathfrak{b}$ i $n$, Geheimer Juftizrat, Berlin SW 68, Friedriditr. 44.

Dr. $\mathcal{L}$ e b i $n$, Ernit, Redtşanmalt, Berlin SW 68, Friebridftr. 44.

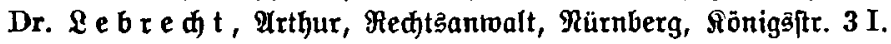

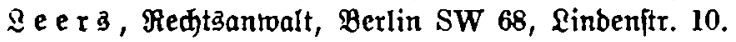

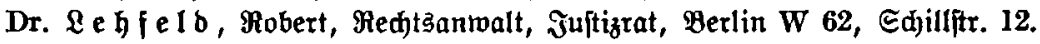

Dr. Leh m a n n, Staatsantoalt, Sharlottenburg, Ließ̨enburgeritr. 11.

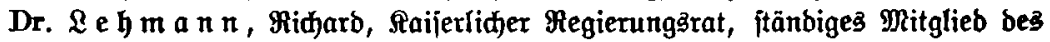

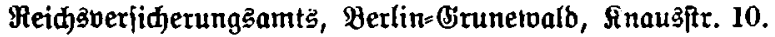

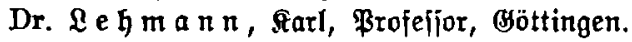

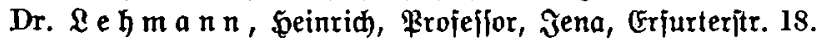

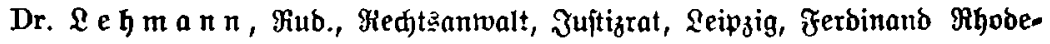
frape 30 .

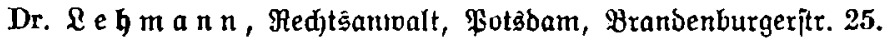

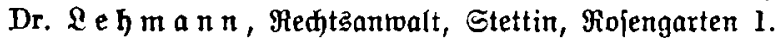

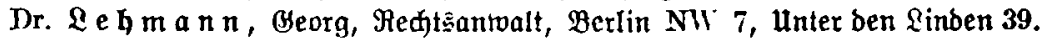

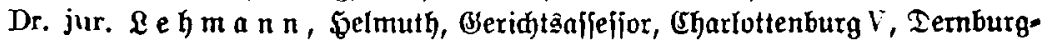
itrá̧e 28.

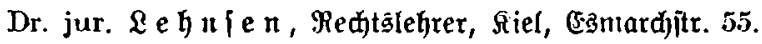

Dr. Le i b i g, Regierungşrat a. D., Rrofeffor, Berlin W 15, Bfalzburgerftr.72a.

Dr. L e ip z i ger I, Beorg, Medtsanvalt, Breslau I, Junlernjtr. 14.

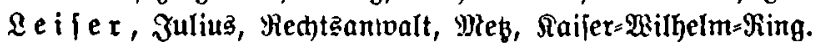

Dr. $\mathfrak{L}$ e mberg, $\mathfrak{A r t f}$ ur, Redt graben 10 .

Le m $\mathfrak{l}$ e, Georg, Umt3ridter, Magdeburg, Đohepfortejtr. 46.

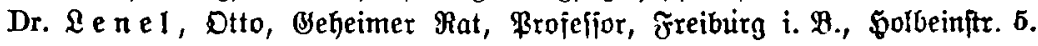

$\mathfrak{L}$ e $\mathfrak{h}$ a $\mathbf{b} \mathrm{t}$, MIfreb, Lanbridter, Miesbaden, Meubauerftr. 3.

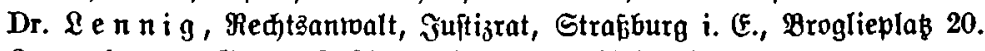

\&e nzberg, Bleorg, Jujtizrat, Đannover, Anbreäftr. 9.

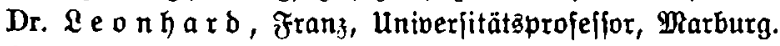

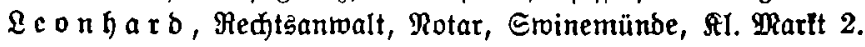

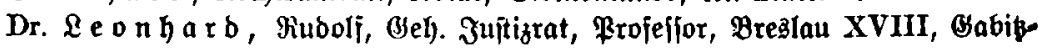
frá̧e 188.

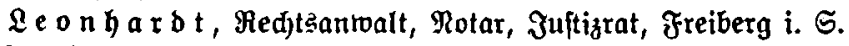

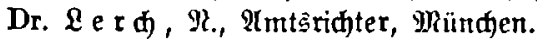

Dr. Le fer, Guibo, Beridteaffeifor, beibelberg, Bergftr. 32.

Dr. Le fer, walter, Oberamtäribter, Mannheim $\mathrm{L}, 4,1$. 


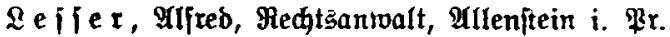

Dr. Le f f e $r$ Martin, Medtsantwalt, Bojen, Friebriditr. 3la.

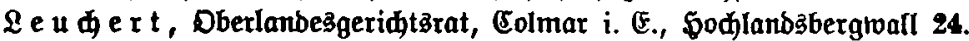

Dr. Le $\mathfrak{i}$, M., Medtbantwalt, \&ltona a. E.

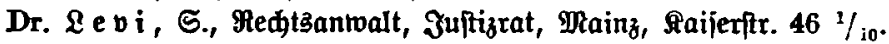

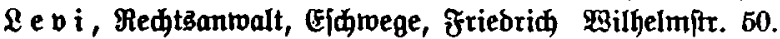

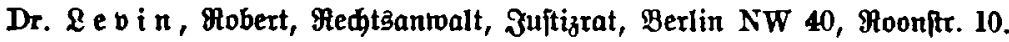

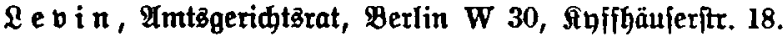

Dr. Le viz, Dtto, Oberamtëridter, Bforzheim, Linbenitr. 17.

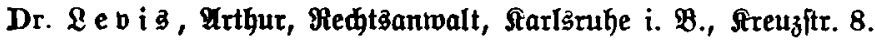

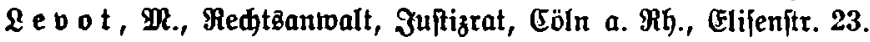

Dr. Lev $\mathfrak{y}, \mathfrak{3}$. A., Mboofat, Amfterbam, Meeresigradt 210.

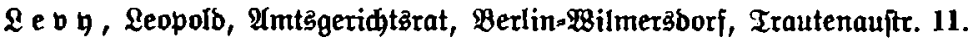

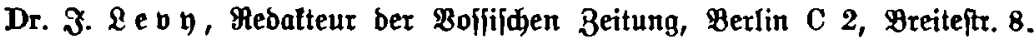

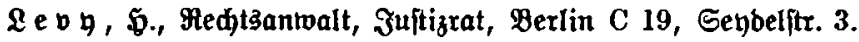

\& e v , Julius, Medtzantwalt, Berlin C 2, Stralmerftr. 13/14.

Dr. $\mathcal{L}$ e $\mathfrak{v} \mathfrak{y}$, Hedtananalt, cafiel.

Dr. $\mathfrak{L}$ e v $\mathfrak{y}, \mathfrak{A r t h u r , ~ \Re e c h t s a n t w a l t , ~ B e r l i n ~ S W ~ 6 8 , ~ F r i e b r i d i t r . ~} 208$.

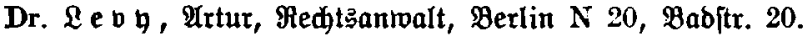

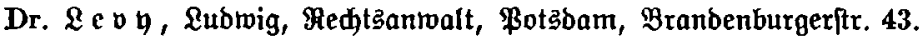

Dr. $\mathfrak{L}$ e $\mathfrak{y}$, Medtzanmalt, Eifer.

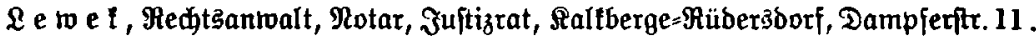

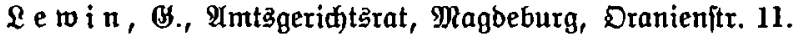

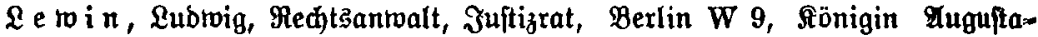
itraje 19.

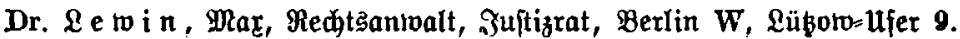

Dr. Le to i $n$, Redtitantwalt, §uftizrat, Stettin, Breiteitr. 33.

Dr. \&ewinsti, Mechtsantwalt, Rotax, Juftizrat, Gharlottenburg, Fafanenjtx. 83.

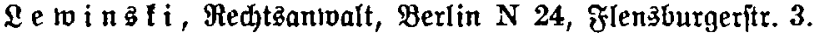

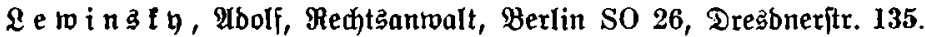

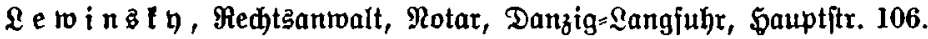

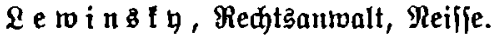

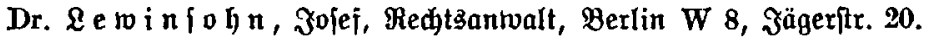

Dr. Le q $\{$ є

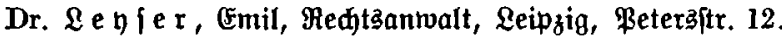

Ley $\{$ er $\{$ on, Redtzantwalt, Juftizrat, Berlin SW 68, 3immeritr. 5/6.

Dr. $\&$ i d $t$, Stabtrat, Berlin=Sdjöneberg, Meranerftr. 3.

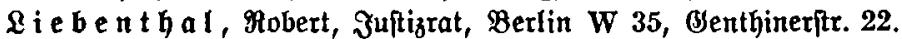

Dr. $\mathcal{L}$ i e $b$ f ne d) $t$, Rarl, Redtsantwalt, Berlin $N$ 4, Ehauffeeftr. 121.

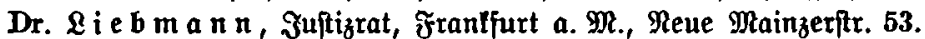

Dr. \& i e b m a $n \mathfrak{n}$, Dtto, Gerauşgeber ber Deut|đen Suriftenzeitung, Berlim

W 67, Botabamerftr. 96.

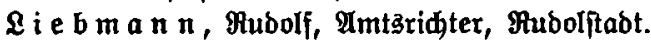

Dr. L i e o $f$ e, Medtantwalt, Rotar, Flensburg.

\&ie b t le, Medtazantwalt, Berlin W 35, Blumeshgof 12.

Dr. Li e f $m$ a $\mathfrak{n} n$, Robert, Brofeffor, Freiburg i. $\mathbb{B}$., Boetheftr. 33.

Liepmann, Profeffor, Riel, Moltfeftr. 42. 


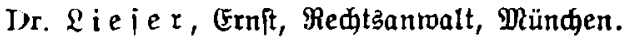

Q i e c m a n $\pi$, Redtsantwalt, Friblar.

Dr. v. Lil i e $\mathfrak{n}$ th al, Marl, Brofeljor, Beheimer \$ofrat, \$eibelberg, Biegel haujer Lanbitr. 30.

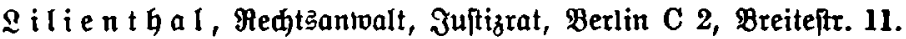

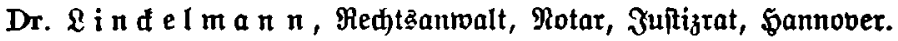

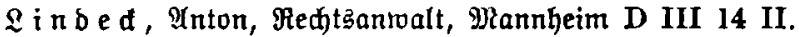

I i n Qnzbaderitr. 15.

Dr. $\mathfrak{R}$ i n $t$, Rat am Stabts und Ranbamt Rübed, Barabe 1 , Sdgló Manbau.

Dr. $\mathfrak{L}$ i o $\mathrm{n}$, Ređtżantwalt, Berlin W 8, Reipzigerftr. 98.

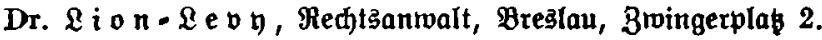

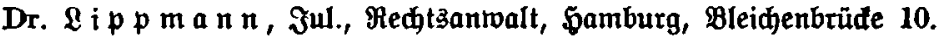

L i s c o, Juftizrat, Berlin W 8, Mrauerftr. 37.

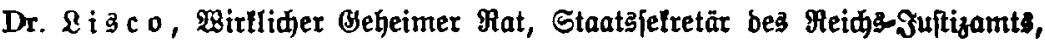
Exzellenz, Berlin W 9, Bopitr. 5.

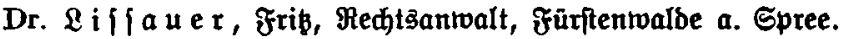

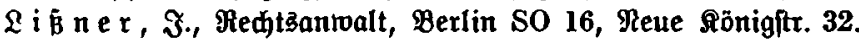

Dr. v. $\mathcal{L}$ i $z_{z} t$, franz, Beh. Suftizrat, ßrofeffor, Eharlottenburg, \$arbenbergftrape 19.

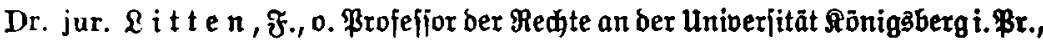
SaitaniensAllee 38.

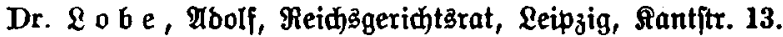

Lo e b e I I, Redtianmalt, Salberitabt.

Dr. $\mathbb{L}^{\circ}$ ff $\mathrm{e} r, \mathfrak{E} .$, Redtsanmalt, Colmar i. E., Sienmannftr. 2.

Lo h in a n $\pi$, Lanbgeridtsbireltor, Blanteneje-Dodenhuben, Boetheftr. 10.

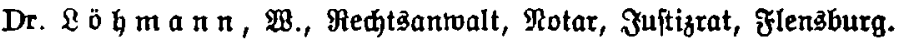

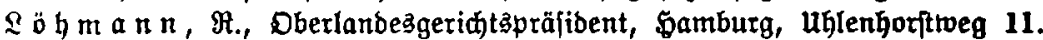

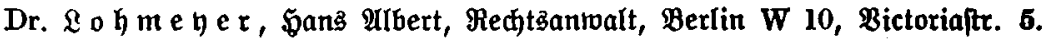

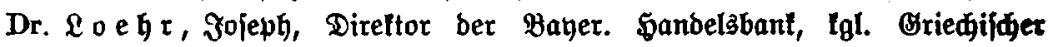
Bלneralfonjul, Münđen, Mfabemieftr. 11.

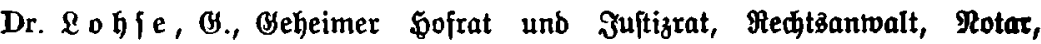
Leipzig, Matharinenftr. 20.

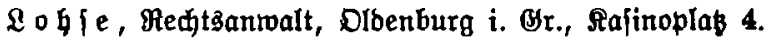

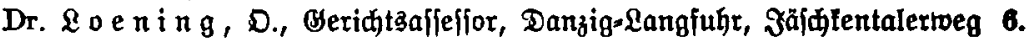

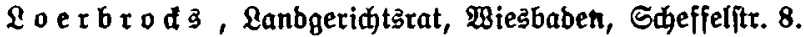

Dr. $200 \mathbb{f}$, Lanbridter, (5harlottenburg, Sdillerftr. $127 \mathrm{III}$.

\&orenz, Rammergeridtgrat, Berlin W 30, \&uitpolbftr. 13.

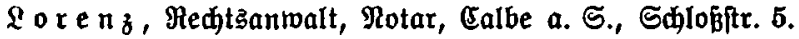

Dr. jur. \&orenz, Emil, Redtsantwalt, Berlin C 2, Epanbauerftr. 48.

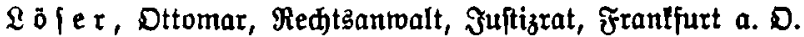

Dr. Ro e infon, Martin, Redtsantwalt, Juftizrat, Berlin W 35, Mragbeo burgeritr. 22.

Dr. Lo e we , Jofef, Medţantwalt, Berlin W 8, Taubenftr. 43.

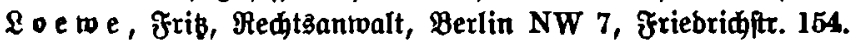

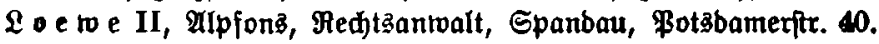




\section{XLIV}

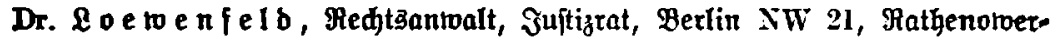
fraje 78.

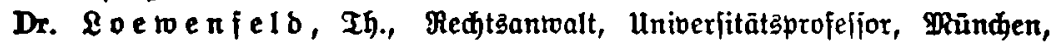
Pfanbhausftr. 31.

Io e we nfte in, Mectsanmalt, Raberborn i. 2 .

Lo ewe nftei $n$, Medtsanwalt, Notax, Dberhaufen (Mgib.), Marttitr. 77.

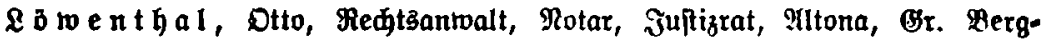
frape 266 I.

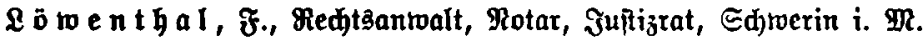

Soe twe nthal, Mar, Redtantwalt, Berlin W 8, Sanonier|tr. 8.

L o e to i $\{$ o $\mathfrak{n}, \mathfrak{F}$ tanz, Medtsantralt, Brešlau, Gutenbergitr. 47.

Dr. \& o e wy , Emil, Mechtsanmalt, Juftizrat, Berlin W 8, Leipzigerftr. 19 II.

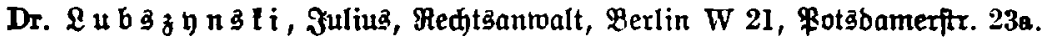

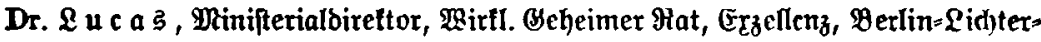
felbe, Ihellaftr. 11.

\& ủ \& e rath, (א., Medtsantralt, Rotar, Miettmann.

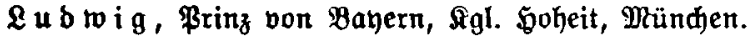

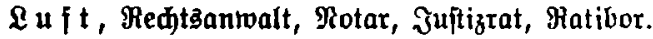

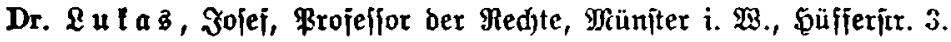

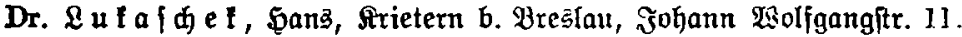

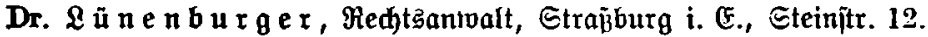

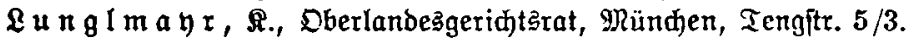

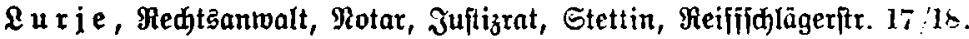

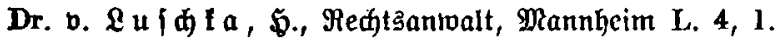

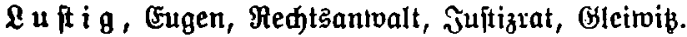

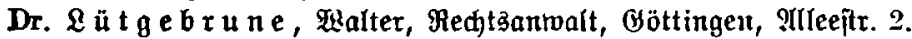

$\mathfrak{L}$ ü $\mathbf{t}$ e $\mathbf{m} \mathfrak{a} \mathfrak{n}, \mathfrak{B}$., Rotar, Sannover, Beorgąplaß 10.

Q ü в e I e r, Mug., Raijerl. Rotar, Maašntüniter.

Dr. Ma a $\mathfrak{s}$, Beorg, Brofeffor, Shifolasfec b. Berlin, Potzonmer Shaujice.

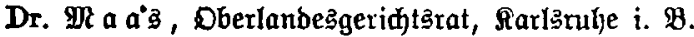

Dt a $\mathfrak{g} \mathfrak{n}$ 3, Jultus, Redtsెantwalt, Berlin NW 7, Dorotheenitr. 41.

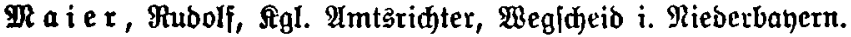

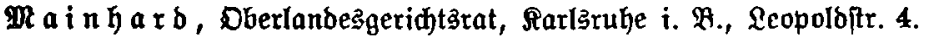

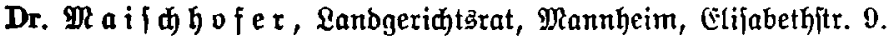

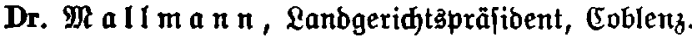

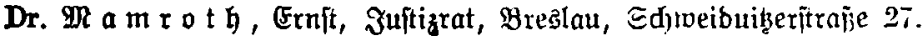

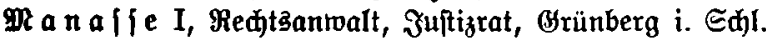

Dr. phil. et jur. $\mathfrak{M}$ a $\mathfrak{n}$ e 3 , Brofeffor und Generaljelretär bes beutidhen Ber.

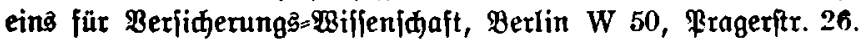

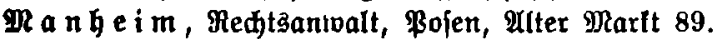

פR a nhe i mes, Juftizrat, ßojen 0 , Friebridfitr. 5.

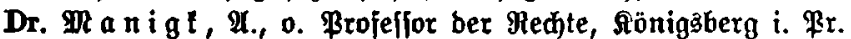

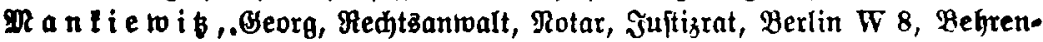
frape 14-16.

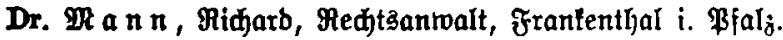

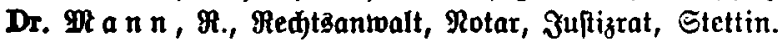

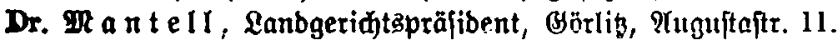




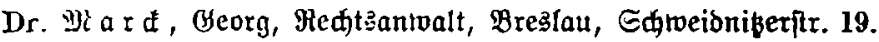

Ir a $\mathfrak{b} a$, Redtżanwalt, Berlin W 64, Behrenftr. 68/69.

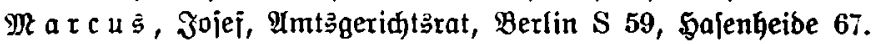

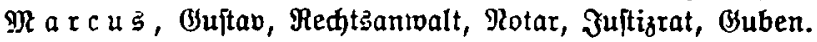

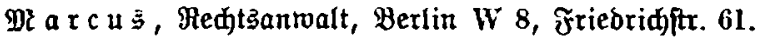

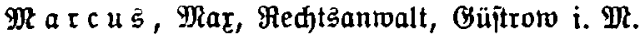

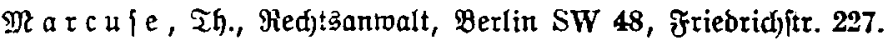

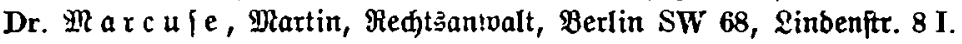

$\mathfrak{R}$ a $\mathbf{c}$ u $\{e$, Dšmalb, Jujtizrat, Breälau.

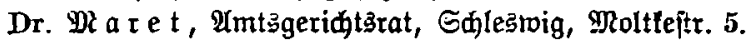

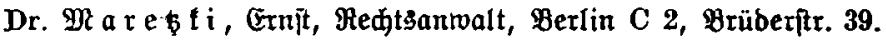

Ma a i or bing , Fuitizrat, Paberborn.

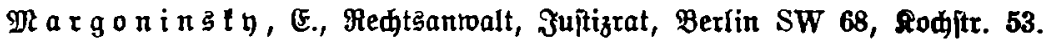

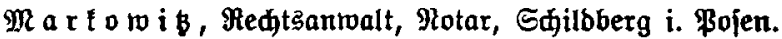

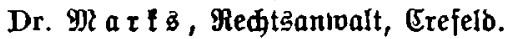

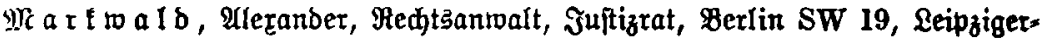
iträ̉e 83.

Dr. Martin, M., Eenatşprājioent, ફamburg 37, Berberftr. 42.

o e $\mathbb{M} a r t$ inc o u $t$, Geridtalaffeffor, Soldin, Mememannitr. 23.

Dr. MR a rtini, Surt, Reøtzantwalt, Berlin W 9, Rintftr. 18.

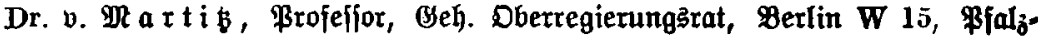
burgerftr. $85 / 86$.

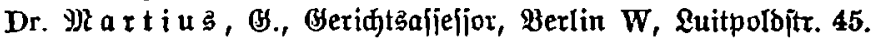

$\mathfrak{M}$ a $\mathbf{x}$ u $\mathbf{m}$, Lubrig, Redtsantwalt, Sarlsmbe i. $\mathfrak{B}$.

Dr. Ml a $r$ tw i $b$, Oberlanbesgeridtstat, Riel.

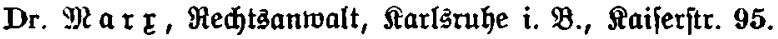

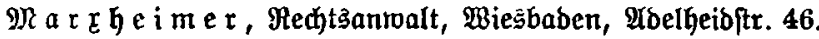

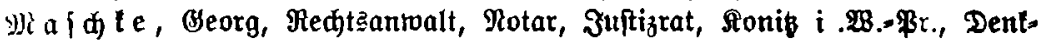
malsplaz 7 .

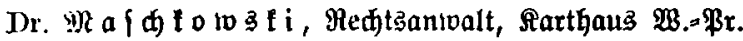

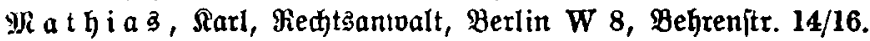

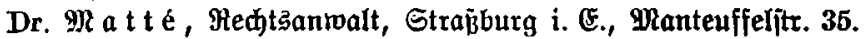

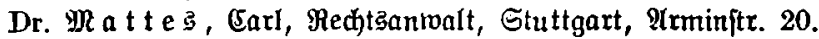

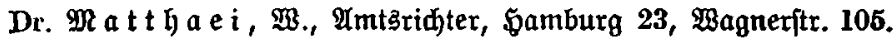

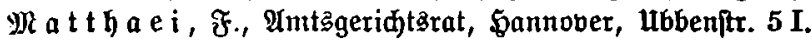

Dr. Mat thi a 3 , Brofeffor, Moftod i. MR., Stefanitr. 13a.

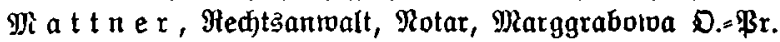

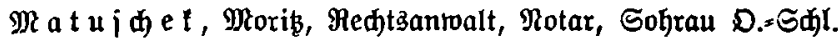

$\mathfrak{M} \mathfrak{a} \mathfrak{u} \mathfrak{b} \mathfrak{a} \mathfrak{d}$, Ghutab, Motar, Jultizrat, Roblenz.

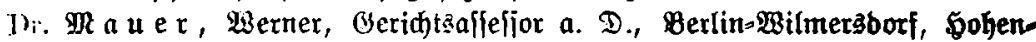
zollernbamm 201.

Yi a u r, Ređtsantwalt, Juftizrat, Coblenz a. Rh.

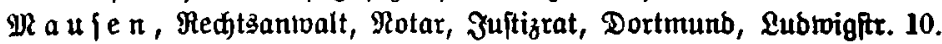

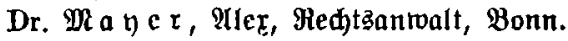

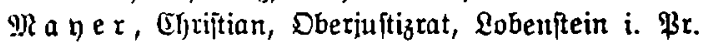

Dr. $\mathfrak{M}$ a y e r , (Earl, Med)t

Dr. Ma yer, Moribs, Merbtiantwalt, Frantentyal i. Mfalz. 


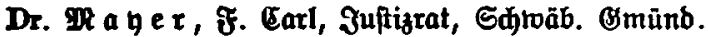

Dr. PR a yer, Maz Emft, Frofeffor, Etrapburg i. E., Drangeriering 19.

M a yer, Bembarb, Redtsantwalt, Strabburg i. E., Blautoollengaffe :.

Dr. IR a yer, Guftav, Redtzantwalt, MRannheim.

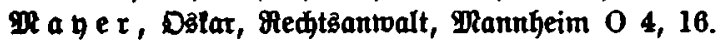

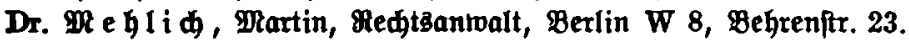

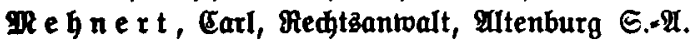

IR e i b a u e, , Medtsantwalt, Motar, Juftizrat, Flatow $23 .=$ \$r.

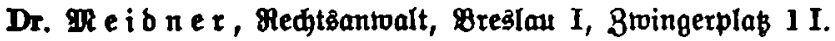

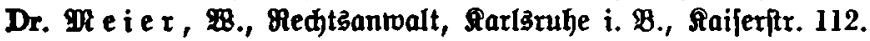

Dr. Di e i e r, S., Medtzantwalt, Altona a. E.

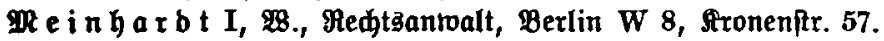

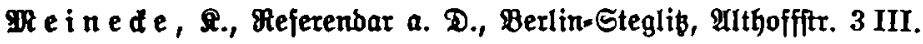

Me in in ger, Bhilipp, Medt 3 anwalt, Mainz, Bahnhofplab 2.

IR e in z inger, Redtzantwalt, Lanbau.

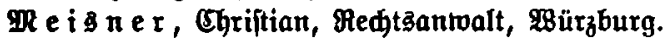

Di e i ́ n ex, Siegfrieb, stub. jur., Berlin=Steglik, Bimmermannftr. 21.

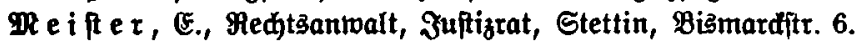

Dr. Mt e I đi or, Beorge, Medtsantoalt, bamburg, Br. Bleiden 23.

Dr. Mt e n $\delta$ e 1 , Redhtsantwalt, Dreaben, Maridallftr. 27 I.

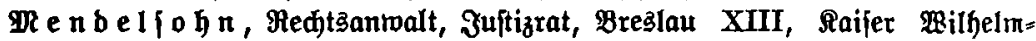
Prape 106.

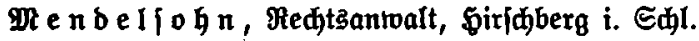

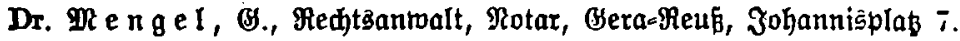

Re n ge 1 berg, Redtęanivalt, Suftizrat, Crefalb.

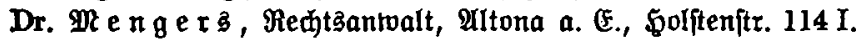

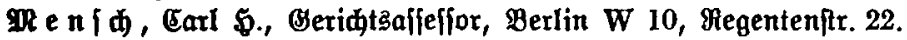

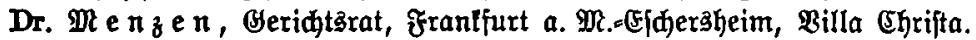

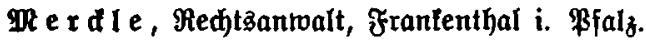

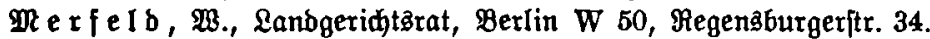

Dr. MR e r e I, Paul, Brofelfor, Greifzrwalb, Steinftr. 11.

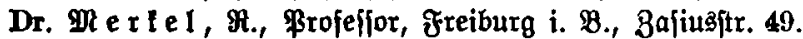

Me $x$ t e $\mathfrak{n}$, Mectitzantwalt, Bromberg.

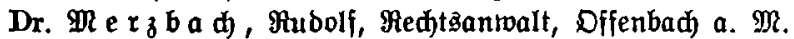

$\mathfrak{M}$ e $\mathbf{z} \mathfrak{b} \mathfrak{a}$ d $\in \mathbf{r}$, Rectsantwalt, Juftizrat, Rürnberg.

Dr. Me 低elfohn, Juftizrat, Berlin W 10, Stülerftr. 6.

Me $\{\{$ o wo, Mrfreb, Mechtzantwalt, Juftizrat, Berlin C 2, Burgftr. 26.

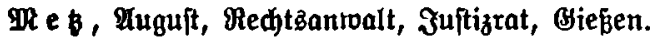

Dr. If e b e I, Bribatgelehrter, Stettin, Linbenftr. 21, p. I.

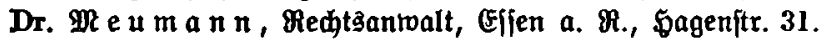

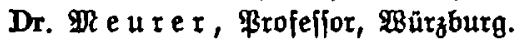

פe u fe 1, Beorg, Mmtżridter, Blauen i. B., Saiferftr. 18 I.

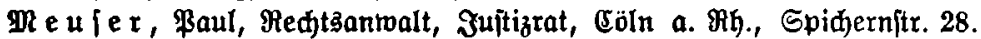

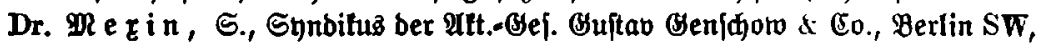

(Sharlottenftr. 6.

Dr. med. et jur. Me yer, $\mathfrak{I h}$., \$ribatbozent, Jena, Gerberftr. 3.

Dr. $\mathfrak{P}$ e yer, $\mathfrak{Y}$ riß̧, Jultizrat, Franffurt a. $\mathfrak{M}$., Zeil 63 . 
Me yex, Dtto, Dberlandeâgeriđtärat, Gelle, Berggartenitr. 21.

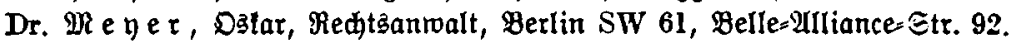

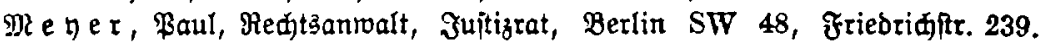

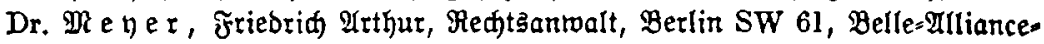

Plaks 3.

MR e ver, Bernfaro, Redtsantwalt, Rotar, Branbenburg a. $\$$.

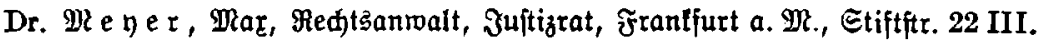
Me yer, Eugen, Medtsantralt, Freiburg i. $\mathfrak{B}$.

Dr. De y e r III, \&ubwig, Rectşantwalt, Đamnover, Edilleritr. 32.

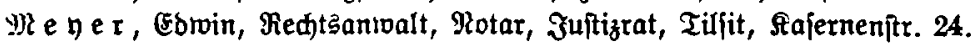

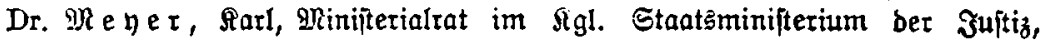

Pründen, Serdenjelbitr. 7.

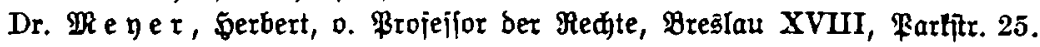

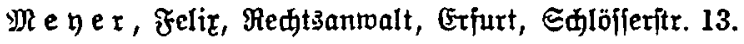

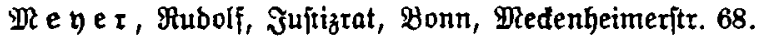

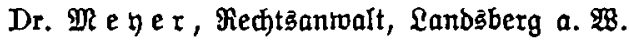

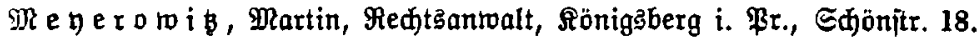

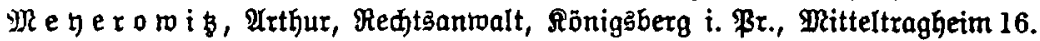

Megertitein, Ebuarb, Rechtsantwalt, Syndifus ber pandelstammer, Berlin NW 7, Dorotheenitr. $7 / 8$.

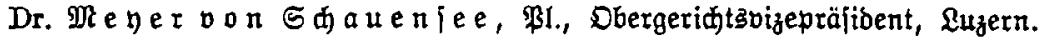

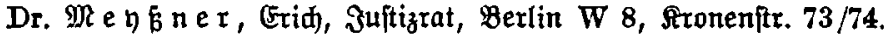

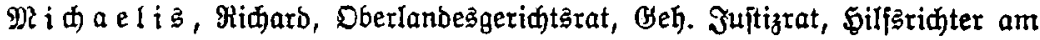

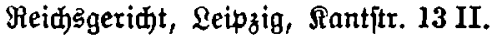

Dr. Mi đa eli 3 , Mar, Redtzantwalt, Berlin W 64, Eharlottenitr. 59.

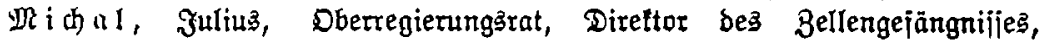
গürnberg, Fürtheritr. 114.

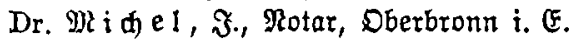

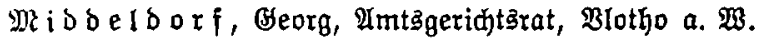

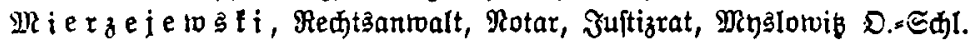

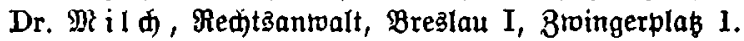

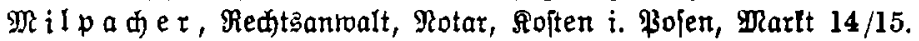

Dr. Mi i n b e $n$, Beorg, Direttor bes Piandbriefamtes, Berlin W 62, STeiftitr. 1.

Dr. $\mathfrak{R}$ i $\{$ क , Emil, Ređtsantwalt, Juftizrat, Berlin C 2, Reue Friebridftr. 71.

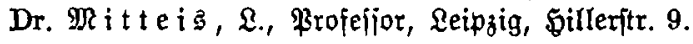

Dr. Mit te lit a e $b t$, Meditsaninalt, Leipzig, Sd)entenborfitr. 4.

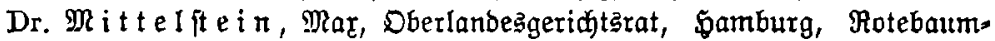
d)aufiee 34 II.

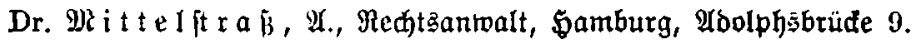

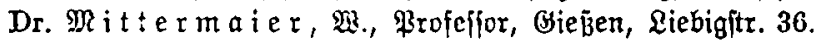

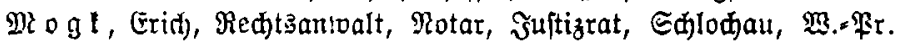

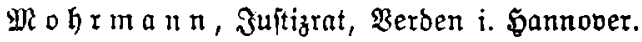

Dr. MRoll, 5., Juftizrat, Perlin W 35, Jar\}ăab 26.

$\mathfrak{M}$ on inger, Earl, Brantereibireftor unb Ganbelłridter, Farlärube i. B., Friebenitr. 1.

Dr. $\Re$ O o

von mor 


\section{XLVIII}

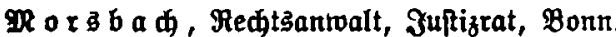

M o je 3, Beorg, Medtszantwalt, Leipzig, Burgitr. 1-5.

M o le s, Midarb, Redtsanwalt, Berlin W 66, Maueritr. 4.

Dr. $\mathfrak{R}_{\mathrm{R}}$ \{ 3 , Siegfrieb, Bexlin NW 23, solfteiner ufer 12.

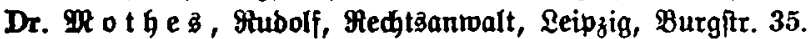

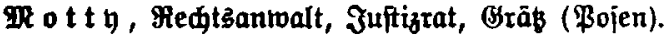

PR of $\mathfrak{t} a$, Fedtsanwalt, Rotar, Loeben.

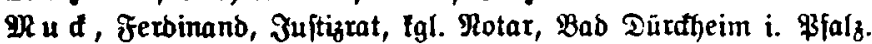

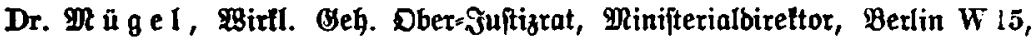

Turfürftenbamm 32; i. Sommer: Nicolaßjee, Cimbernftr. 12.

Dr. $\mathfrak{R}$ й

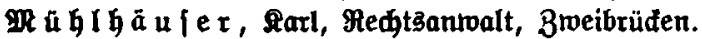

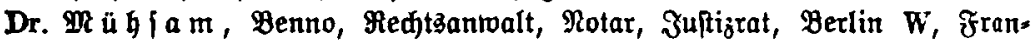
zölifieltraje 16.

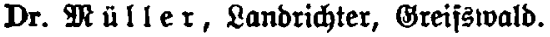

Mï lle r, MeҺtzantwalt, Notar, Juftizrat, Demmin.

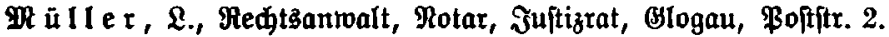

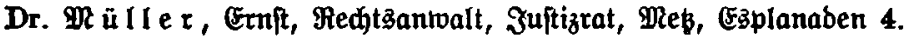

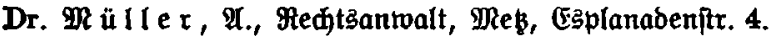

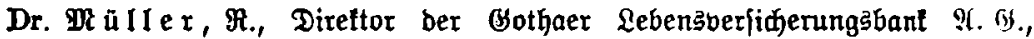
Bsotha.

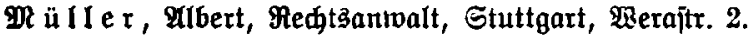

Dĩ Il e r, Eberharb, Medtłantwalt, Berlin W 66, Maueritr. 94.

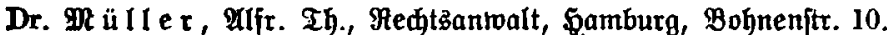

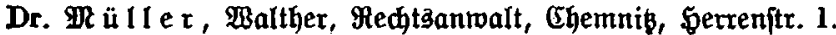

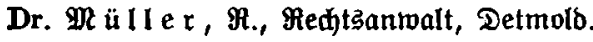

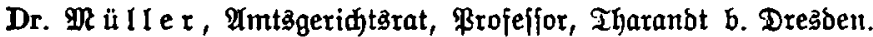

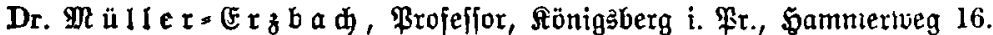

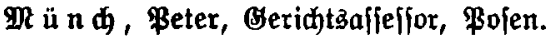

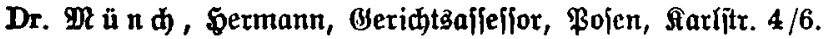

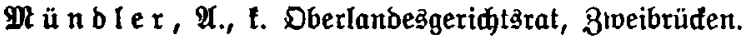

IR $u$ n b $t$, Mmtšridter, Danzig, Sandgrube 28 .

Mu nl, Ranbgeridhtsoireftor, Geh. Suftizrat, Berlin×Brunewalb, Cronberger. itrape 24.

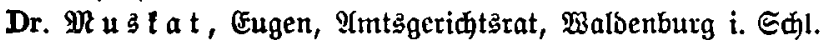

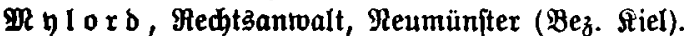

Dr. $\mathfrak{R}$ a gle $r$, Johann, \$rofelfor, Bafel i. Sabweiz, Sonnentweg 21.

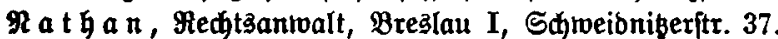

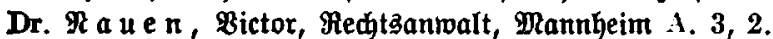

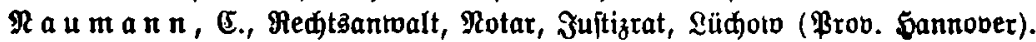

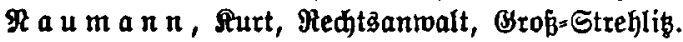

$\Re$ e a e l, Leopolb, Dberlanbez̉geridt

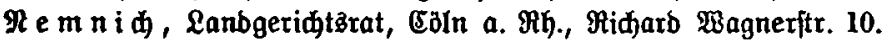

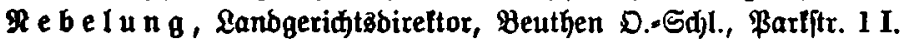

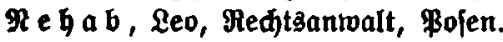

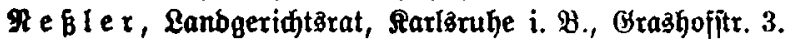




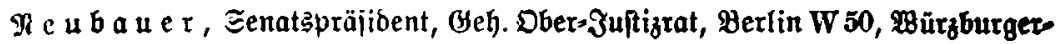
ftrāe 3.

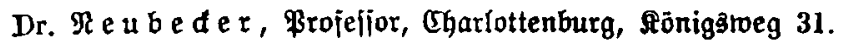

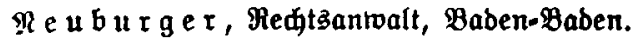

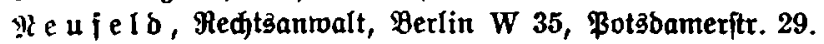

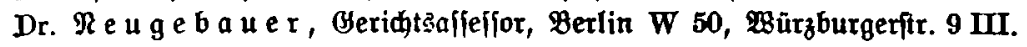

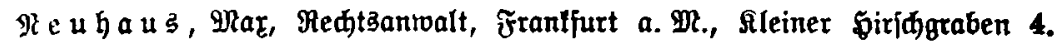

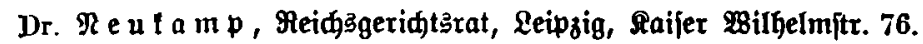

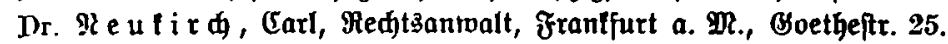

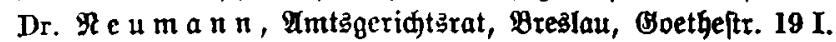

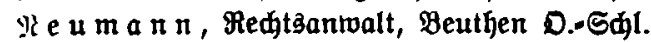

Yi e u m a n $n$, Bictor, Jultizrat, Notar, Breșau II, Gartenitr. 58.

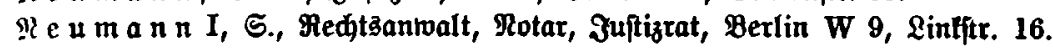

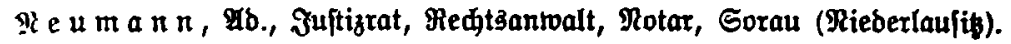

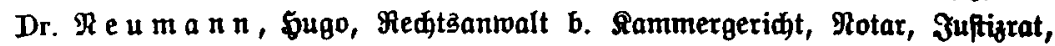
Berlin W 35, Bot3bamerftr. 118.

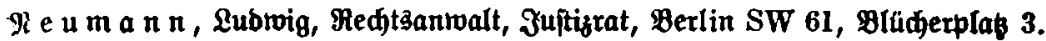

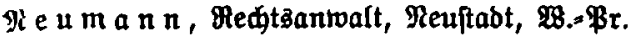

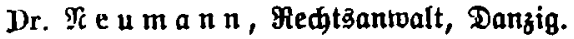

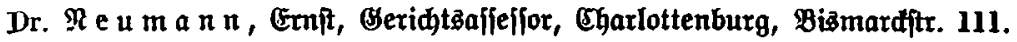

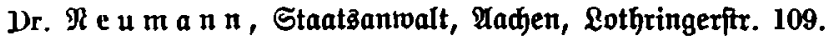

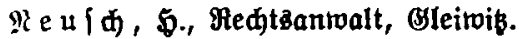

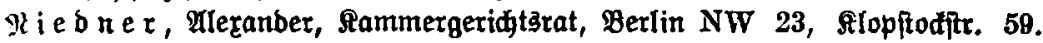
])r. Mi emeyer, Brofelfor, Riel.

Dr. Ri e nu e yer, Bictor, Juftizrat, Gffen.

])r. Ri i i c, Otto, befolbeter Sdjöffe, Berlin-Treptow, Im Treptotver \$art 21. yt ic je, Mted)tsantoalt, Rotar, Juftizrat, Miel, Solftenitr. 104.

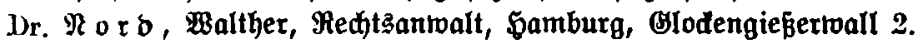

Dr. Mor b heimer, Max, Amts̈ridter, Uerbingen b. Exefelb.

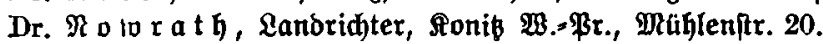

Dr. $\mathfrak{R}$ u $\mathfrak{3}$, Bilhelm, Redtsanmalt, Dortmunb, Ditenhellweg 11.

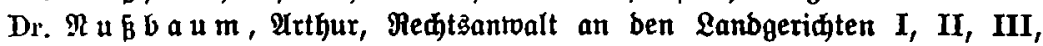
Berlin W 62, હđilliptr. 2.

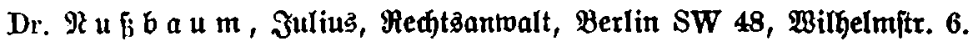

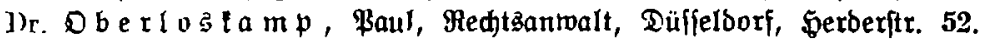

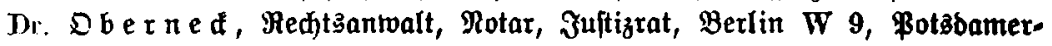
ftrapie 134b.

Obre d) $t$, G., Suftizrat, Deb, Bantitraßse.

Ober $\mathbf{z} \mathfrak{t} \mathfrak{y}$, Juftizrat, Breslau, Ming 17.

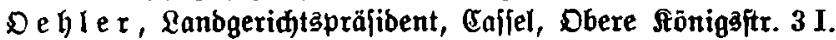

Oh $n$ fit e in, Mechtsantwalt, Berlin W 60, Tauenbienitt. $7 b$.

$D \mathfrak{b} r i$ d $t$, Exfter Etaatsantwalt, Qüneburg.

Dr. Dele nhe inz, Rehtşantwalt, Rarlşruhe i. B., Rmalienjtr. 24.

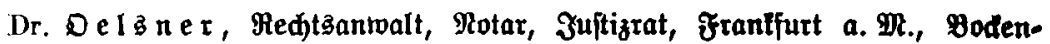
Geimer Sanbitrabe 2.

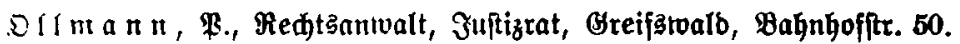




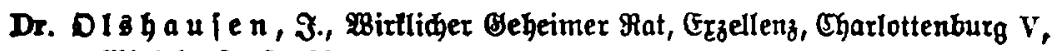
Biblebenftrake 31.

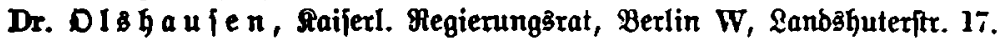

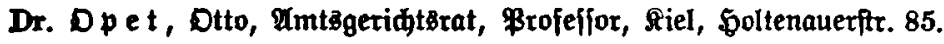

Dp pen he im, Bubolf, affeffor, Berlin W 10, Friebrid Milfelmftr. 8 .

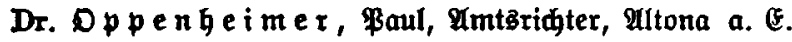

Dppenheimer, Mentsantoalt, Darmftadt.

Dr. Dp pe ń с i mer,

Dr. Dppenhe imer, E., Redtzantwalt, Juftizrat, Berlin W 8, Regenten. frape 2.

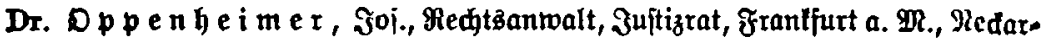
Prafe 10.

Dr. Dp pen

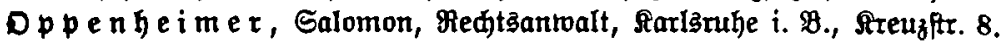

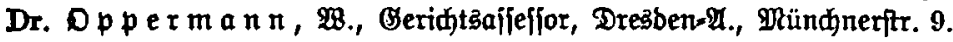

Dr. Oppermann, Reidjżgeriđtšrat, Leipzig, Egelitr. 5.

Dppler, Leo, Dberlandes̈geridtsెrat, Colmar i. E., Bartholdiftr. 5 a.

D g g 1 r, Furt, Redtsantwalt, Barmen.

Dr. Orgle $r$, Rehtsantwalt, ßojen.

Dr. Drtli eb, Geridtåajeffor, Berlin NW 52, গirdfitr. 11 bei Sdamiot.

Dr. jur. et phil. Dert ma $\mathfrak{n} n$, Baul, Brofeffor, Erlangen.

Dr. Drt we il e $x$, Redtsantwalt, Meiningen S.SM.

Dr. jur. Ofter ri eth, \$rofeffor, Sduriftiteller, Berlin W66, 23ilhelmftr. $57 / 58$.

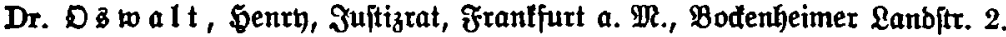

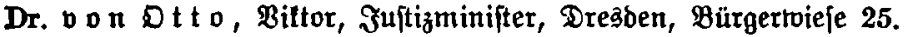

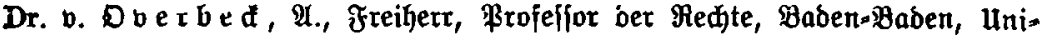
verjitätä̧traß̧e 6 .

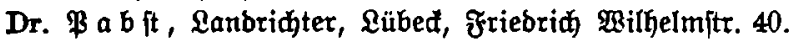

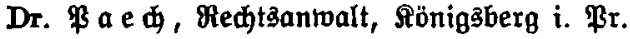

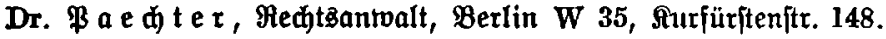

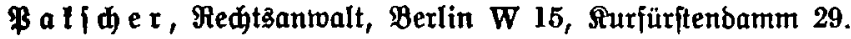

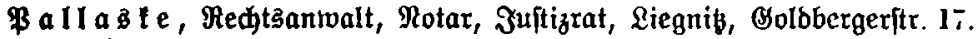

Dr. Ia pe, (5rnft, Rammergeridjtzrat, Berlin W 15, Fonftanzerftr. 52.

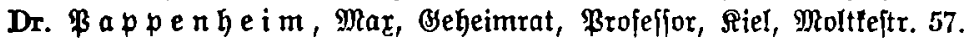

\$a rtifel, Medtäantwalt, Berent, $\mathfrak{W} .=$ Br.

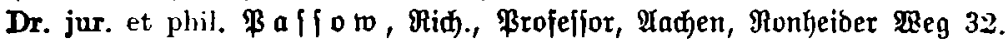

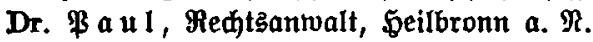

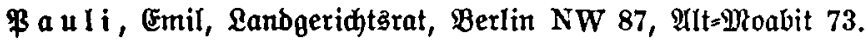

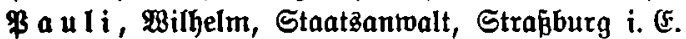

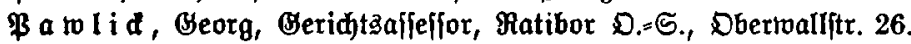

ь о MRünđen, Б̧eridjelftr. 16.

Be i ne, ., Regierungsat, Baberborn.

Dr. ße ipel mann, B., Reditzantwalt, Mitenburg S.st.

Be if e r, Georg, Feđtşantwalt, Breslau I, Junfernftr. 11 II.

Belt a $10 \mathfrak{h} \pi$, Raul, Beridtsaffeffor, Berlin W 15, Sonjtanzerftr. 68.

B e me $\mathfrak{r l}$, Friebrich, tgl. banr. Oberamtätichter, Mündjen, Sicbigitr. 7 . 
Dr. \$e r e I3, Leopolb, Brofeffor, beibelberg, Brüdenftr. 39.

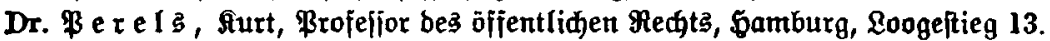

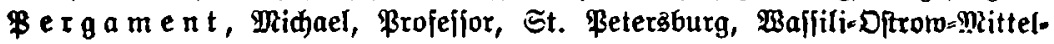
Bropett 56.

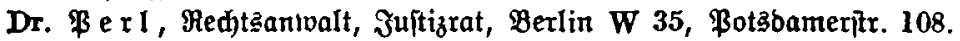

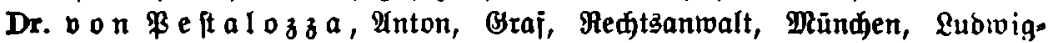
ftrabe 26.

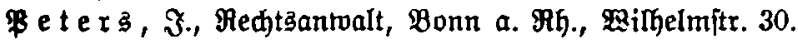

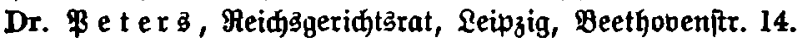

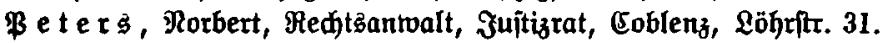

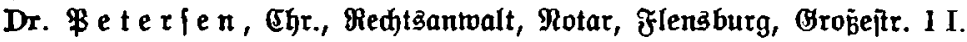

Be trid), Medtâanwalt, Rotar, Jultizrat, Samter.

B e a l I, Betholb, Medtzantwalt, Berlin C 2, Dirdienftr. 26/27.

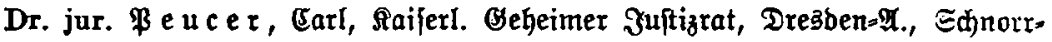
frake 72.

B faffrath, Eenatsprālibent, Colmar i. (E.

Dr. Bfefferle, Bilhelm, Beridtsaffefior Ftarlaruhe i. B., Bunjenitr. 3.

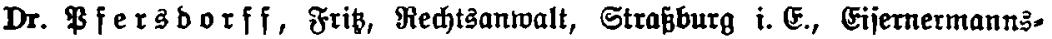
plap 4.

P j i

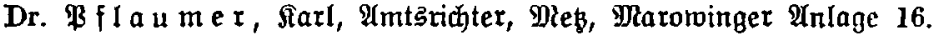

Dr. B f I ü g e r, Brofejfor, Bonn, Saijer Friebridftr. 7.

B f r i e m, Frib, Rechtsantwalt, Bürzurg, Domftr. 11.

Bhil ip $\mathfrak{i}$, Medtsantualt, Meß, Bärenftr. 4.

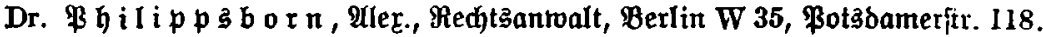

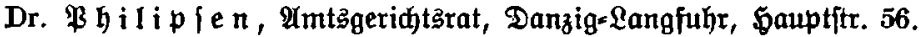

B i đa , Sanbgeriđ)tšrat, Berlin NW 6, Bhilippitr. 20.

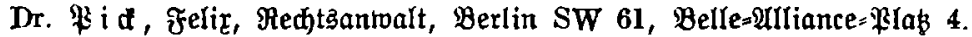

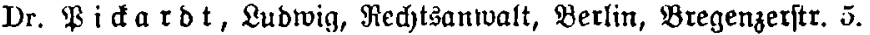

Dr. $\mathfrak{P}$ i g g e , Amtżidhter, Danzig=Langfuhr, Parkneg.

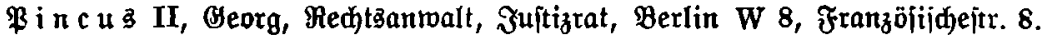

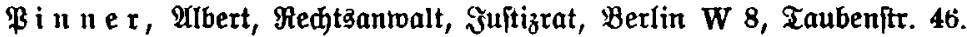

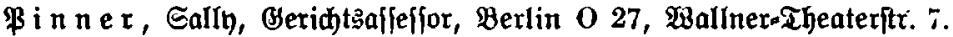

Dr. $\mathfrak{B}$ i p e r, Landgeriđtspräjident, Reujtrelib, Seejtr. 32.

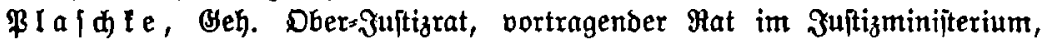
Berlin= Bilmereborf, Mlokfitr. sl.

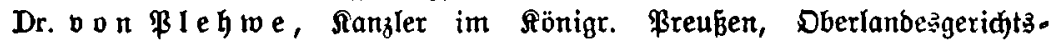

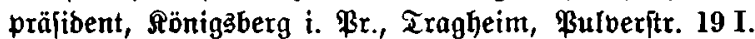

B 1 ○n

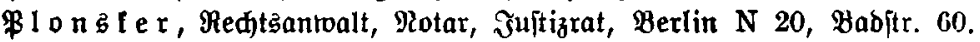

Dr. jur. Rolen ate, Bribatbozent, \$alle a. S., Marlftr. 31.

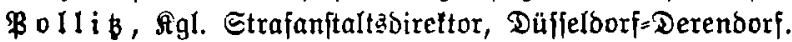

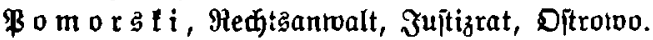

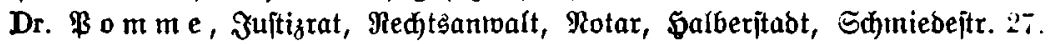

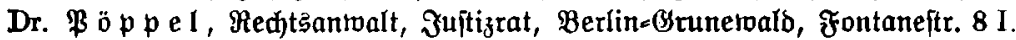

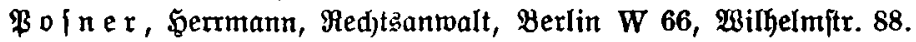

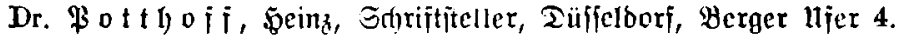


\$r a e I, Mectşantwalt, Бarburg a. E.

Dr. Pralle, R., Medtsantwalt, Motar, Bremen.

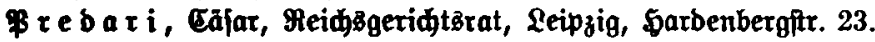

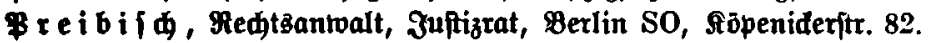

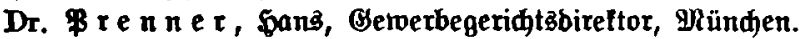

\$ гета и е , Redtzantwalt, Rotar, Suftizrat, Berlin W, Sdellingitr. 5.

Priefter, Garry, Mechtzantwalt, Berlin SW 68, Bimmeritr. 21.

Prinz, beintid, Medtsantwalt, Betlin $W$ 9, Botsbamerftr. 31.

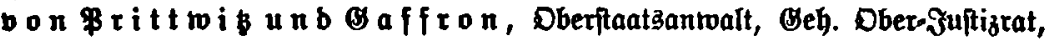
SRaumburg a. S.

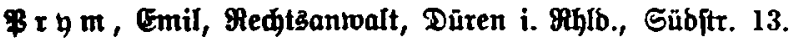

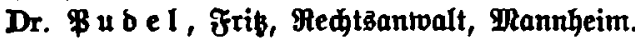

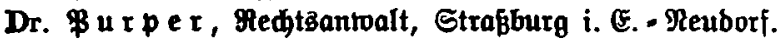

Dr. frabe 16.

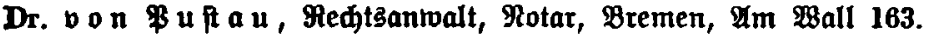

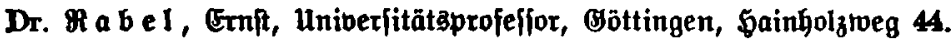

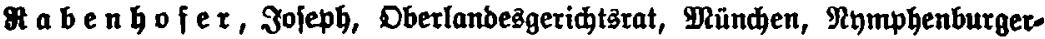
frape 201 .

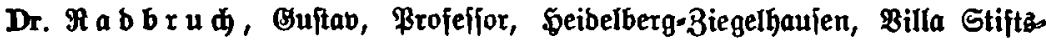
mügle.

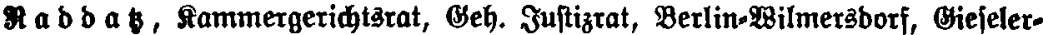
prape 12.

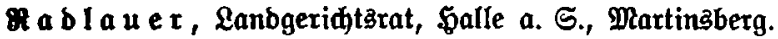

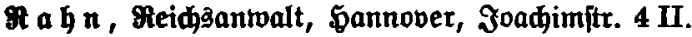

Dr. jur. $\mathfrak{M}$ a f $\mathbf{f}$ e, Marie, Berlin=\&iđterfelbe, Murtenerftr. 4 .

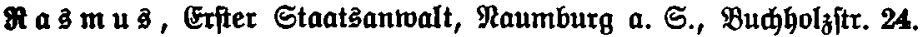

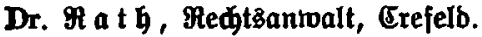

Dr. $\Re \mathfrak{a}$ t $\mathfrak{h}$ e $\mathfrak{a} \mathfrak{u}$, Frib, Regierungąrat, Berlin W 15, Meinefeftr. 7.

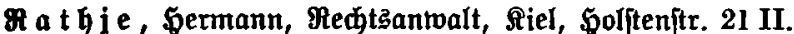

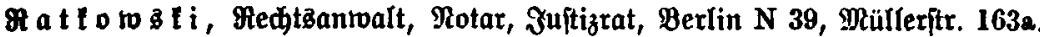

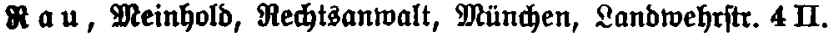

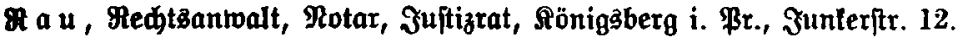

Dr. $\mathfrak{M}$ a $\dot{~}$, Carl, Profeffor, Breslau XVI, Partjtr. 25b.

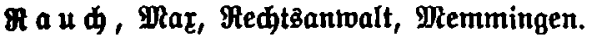

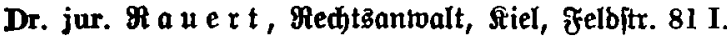

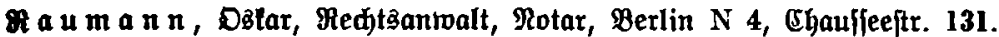

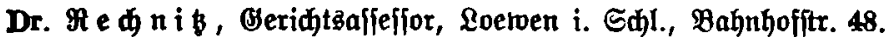

I e đ, Lanbgeriđtżpräfibent, (Beh. Dber $=$ Juftizrat, Seḑingen (5ohenzollern).

Meeb, Redtzanmalt, Stuttgart, Bartenitr. 2.

Dr. jur. $\Re$ e e $\{e$, Riel, Lorenłendamm.

Dr. $\mathscr{A} \in \mathbf{h} \mathrm{m}, \mathfrak{F}$. , Brofeffor, Straßjburg i. (E., \$ohenloheftr. 22.

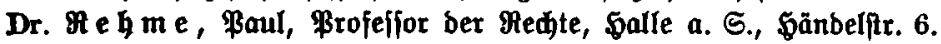

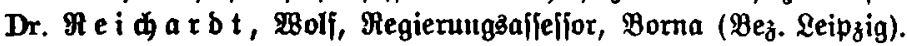

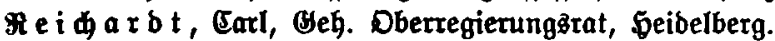

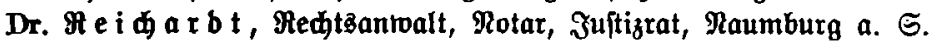

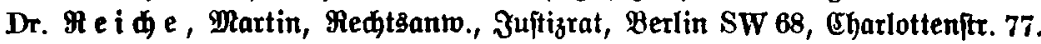




\section{LIII}

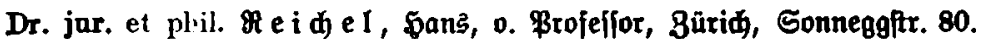

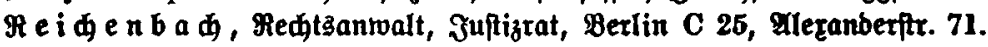

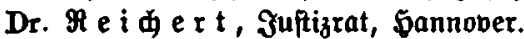

Mei

Dr. M e i na 由, Sultizrat, Mülhaulen i. E., Sinneftr. 16.

Reinberger, Redtsantwalt, Juftizrat, Merlin C 2, Breiteftr. 11.

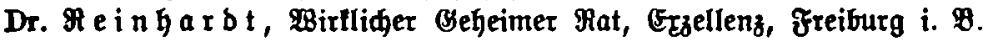

Me in ife, Wugo, Redtsanmalt, Dortmunb.

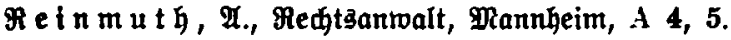

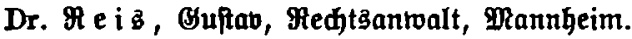

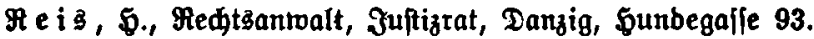

R e i B, Suftizrat, Straß̄burg i. E., Gewerbslaubenftr. 9.

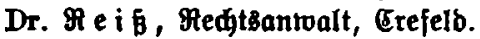

Ie i B, Baul, Juftizrat, Frantfurt a. פR., פejeritr. 51.

Dr. M e i $\mathfrak{n}$ e r, Medtzantwalt, Lanbäberg a. 28 .

M e i B e $t$, Juftizrat, Erfurt, Bartenftraße.

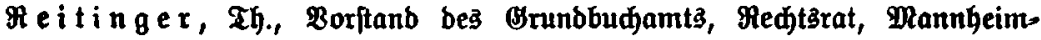
Redarau.

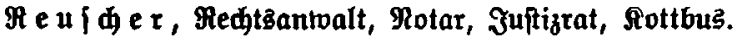

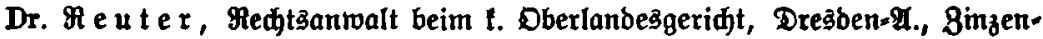
borfitr. 39.

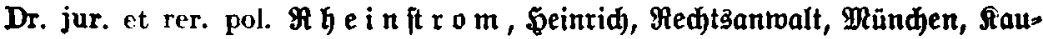
fingeritr. 34.

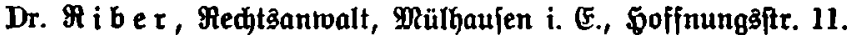

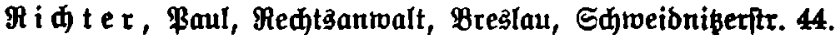

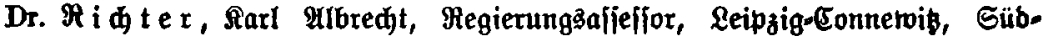
frabe 108.

Dr. $\Re$ i đe $r$, Lanbridter, wieşbaben, Sđübenhofitr. 7.

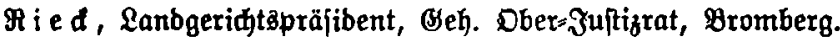

Ri e de, Medtsaniwalt, \$alle a. S.

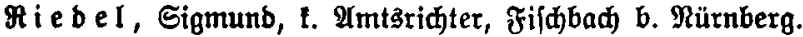

Dr. R i e b e I, Beh. Mat, Raumburg a. S., Budholzft. 41.

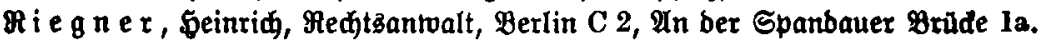

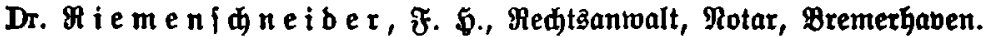

Dr. H i e m \{ d n e i b e r, 2 mtäridter, Bitterjelb.

Dr. Mi efenfels, Conrab Ernft, Syndiłus ber Đanbeläammer, Brofeffor,

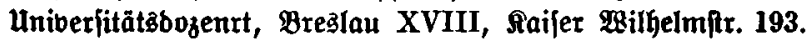

$\Re$ i e B, D., 1. Magiftratzaffeffor, Berlin W 35, Magbeburgerftr. 12.

M i в B, Malter, Med)tzantwalt, Frantfurt a. MR., Reue Mtainzeritr. 35.

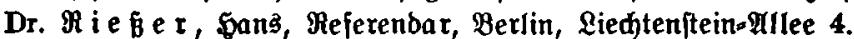

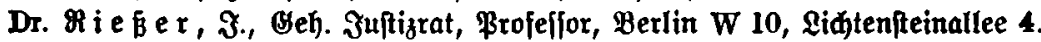

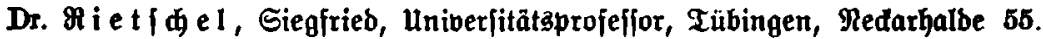

Dr. Mi e $z$ l e r, G., Brofeffor, Freiburg i. O., Reidsgrafenftr. 6.

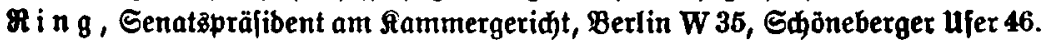

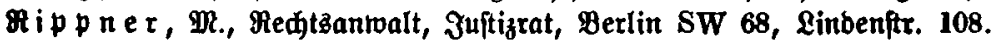

M i $\boldsymbol{B}$, Franz $\mathfrak{X}$., Dberamtäridter, Münden, Breyfingitr. 42.

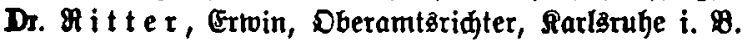


Dr. Fit th a u fe $\pi$, Medtsammalt, Berlin NW 6, \&uifenfr. 39.

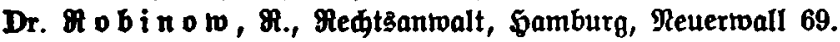

Dr. Roeber, Imtšridter a. D., Direltor bes Rgl. Strafgejängniffe Tegel, Seibelftr. 15.

F o e b i ger, Sermann, Medtsantwalt, Mannheim L 4, 1 .

P off $\mathfrak{h}$ a d, Senatsignbihus, Bremen, Sutfilterftr. $2 / 4$ III.

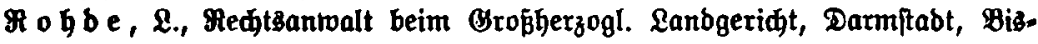
maxdfraje 15.

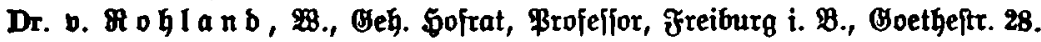

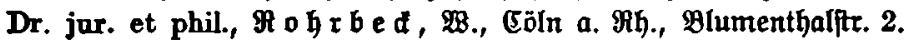

M ó hi g, Medtzantwalt, Berlin NW 7, Mittelftr. 63.

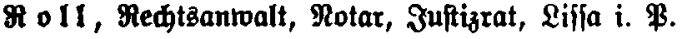

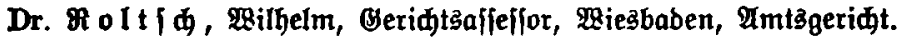

Dr. Mo m e i

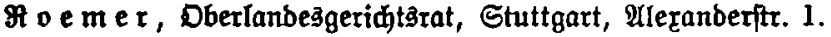

Dr. M o m e r, Međtsantwalt, 9lotar, Jujtizrat, Bodum i. 28.

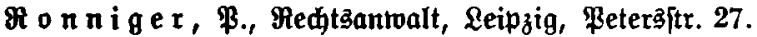

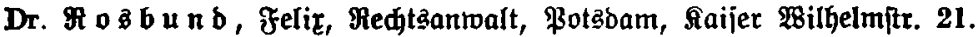

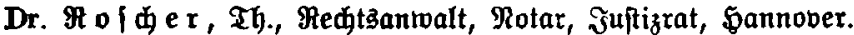

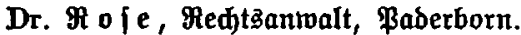

$\Re$ ofe, E., Medtzantwalt, Notar, ફarburg a. E.

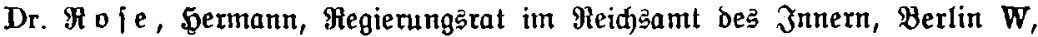
2qilhelmitr. 74 .

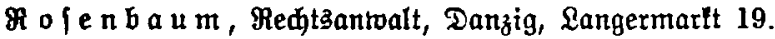

Dr. M o f e $\boldsymbol{b}$ er

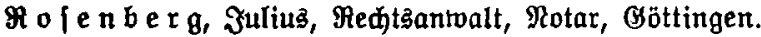

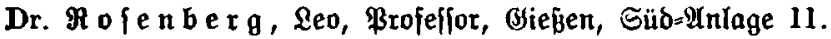

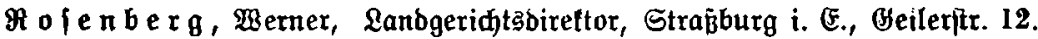

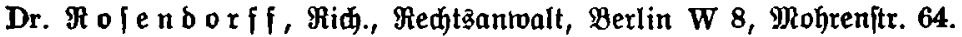

Dr. jur. et phil. $\Re$ o fe $n f e l d$, Ernit, Staatsantwalt, Berlin W9, Bopjitr. 13.

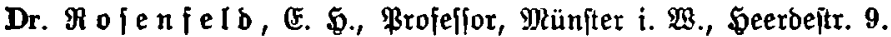

Dr. $\mathfrak{R}$ o f e $n$ f e I $\delta, \mathfrak{F} .$, Redhtzanivalt, Mamneim.

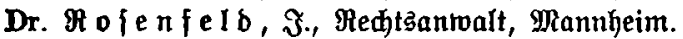

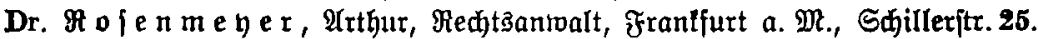

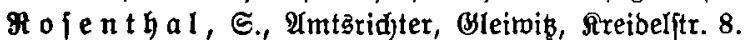

F o le $n \in \mathfrak{h}$ a l, Landgeridtabireltor, (Seh. Juftizrat, Danzig, Borftabtgraben la.

$\Re$ of e $\mathfrak{n} \mathfrak{t} \mathfrak{h} 1$, Landgeridgtsbireltor, Charlottenburg, Sdhlüteritr. 63.

$\Re$ olenthal, Malter, Medtsantwalt, Berlin W 35, Benthinerftr. 58.

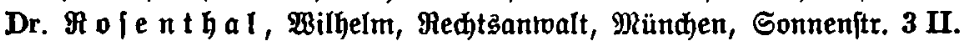

Dr. Mofi n, Beh. Mat, Brofelfor, Freiburg i. $\mathfrak{B}$., Büntherşthal 64.

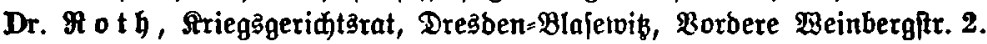

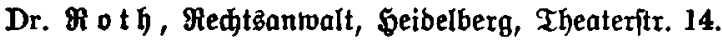

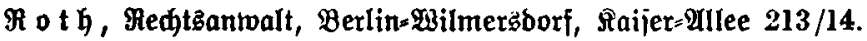

Dr. Bothe, Juftizrat, Berlin C 2, Raifer Billhelmftr. 39.

Rothe, E., Redtsanwalt, Mitenburg $\mathcal{S}_{.=} \mathfrak{A}$.

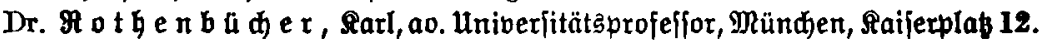

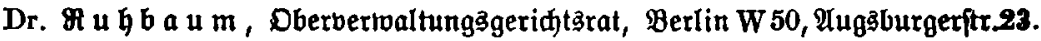




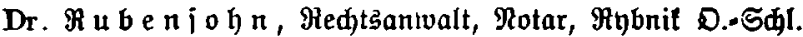

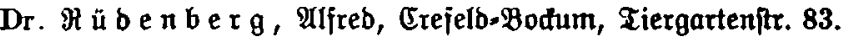

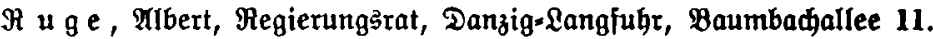

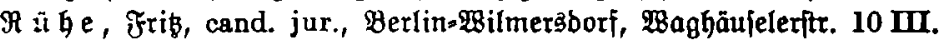

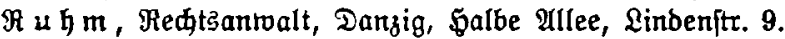

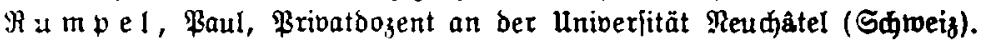

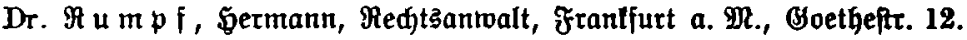

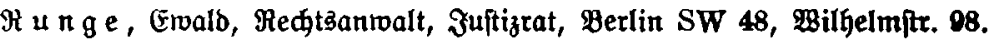

Dr. v o n $\mathfrak{R}$ üp $\mathfrak{p}$ i $\mathfrak{n}$, Freiherr, Randgeridtsbireltor, Ronitanz.

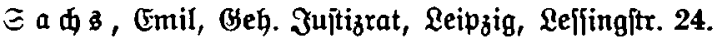

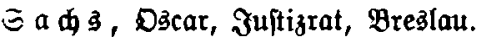

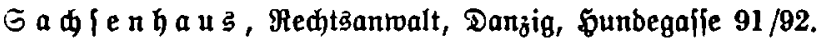

Dr. jur. Sad, Th., Sdriftiteller, Mlünden, Franz Jofephitr. 20.

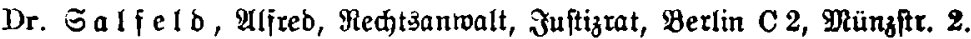

Dr. Galf eld , Afjed, Med)täantwalt, 3uptizrat, Berlin C 25, Mranzltr. 2.

G a li in g e r, Mlax, Medtsanwalt, Rotar, Juptizrat, Berlin W 10, van ber Geybtftrafe 3.

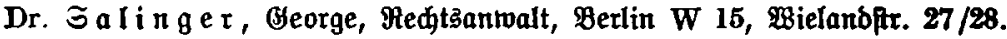

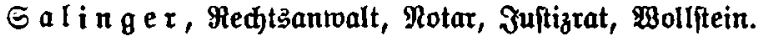

Dr. Salinger, Dberlanbeşgeriđtşrat, Bres̆lau, Gohenzollernitr. 28.

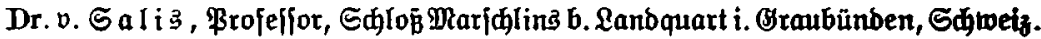

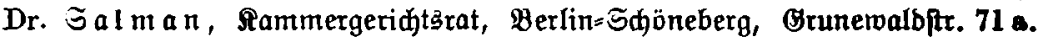

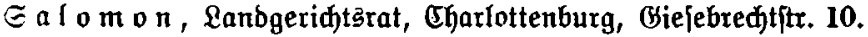

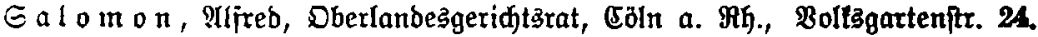

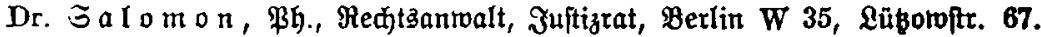

Sal o m on, $\mathfrak{s}$., Red

Dr. ङ a 1 o m o n, Reopold, Dreşben, Maridjallitr. 55 I.

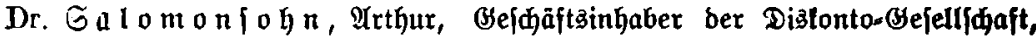
Berlin W 64, Unter ben Sinben 35.

E a mo ie, f̧erbinand, Medtsantwalt am Sammergeridt, Berlin W 35, Botzbamerftráje $39 a$.

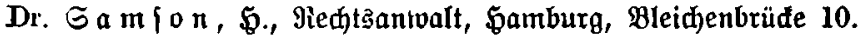

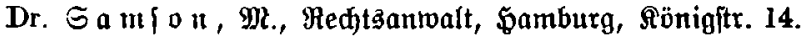

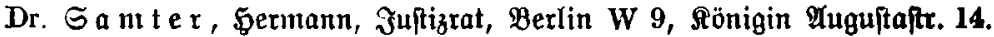

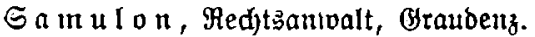

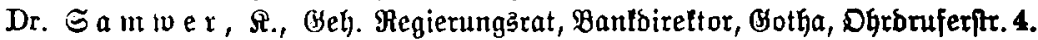

Ga n $b$ berg, Buftav, Medhtsantwalt, Juftizrat, Berlin $S$ 42, Rottbuferptr. 6.

Dr. Sander, E., Redtalantwalt, Berlin W 57, Elaholzitr. 2.

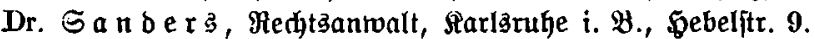

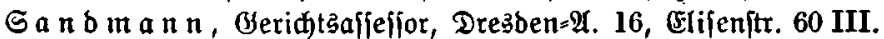

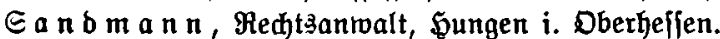

હä $\mathfrak{n} \mathfrak{g} \mathfrak{e} \mathbf{r}, \mathfrak{A} .$, Redtsantwalt, Stuttgart.

Dr. Sart oriu 3 , Brofeijor, Tübingen.

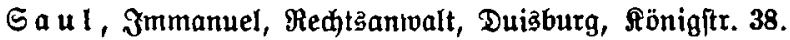

ङ凹

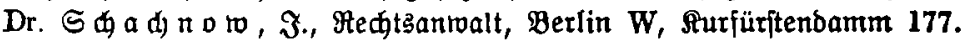

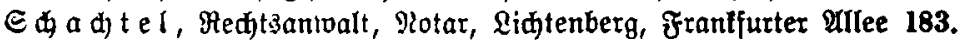


S申 a e fer, Medtzantwalt, Breslau V, Tauenjienftr. 2.

Dr. S 由 a effer, Staatbantwalt, Oleitwil 0. Shl.

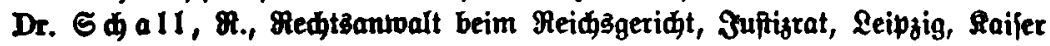
Milfelmftr. 12b.

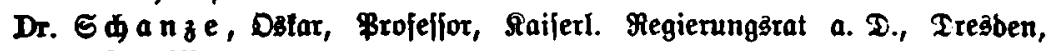
Sacobiftr. 5.

Dr. S由 a red, Geridt taffeffor, Matibor.

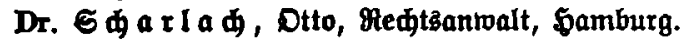

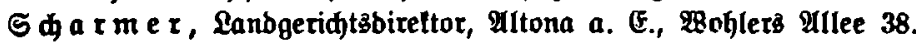

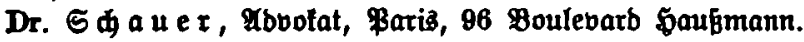

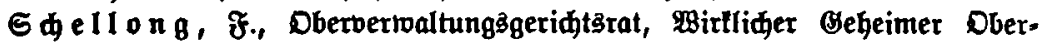
regierungsrat, Charlottenburg, Carmeritr. 10.

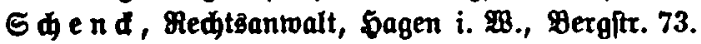

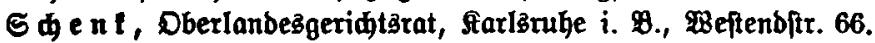

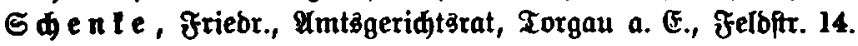

S申epers, Lanbgeridtarat, Raberborn.

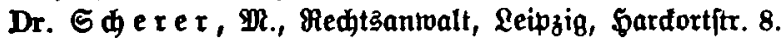

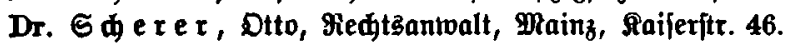

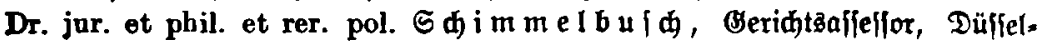
borf, Bubensftr. 4.

ธ中i

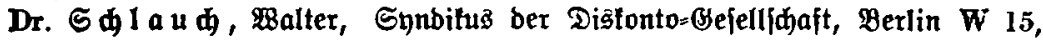
Dieineteftr. 2.

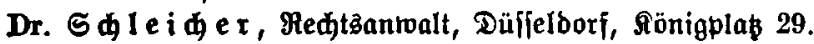

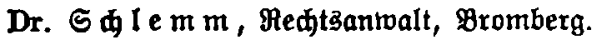

Sqlefinger, Beorg, Medtaninalt, Leipzig, Reumartt 1.

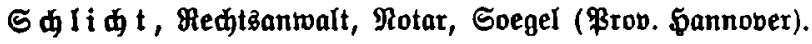

Dr. S由li et e $r$, Redtŝantralt, Bromberg.

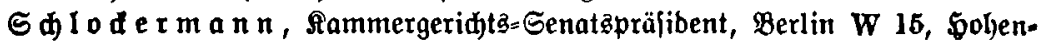
zollernoamm 5.

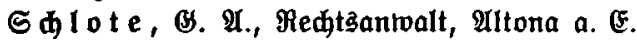

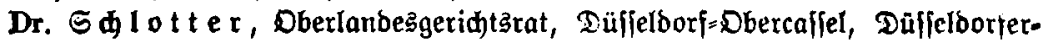
ftraje 25.

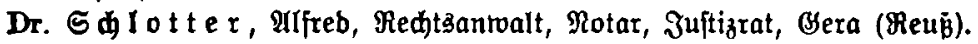

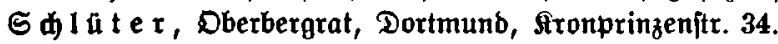

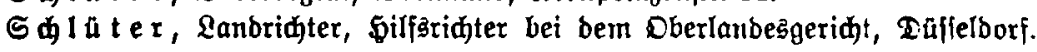

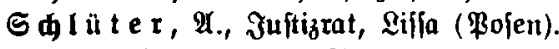

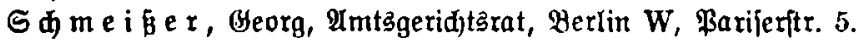

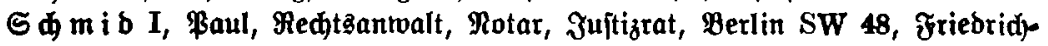
firafe 248.

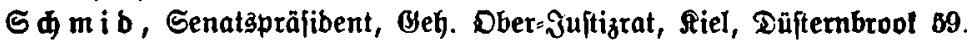

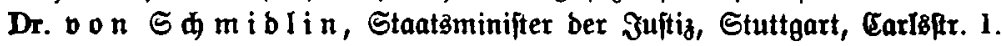

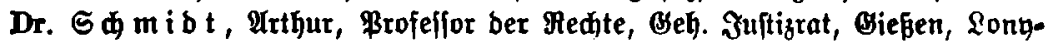
frape 18.

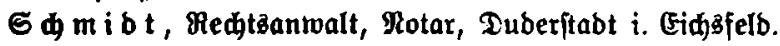

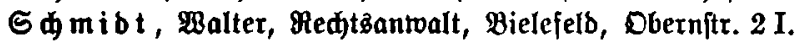

Dr. 5 d m i b t, Midarb, Beh. Sofrat, Brofefior, Freiburg i. $\mathfrak{B}$. 


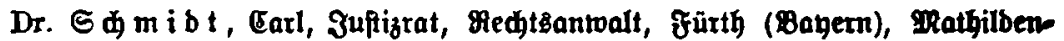
frafie $10 \mathrm{p}$.

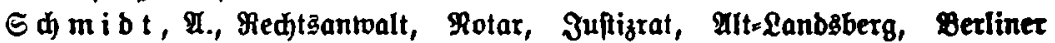
allee 13.

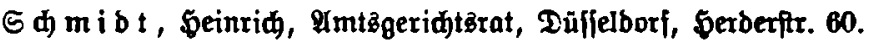

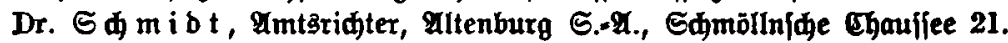

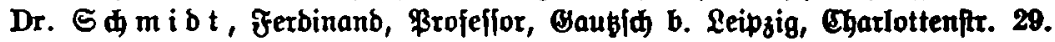

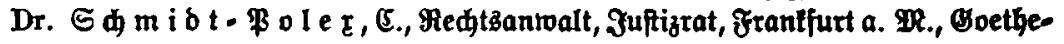
plaßs 16.

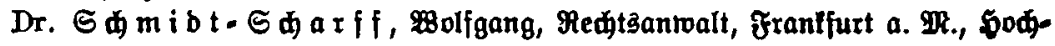
frape 10.

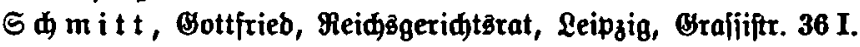

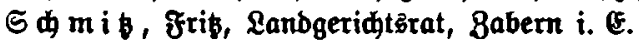

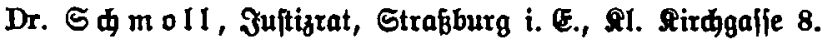

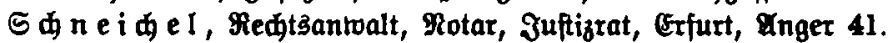

Dr. S 4 n e i b e r, Otto, Lanbgeridtsrat a. D., Bantbirettor, Mannbeim A 2, 1.

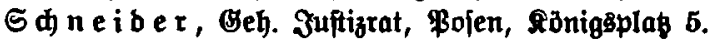

Dr. S中ne i ber, Bittor, Mectsantwalt, Suftizrat, Berlin W 64, unter ben Linben 11.

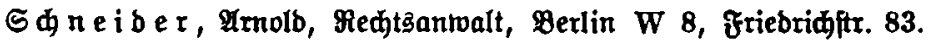

S q ne i b e $r$, Johannes, Medtsantwalt, Rotar, Meerane i. E.

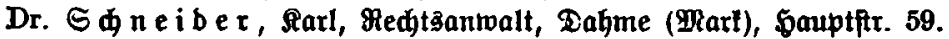

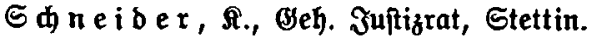

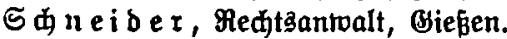

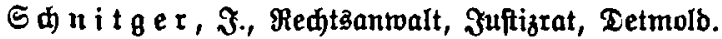

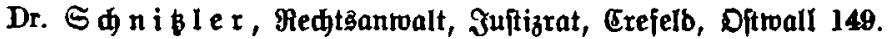

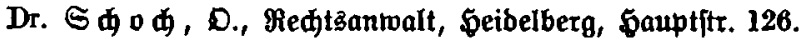

Dr. S๙ o e mann, Rectsanwalt, Rgl. Bergiwertabirettor a. D., Bleimib̧, Bromenabe 5.

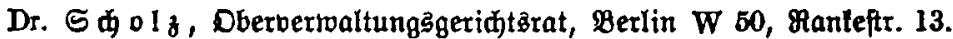

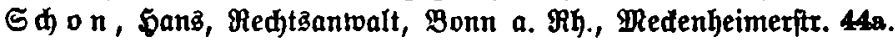

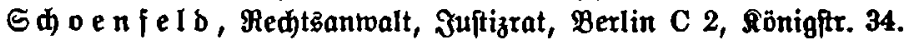

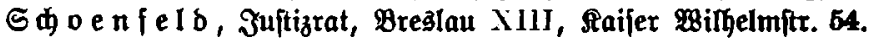

Dr. Sdoenborn, 2B., Privatbozent ber Medte, Seibelberg, Bähringer itrake 16.

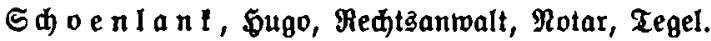

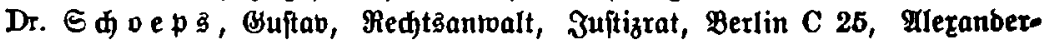
ftrake 53.

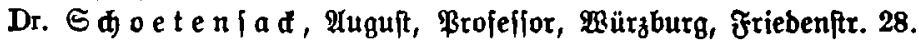

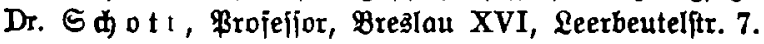

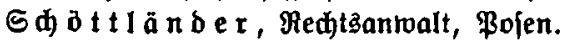

Sd) $\mathbf{a}$ ber, R., Eijenbahnbirełtor a. D., Berlin W 35, Stegliferftr. 68.

ธ๙ $\mathbf{r}$ a ber,

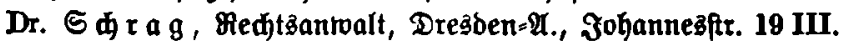

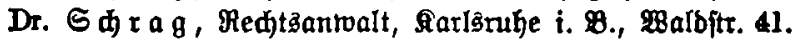

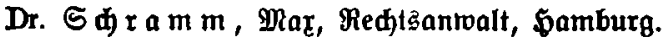

S出 $\mathbf{e}$ i b e $r$, 2uguft, Redtsanmalt, Donauejdingen. 
Gdreier, Datar, Medtantwalt, Berlin C 2, Reue \$romenabe 3.

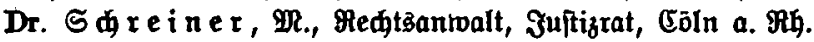

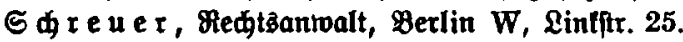

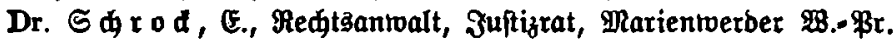

S d r o e l e r, Earl, Amt3ridter, Birnbaum (\$ojen).

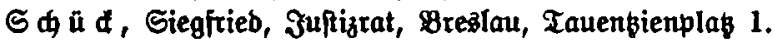

S 中 ũ le r, Buftav, Medtsanmalt, Stolp i. \$.

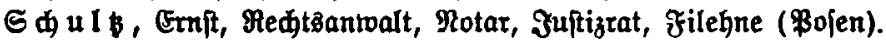

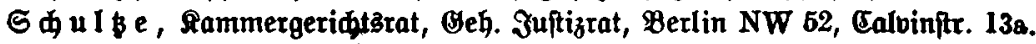

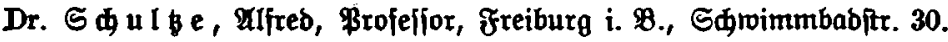

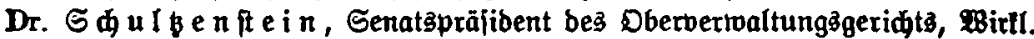

Beh. Dberregienungärat, Berlin W 30, Mlob̧itr. 86.

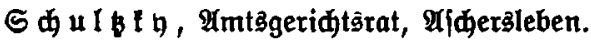

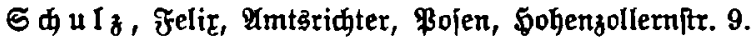

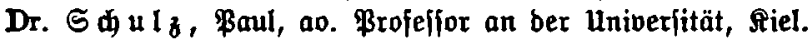

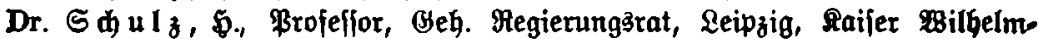
frape 33.

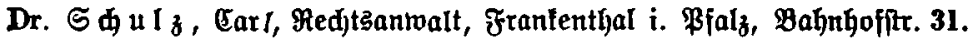

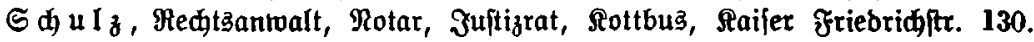

von $\subseteq$ d $\mathfrak{u} I_{z}$, Magiftatzrat, Berlin SW, Bimmeritr. 90/91.

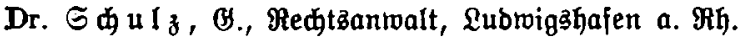

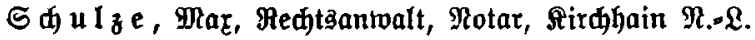

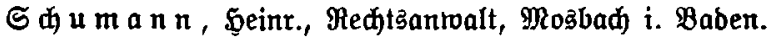

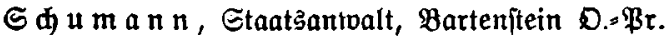

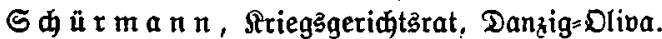

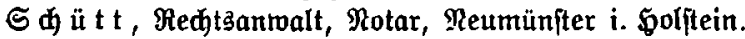

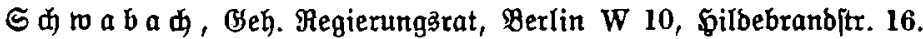

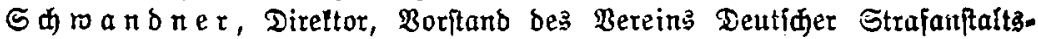
beamten, Ruowigsburg i. $23 t t b g$.

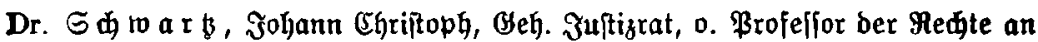

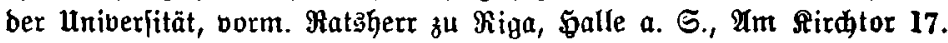

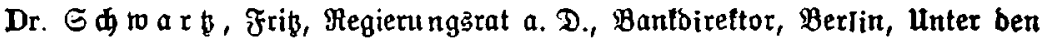
Linben 34.

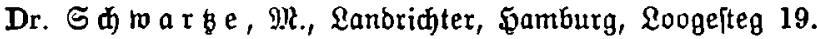

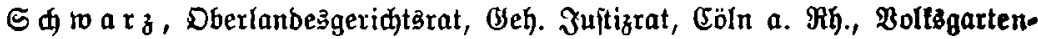
ftrabe 23.

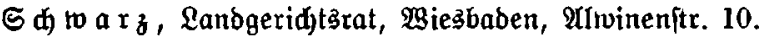

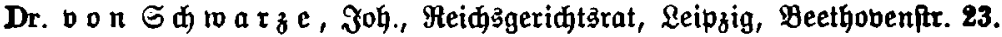

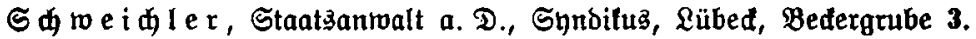

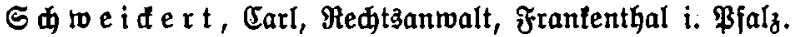

Dr. Sd wei gh of fer, Megierungarat a. D., Berlin W 9, Lintitr. 25.

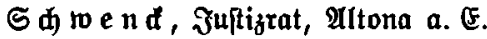

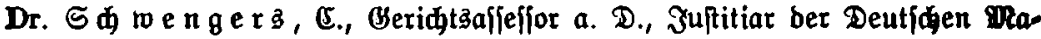
\{dinenjabrit, $\mathfrak{A}$. B., Duisburg.

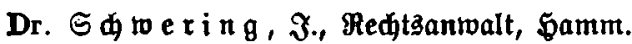

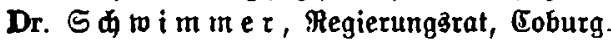


Ecriba, 5 ., Syndifua ber Lanbro.-Pammern für bie \$rovinz Sadjen, Đalle a. S., Ulejtr. 8.

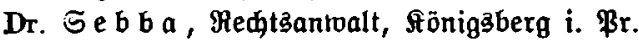

Dr. Se đel, E., Beh. Juftizrat, Profeffor, Oharlottenburg, WBiglebenplaß 3.

Dr. Gée, Éggarb, Mboolat, Garis, 4 rue le Peletier.

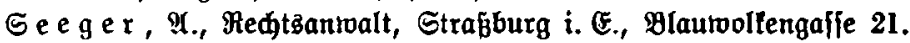

Dr. v. Se e I e r, $\mathfrak{B i l h}$., ßrojeffor, Dorpat Univeriität.

Dr. Seelig, Redtżanmalt, Berlin W 9, Botżbameritr. 9.

Dr. Se el i g, Lubrig, Redtäantwalt, Mannheim.

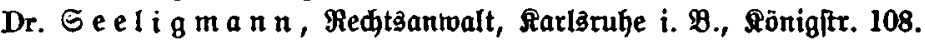

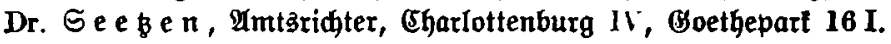

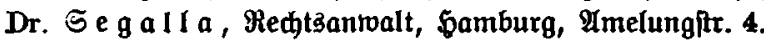

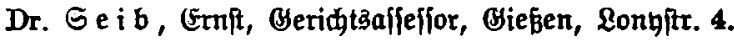

S e i b e t , Ridter, Bremen, Bismarditr. 71.

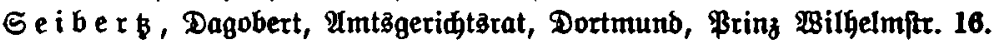

Dr. Se il er, Dtto, Redtsanwalt, Mannbeim.

S elb, G., Fistalantwalt, Mannheim, A. 3, 9.

S el d, Ermin, Reфtsantwalt, Frantfurt a. Mt., Feuerbadftr. 50.

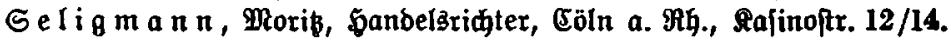

Dr. Seligiohn I, Arnolb, Jujizizat, Redtzantwalt, Berlin NW 7, Prinz Qouis Ferbinanbitr. 1.

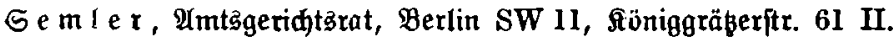

Senif, Hedtzanivalt, Juftizrat, Berlin W 8, Taubenitr. 44/45.

Ge n g e r, Mar, Redtłanivalt, Juitizrat, Braubenz, Amtzitr. 19.

S e n n in ge r, łofef, Rechtsantwalt, Baf\{au, Rubrigitr. 285.

Dr. Seybel, Đalle a. S., Aleine Steinftr. 1.

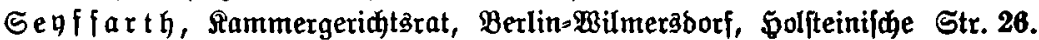

Dr. Seyfart, Redtåanwalt, Breiz, Martt 14.

Dr. Geyfert, Eurt, Redtananivalt, Seipzig, Bismarditr. 8111.

Seyfert, F. S., Senatspräjibent, Dresben, Stephanienfitr. 30.

Dr. $S_{i} \mathfrak{d} \mathfrak{e l}, \mathfrak{J u f t i z r a t , ~ M a i n z , ~ B a u h o f i t r . ~} 4$ I.

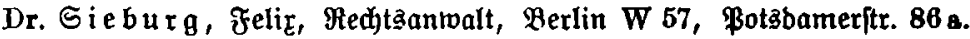

Dr. S i e gel I, Leopolb, Reфtsantwalt, Juftizrat, Münфen, Dienerftr. 20.

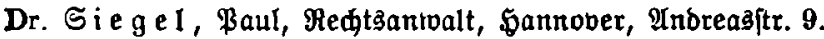

Dr. Siegcr, F., Redtsanivalt, Mtotar, Juftizrat, frantfurt a. Mr.

Dr. Sieg ma nn, Beorg, Medtsaniwalt, Berlin SW 68, Linbenftr. 112.

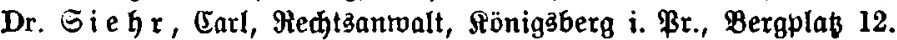

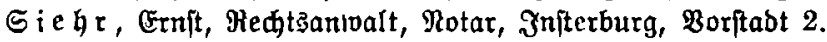

Dr. Sie $\mathbf{t} \mathfrak{i}$ ind, Randridter a. D., Bieß̧baben, Raijergof.

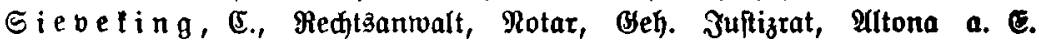
Bahnhofitr. 20.

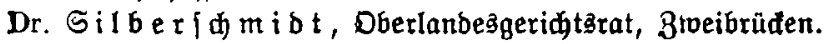

Dr. S i l b e r ft e i n, Mar, Redtsanivalt, Juftizrat, Berlin W 10, Margaretenfrape 7 .

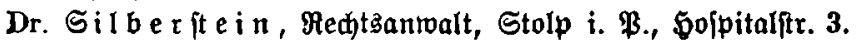

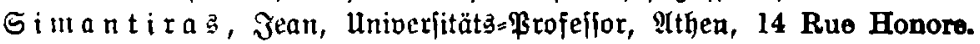

Dr. Si m é on, $\mathfrak{B}$., Stammergeriđtşrat, Berlin W 15, 9regenzerftr. 5. 


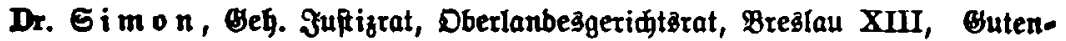
bergitraje 47.

Dr. Simon, Medtzantwalt, Meiningen $\mathcal{S}_{.}=\mathbb{M}$.

Dr. Sim on, Emil, Medtannalt, Düffelborf.

Dr. Si m o n, 5. 8., Pedtzantwalt, Suftizrat, Berlin W 10, Bittoriaft. 5.

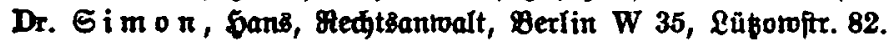

Dr. Si m o ni B, Moolf, Purgermeifter, Moftod i. Medl., St. Beorgltr. 28.

S i m o n $8,28 .$, Lanbridter, Bexlin-Qidterfelbe, Stegliberftr. 39.

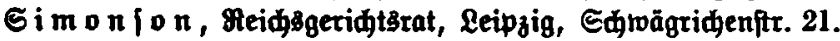

o on Sim fon, Redttanmalt, Motax, (Seh. Juftizrat, Berlin W 56, Jăgerfrape 62.

S in $\pi$ P., Medtsantwalt, Rotar, Juftiztat, Renbsburg.

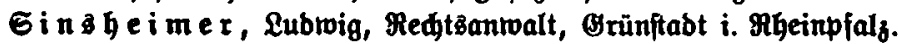

Dr. S i n z

Dr. Si q u e $t$, Medtæantwalt, Straßburg i. E., Manteuffelftr. 35.

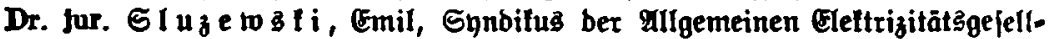

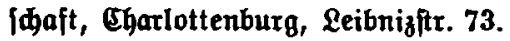

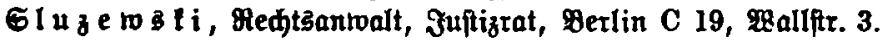

Dr. S m o i $a$, Mr., Feferenbar, \$önigäberg i. \$r., Maijerftr. 49.

Gmolla, Beorg, Rotar, Breslau V, Tauentienftr. 15.

Dr. Soberngeim, Otto, Beridtąaffefjor, Berlin NW 40, Alfenftr. 3a

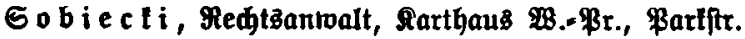

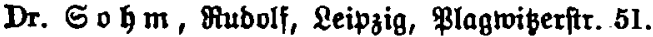

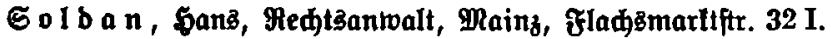

Dr. Solmfle n, Beorg, Bejđäftainjaber b. Distonto-Befell/daft, Berlin W64, Unter ben Linden 35.

6 o m mer, OAmtżridter, Reibenburg i. \$r.

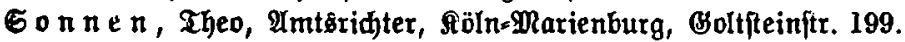

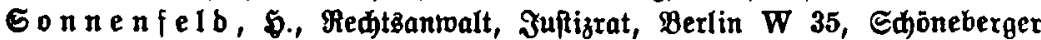
ufer 22.

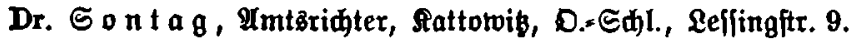

Dr. S o e t b e r, Beneraljetretär bes Deutj內en Đanbelstages, 2 annjee, Sohengrinftrafe 28.

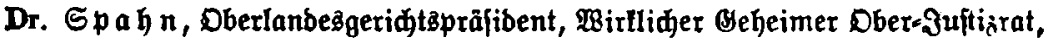
Franlfurt a. $\mathfrak{Q R}$.

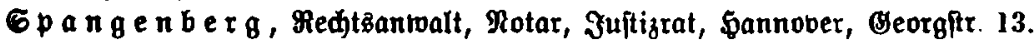

Dr. Jur. Sperling, Seinrid, Lanbgeridtłprälibent, \&nd $D .=\mathfrak{B r}$.

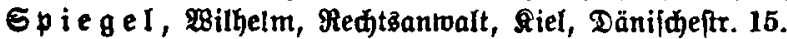

Dr. Sp ittel, Ređtzantoalt, Rotar, Rottbus, Raijer friebridjttr. 126.

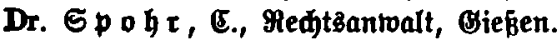

Gp ringe, Berner, Medtâantoalt, MItona a. E., Große Bergftraße.

Gp ringer, Frib, Meferenbar, Oharlottenburg, 巨alluterftr. 45.

Dr. Springer, Ernfi, Juftizrat, Berlin W 9, Lintft. 23/24.

Gt a e b e I, E., Ftedtsanwalt, Darmitabt, E్lifabethenftr. 14.

Dr. Sta bthagen, Jul., Motar, Juftizrat, Perlin SW 68, Bimmerfir. 19.

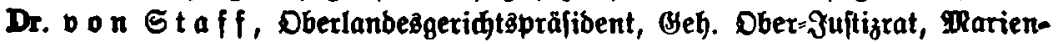
werber $28 .-\$ 3$ r. 


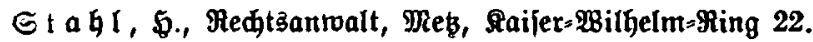

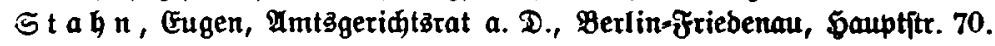

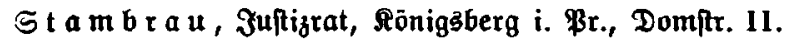

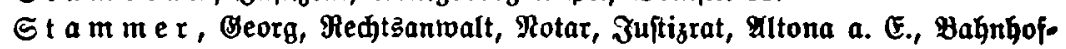
ptrake 46.

Dr. St a m mler, Brofefior, (Jeh. Juftizrat, Đalle a. G., Meidarbtitr. 13.

St ap $\{f, \mathfrak{A} .$, Mectsantwalt, Juftizrat, Meimar.

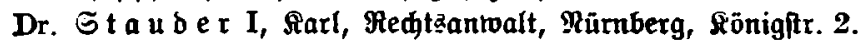

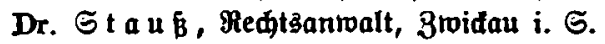

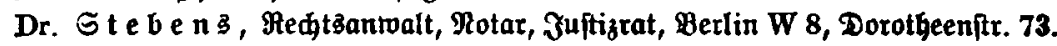

Dr. St e in, Friebrid, ßrofelfor, Leipzig, Ferbinand Mhobeftr. 28.

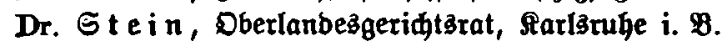

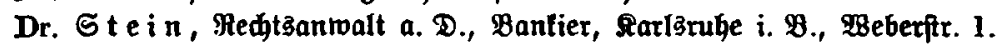

Dr. St e in, Suftizrat, Thorn.

St e i nel, Eugen, Fedtsantwalt, Bjorzheim.

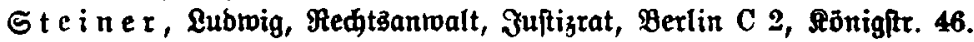

Ete iner, Medtzanmalt, Matibor.

St e iner, B., Redtsanmalt, Colmar i. E.

Dr. St e in f el b, DlaE, Suftizrat, Breslau, bohenzollernipr. 79.

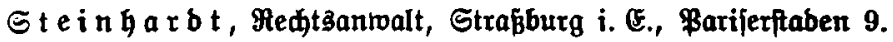

Dr. Ste ini

Dr. Ste inis, Mar, Reळtżanwalt, Berlin N 54, Brumenftr. 25.

Dr. St eini

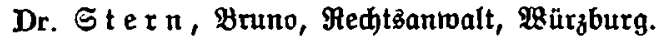

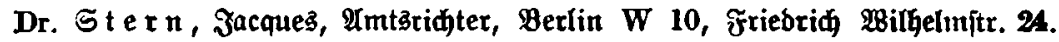

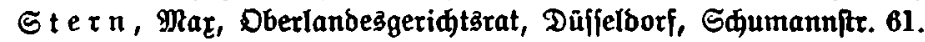

Dr. St ex $\pi$, Otto, Eandridter, Beuthen D.-Sdjl., Bhmnafialikr. $15 a$.

Dr. Єtern, Fedor, Medtşantwalt, Rotar, 3uitizrat, Berlin W 8, Begrenitrafie 23.

Dr. Ste $x$, S., Redtảantoalt, Mannheim.

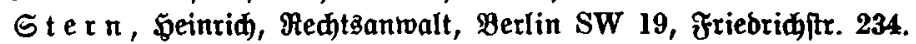

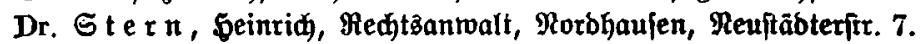

Ster $\pi$, Rubrig, Lanbgeridt3rat, Stuttgart, Seeitr. 7 .

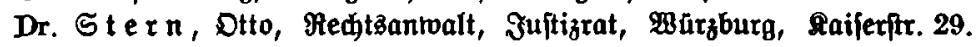

St e rnber $B$, Lanbgeriđtärat, Eharlottenburg, Brolmanftr. 6.

Sternfelb, Medtsanivalt, Danzig, Reitbahn 23.

Dr. G te t te nhe i mer, Redtzantwalt, Frantfurt a. MR., Beethmannft. 50.

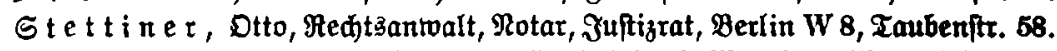

Et e yrer, Frib, Direltor ber Bayr. Đandelabant, Münden, Maria Dherejiafrape 13.

Dr. Sti ef, Lanbridter, wiesbaben, An ber Ringlirde 7 .

Dr. St i e - So m I o, Uniberfitätäprofeffor, Bonn, Raufmannitr. 81a.

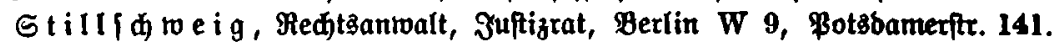

Dr. Sti $\mathfrak{n}$ i $\mathfrak{g}, \mathfrak{B}$., Profeffor, Leipzig, Sibonienftr. 67.

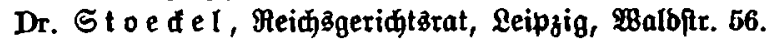

Dr. St ol ze, Ridarb, Magiftat3affeffor, (5̧arlottenburg, Winbldeibitr. 34.

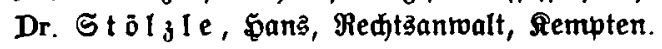


G t oxy, Mfreb, Mechtşanwalt, Bexlin W 8, Taubenftr. $16 / 18$.

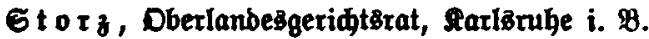

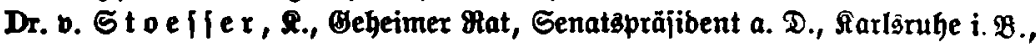
Stephanienftr. 88.

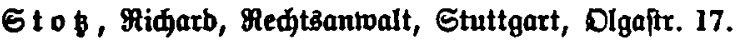

Gtrae hler, Pammergeridtbrat, Berlin W 50, Beiabergitr. 12/13.

Dr. Stranz, MR., Rectsantwalt, Berlin W 62, Яurfürftenitr. 73.

Straffer, f., Lanbgeridtżat, Bayreuth.

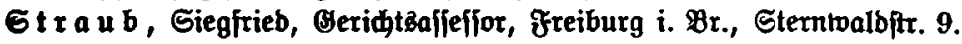

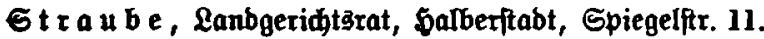

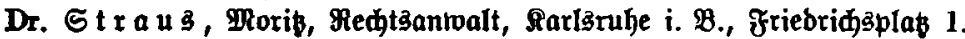

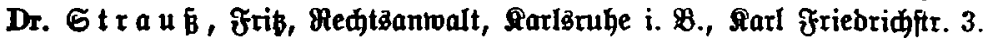

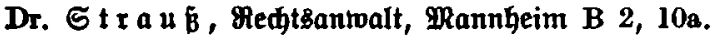

Et refler, \&ubrig, Redtizanwalt, Dieb, Ponceletftr. 2.

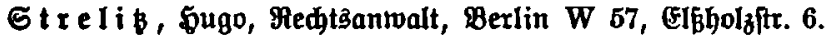

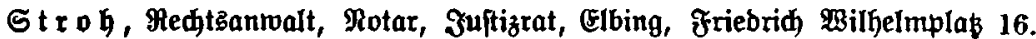

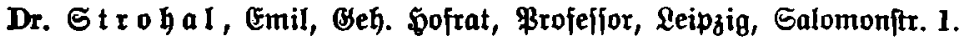

Dr. St $\mathfrak{x} \mathfrak{u} \mathfrak{m} a \mathfrak{n} n$, Buptav, Beh. Regienungšat, Berlin W 30, Rojen= heimerftr. 24.

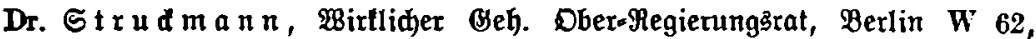
Lanbgrajenitr. 15.

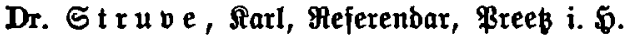

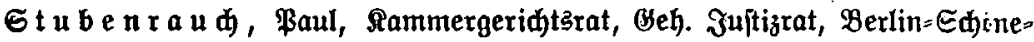
betg, Mobjitr. 56.

Dr. St ūb be n, Regierungsalffeffor, Bojen, Bor bem Berliner Tor 3.

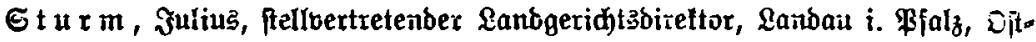
bahnfráze 18.

ङ $t$ u $t$ t e, Erid, Bseridtaffeffor, Erejelb, weftwall 209.

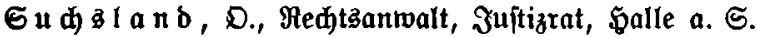

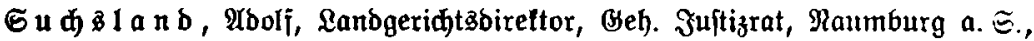
Bưhyolzitr. 30.

$\mathfrak{S} \mathfrak{u} \mathfrak{a} \mathfrak{a} \mathfrak{u}, \mathfrak{s u f t i z r a t , ~ D a n z i g . ~}$

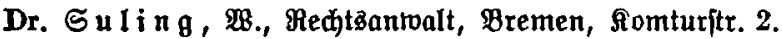

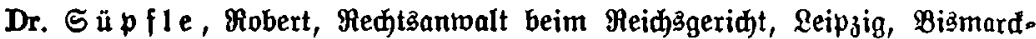
ftraß̄e 12.

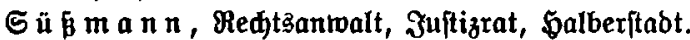

Gupper, Generalitaatąantwalt, Berlin W 30, Landshuterftr. 23 I.

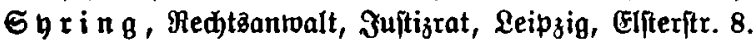

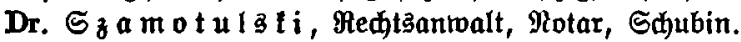

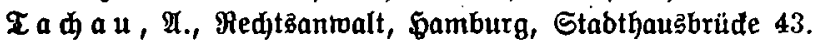

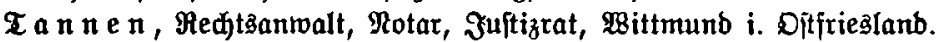

Dr. Ia e nzler, Frib, Synbifus ber \$auptitelle ber beut\{dyen Irrbeitgeberverbänbe, Berlin W 9, gintftr. 25.

Tecoro, Mlifed, Magiftratšrat, Berlin W 10, Ban ber Gendtitr. 7.

Tei $\mathbf{m} a \mathfrak{n} n$, Eandgeridtsrat, Stuttgart, Mozartfr. 20.

Dr. Tentler, Redtzantaalt, \$amburg, Stabthauıbrüđe 43.

T e to a $a$, Beh. Juftizrat, Dortmuno. 


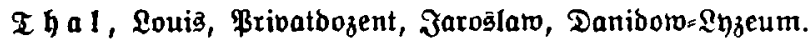

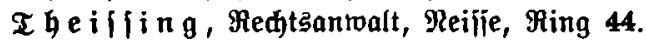

I hie 1 , Jutizrat, $\mathfrak{B r e j}$ )en.

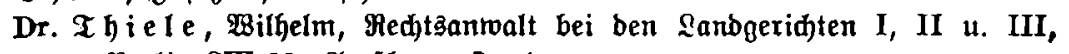
Berlin SW 11, Grobbeerenitr. 4.

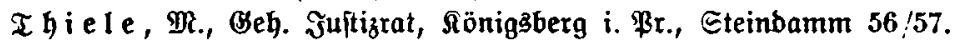

Th i e ma n $\mathfrak{n}$, Max, Međtzarwalt, Braunjdreig, Bohlweg 66.

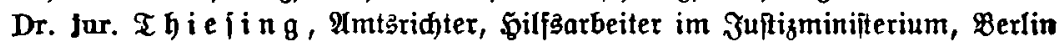
W 30, פeilbronneritr. 16 III.

Thi niu Mohrenitr. 65.

Dr. $\mathfrak{T} \mathfrak{h} \cup \mathfrak{m a}, \mathfrak{u n i v e r [ i t a ̈ t a p r o j e f f o r , ~ G e i b e l b e r g . ~}$

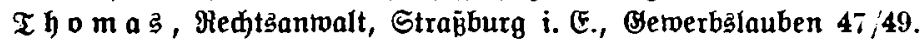

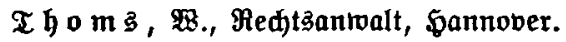

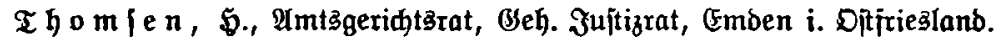

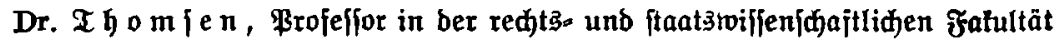
ber Univerfität Münfter i. B., Georgžcommenbe 1 .

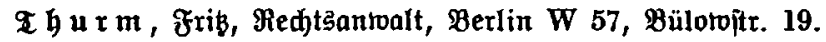

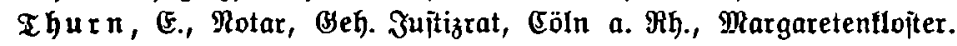

Tille fe $n$, Redtsanwalt, Mannheim.

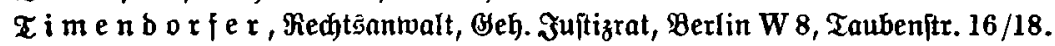

Dr. T i \& e, Brofefjor, Direftor bes juriftifden Seminara, Bsöttingen.

Dr. I i ze, ßrofeffor, Büttingen, Dbere faripule 35.

Tollfie mit, Redtłanroalt, Geheimer \}uftizrat, Raumburg a. S.

Dr. To e pli b, Seon, Jujtizrat, Dreảben, Maridallftr. 1 WI.

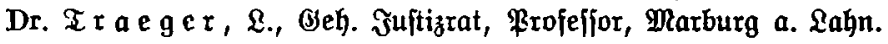

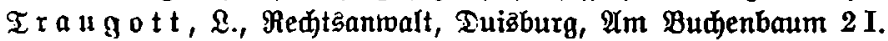

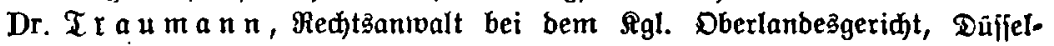
borf, Bartenftr. 25.

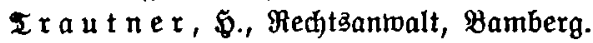

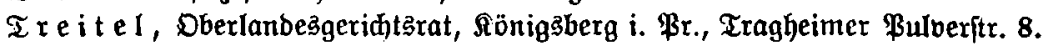

Dr. Tra mpler, Gynbilus ber Sandeläammer, Frantfurt a. Mi., Börje.

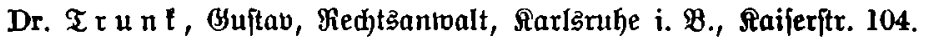

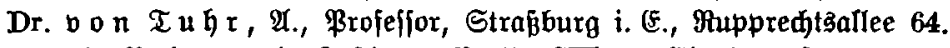

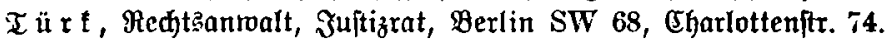

$\mathfrak{u} f \mathfrak{l}$ a d e $r$, Juftizrat, Ultona a. E.

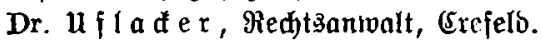

$\mathfrak{u} \mathfrak{h}$ les, Emil, Beh. Suftizrat, Rammergerid)tşat, Berlin W 10, Tiergarten. jtraje $3 a$.

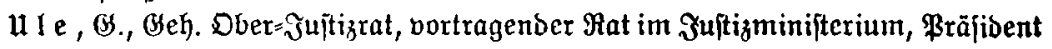

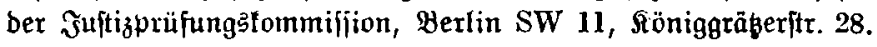

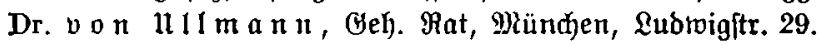

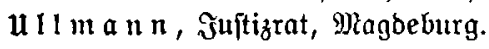

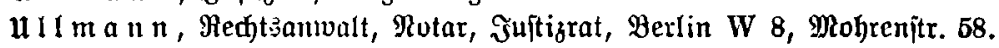

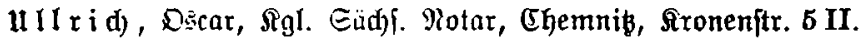

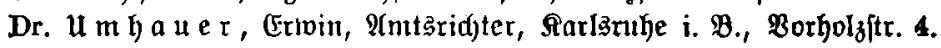

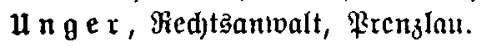




\section{LXIV}

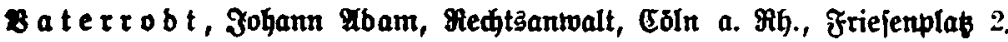

Bi e bi g, E., Jultizrat, Betlin W 67, Balowitr. 24.

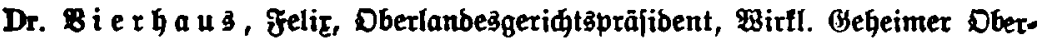

Suftizrat, Brofeffor, BresIau XIII, Sokenzoflernitr. 95/97.

B l e u g e Is, Rotar, Juftizrat, Eoln a. Mh., St. Apernftr. 17.

Dr. Bogel, Carl, Behtzantwalt, Maftatt.

Dr. 8 o g e I, Meferenbar, Blajerwił b. Dresben, Berggartenitr. 9 II.

$8 \delta$ g e l e, S., Redtantwalt, Rarlaruhe i. $\mathscr{B}$.

B ० g I , Carl, Lanbgeridtsbizettor, Eraunftein i. 8 .

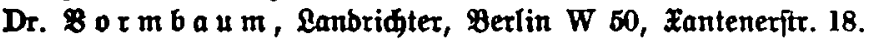

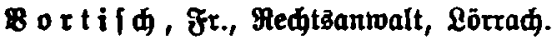

$B \circ$ B, Juftizrat, Dftrowo.

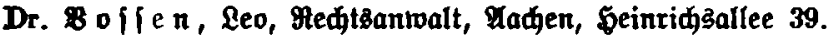

Dr. $\mathfrak{B}$ 出, Birflider Bebeimer Mat, Erzellenz, Brofeifor, Leipzig, Bartftr. 11.

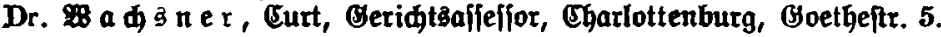

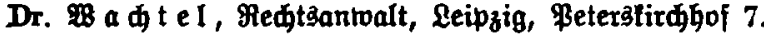

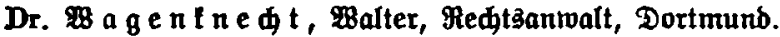

Wa gn e r, Бanns, Redtzantwalt, Lubrigh̆afen a. Mh., Anilinfabrif.

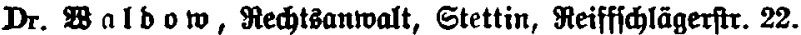

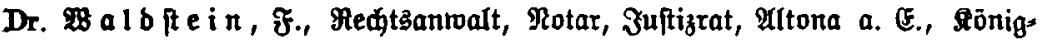
ftrape 146.

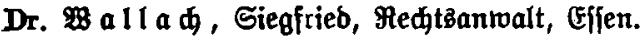

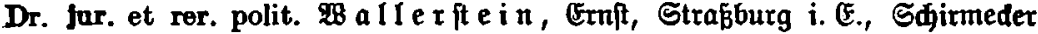
gting 15.

Dr. $\mathfrak{B}$ a ll ot, Redtäantwalt, Darmitabt, Mheinftr. 9 I.

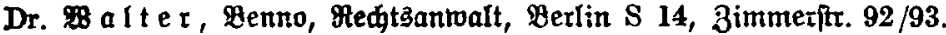

Dr. $\mathfrak{B}$ a e n ti, , \$., Brofefior, Salle a. S., Bambergerftr. 31.

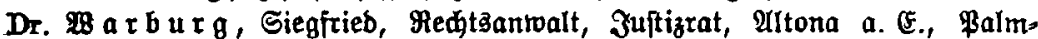
allee 31 .

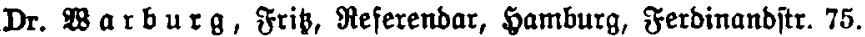

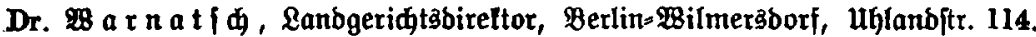

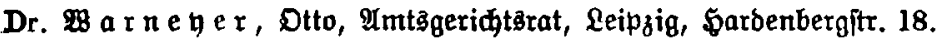

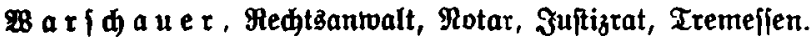

Dr. $\mathfrak{B}$ a r $\{$ d a u e $r$, Redt $3 a n$ walt, Bojen.

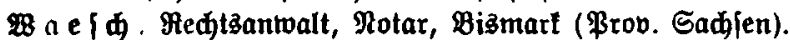

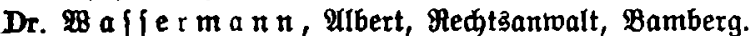

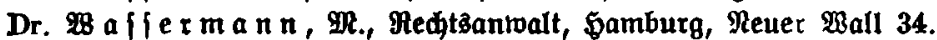

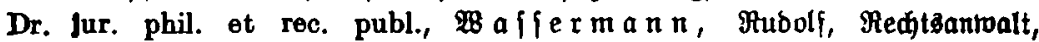
Betlin, friebridjtr. $120 \mathrm{I}$.

:

98 e $d$, Medtłantwalt, Diebenhofen.

28 e b e f in b, Lanogeriđtstat, Seh. Jultizrat, Danzig, Johlengalfe 5.

Dr. $\$$ e b e me yer, \$erner, ao. \$rofelfor ber Redte, Riel, Mbolfftr. 52 I

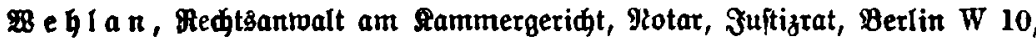

Tiergartenftr. 1.

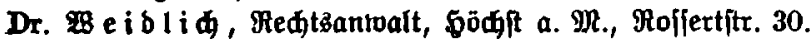

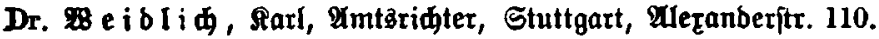




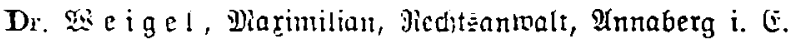

We i ge It, Medtsantoalt, Sotar, Eriurt, Anger 13 I.

Dr. Sa i igert, Crid, Sandriđter, Cottbuą.

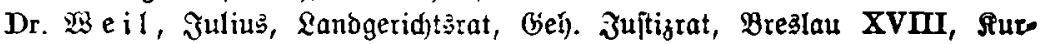
jüritenirrą̧e 37 .

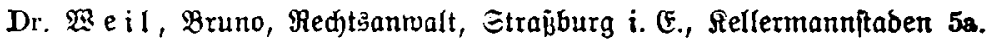

Dr. Seil, Stto, Grolih. Sotar, Seinheim (Baben).

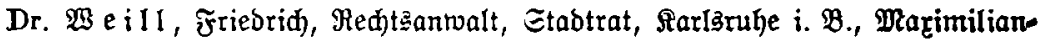
ftraje 6.

We i nberg, Rechtsantoalt, Berlin C 63, Rönigitr. 30.

Dr. $\mathfrak{B}$ e i n $\mathfrak{g} \mathfrak{a} \mathfrak{r} \mathrm{t}$, Eugen, Redtşantoalt, Mannheim.

Dr. $\mathfrak{B}$ e i $\bar{\xi}, \mathfrak{B i l h e l m , ~ R e d ̆ t s a n t w a l t , ~ B r e s l a u ~ I , ~ 3 r o i n g e r i t r . ~} 6$.

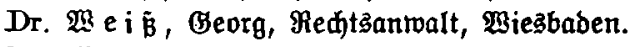

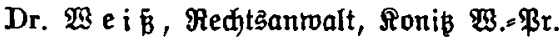

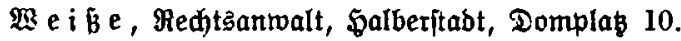

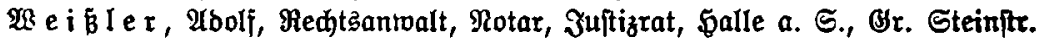

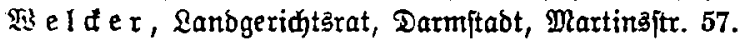

b e $\mathfrak{B}$ e I $\delta$ i g e, Urban, Medțanroalt, Juftizrat, Baberborn i. $\mathfrak{w}$.

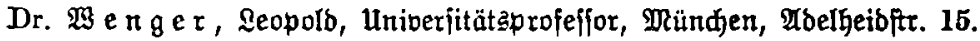

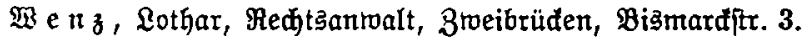

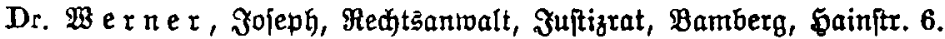

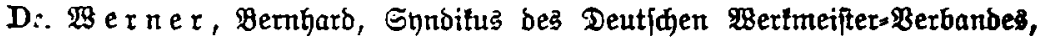
Düfielbori, Etromitr. 8.

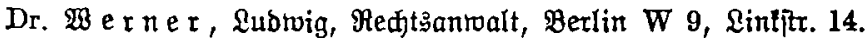

Si e r n e $\mathfrak{r}$, Emil, Redtsantwalt, Magbeburg, Raijerftr. 13.

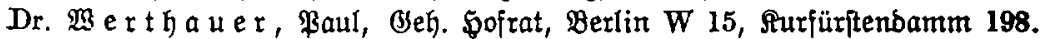

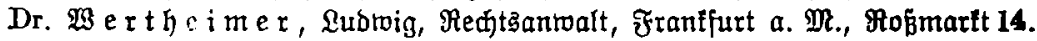

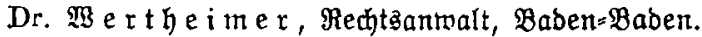

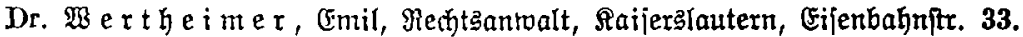

$2 \mathfrak{B}$ e $\mathrm{th}$ e i mer, Mr., Medjtsantvalt, Mündhen, Sarlsplą̧ 6 .

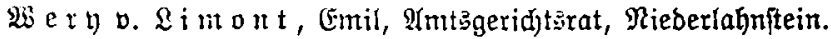

wi e ife I, Ređtşantwalt, Rotar, Juftizrat, Danzig, \$unbegajie 47 I.

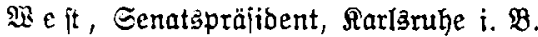

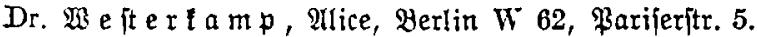

Yik e it m a n $n$, Redtiantwalt, Berlin SW 68, Ritteritr. $42 / 43$.

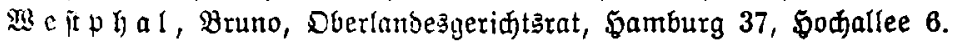

We e it $r$ u , Juftizrat, Ecllc.

Dr. $\mathfrak{B} \mathfrak{c}$ y $1, \Re$., Brofefior, Piel, Felditr. 111/113.

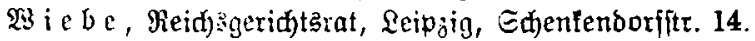

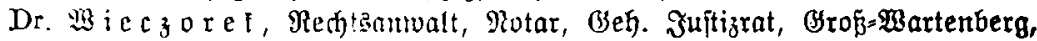
iłe 3 irt brestau.

$\mathfrak{X}_{3}$ i e lt

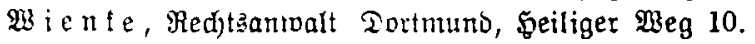

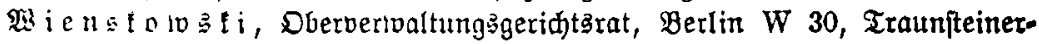
ftraje 7 .

2is i g a n b, Iandgeridtłłat, Wiesbaben, Mosbadjeritr. 14.

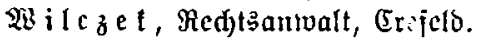




\section{LXVI}

Dr. 28 i l b h a ge $\pi$, Medtsantwalt, Juptizrat, Leipzig, Ferbinand Mhobeftr. 34.

פs il, Medtsantwalt, Thorn.

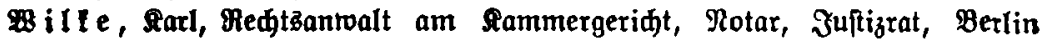
SW 61, Belle-glliance-Rlak 4.

v. 8 il mo vo $\mathbf{z} i$, Medt3antwalt bei bem Oberlanbeżgeridt, Megierung rat a. D., Juftizrat, Nauntburg a. S., Jenaeritr. 6a.

b on $\mathfrak{B}$ ilm, Aleranber, Bibilborjißgenber ber Erjałfommijfion, Bremen, Ryeinftr. 42.

28 i $1 \mathrm{~m} 3$, Medtsantwalt, Эuftizrat, (Erefelb.

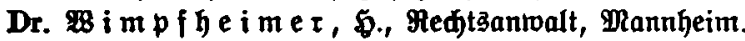

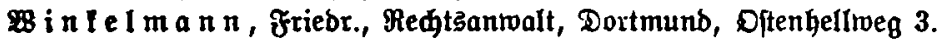

23 in 1 ler, Landgeridtsbirettor, (seh. Juftizrat, Erfurt, Serberftr. 31 I.

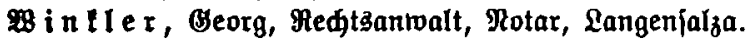

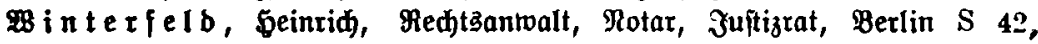
Dranienitr. 67.

$\mathscr{B} \mathfrak{i} \mathbf{r}_{z}$, Dberlandesgeridtşrat, Eolmar i. (E.

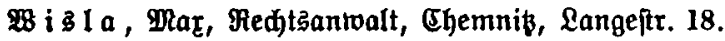

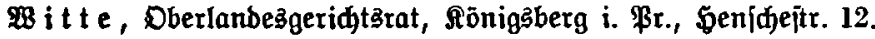

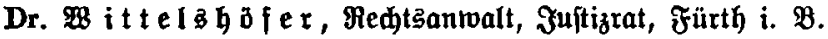

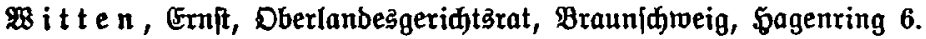

W it t e n ber $\mathfrak{B}$, Redtsanwalt, Bromberg.

Wit t e $r$, Max, Lanbgeridtsbireftor, Leipzig.

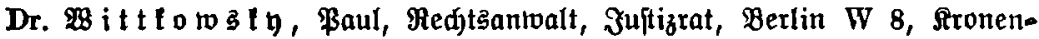
frafie 72.

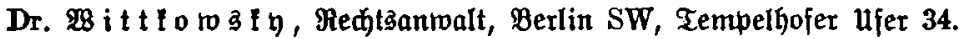

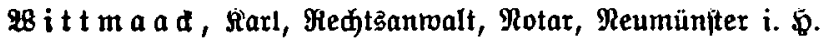

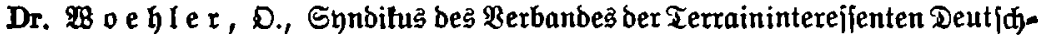
Ianbs, Berlin NW 7, Reue 2 ilheImitr. 12 II.

Dr. $\mathfrak{B}$ o $\mathfrak{h} \mathfrak{I} \mathfrak{a} \mathfrak{x} \mathfrak{h} \mathfrak{h}$, Theodor, Redt

Dr. 23 o h r iz e l, Emil, Fedtsanwalt, Leipzig, Betersftr. 28 III.

\$olbert, Redtzanivalt, Rotar, Juftizrat, Bot引bam.

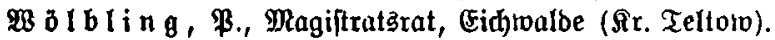

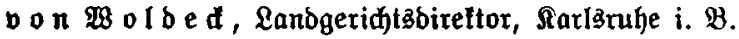

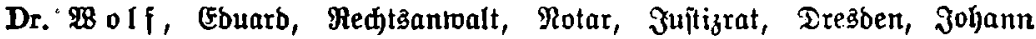
Beorgen=थllee 5 II.

Dr. $\mathfrak{B}$ o If , Redtantwalt, $\mathfrak{A l z h}$ i. Mheinhelien.

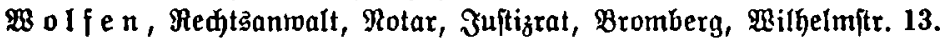

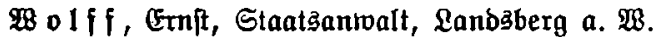

Dr. $\mathfrak{B}$ o If $f$, Martin, Brofeffor, Berlin= baleniee, balberftäbterftr. 2.

$\mathfrak{B}_{\text {olf }}, \mathfrak{F}_{\mathrm{ran}}$, Medtsantwalt, Rotar, Mülheim a. Muhr.

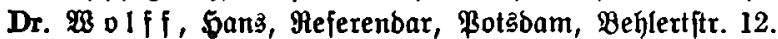

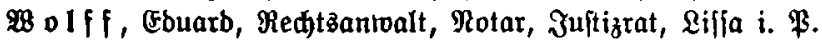

Dr. o If $f$, Max, Ređtsantwalt, Sifia i. .

Dr. $\mathfrak{Z}_{\text {o }}$ If $f$, Rechtsantwalt, Berlin W 8, Taubenftr. 36.

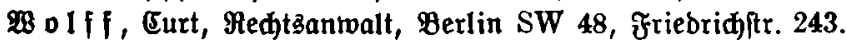

20 olff, Eugen, Ređtşantwalt, Juftizrat, Berlin W 57, Bülorwitr. 85.

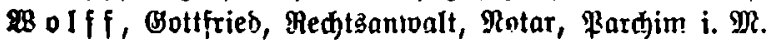




\section{LXVII}

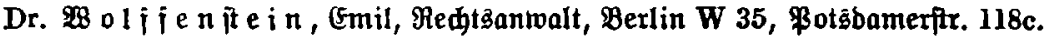

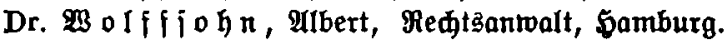

Wo If ram, Emil, Beh. Oberregierungezrat, vortragenber Fat in ber Oberrednungstammer, Potsbam, Eijengartitr. 8.

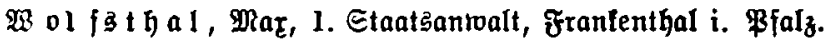

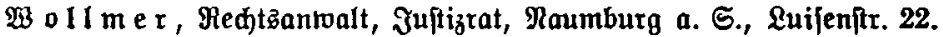

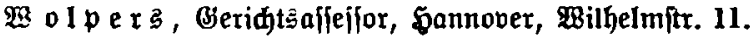

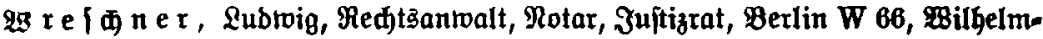
frafe 55.

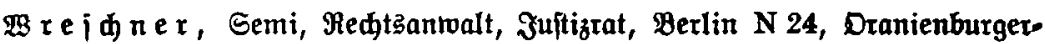
frape 33.

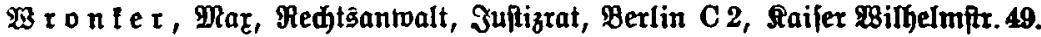

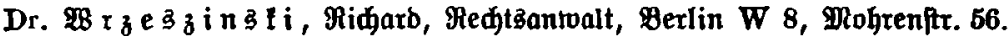

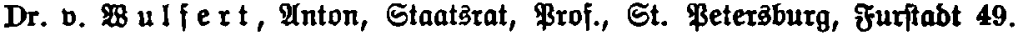
w u If

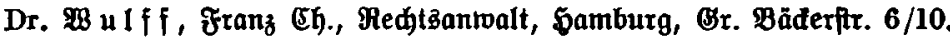

Dr. $\mathfrak{B} \mathfrak{u} \mathfrak{n} b$ e $\mathrm{l}$ i

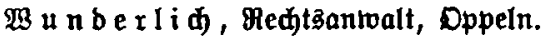

$\mathfrak{B}$ u n be r m a

$\mathfrak{B}$ u n be r m a de r, Martin, Redtzantwalt, Reujtabt 2 ejtpr.

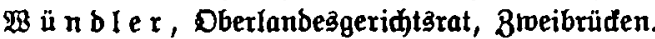

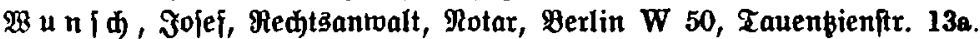

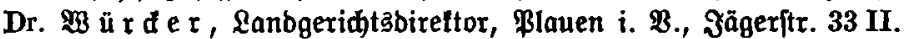

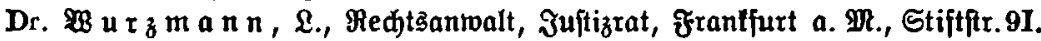

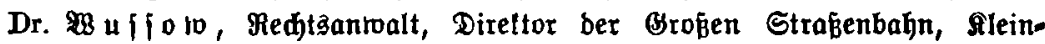
Mlienide, Briebnibitr. 6.

Dr. $\mathfrak{B}$ ü ft e n b ö r f e r, Profeffor, Roftơ i. M., Johann भIbredtftr. 14.

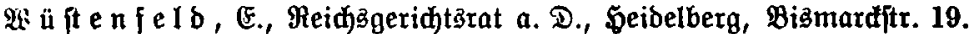

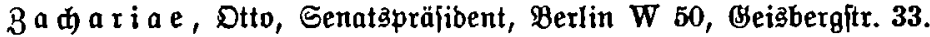

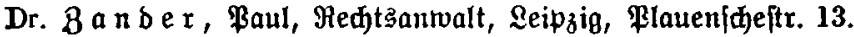

3 a n b e r, Redtzantwalt, Danzig, Langgaffe 20.

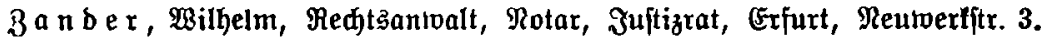

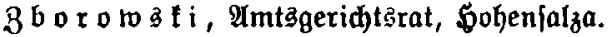

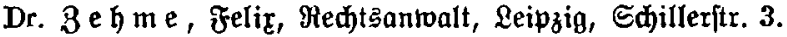

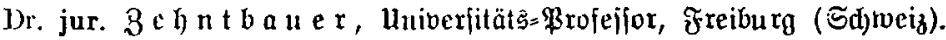

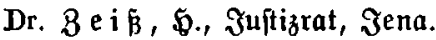

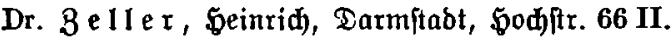

Be I te r, Redtłanmalt, Juftizrat, Stettin, Faijer Wilhelmftr. 2.

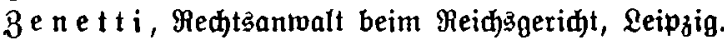

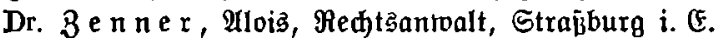

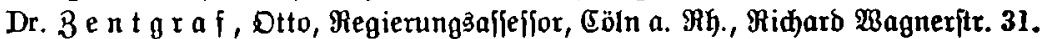

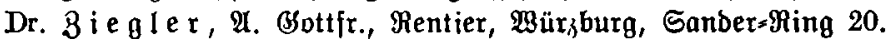

Dr. $B$ i e m\{fe $n$, F., Redtsantwalt, Berlin NW 6, \&uifenjtr. 35.

Dr. Bie fe, Redtsantualt, Motar, Jultizrat, Renogburg.

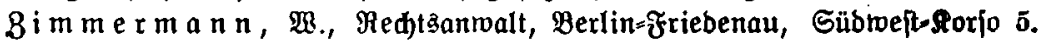
Binbel, Minifterialbirettor, Etuttgart. 


\section{LXYIII}

Dr. Birn o ftrá̉e 31.

Dr. 3 i t e l mann, Bleh. Juftizrat, Brofefior, Bonn, Eoblenzeritr. 83.

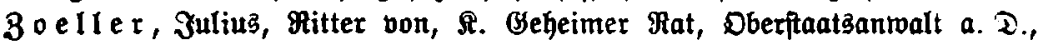

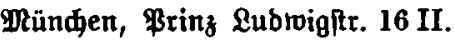

$3 \circ \mathfrak{p f f}$, Carl, Stabtrat, Leipzig.

Dr. 3 orn, Beh. Suftizrat, Brofeffor, Born.

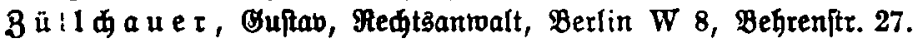

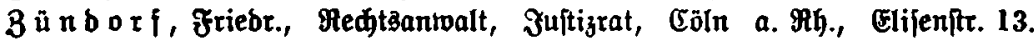

Dr. $\mathbf{B} \mathfrak{u} t$, Dlto, Rehtzantwalt, Farlstuke $i . \mathfrak{B}$.

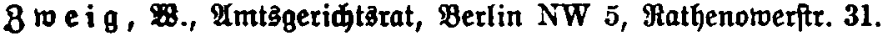

Dr. 3 we i gert, Dberreidsantwalt, Leipzig, \$Befftr. 4 .

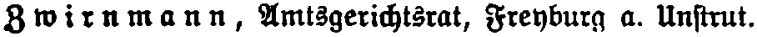

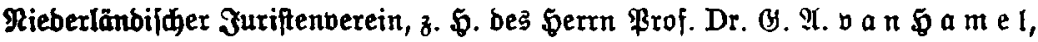
Ampterbam, 48 Ricolas $\mathfrak{B}$ itjentabe.

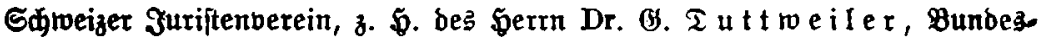
geridtstanżlei, Lau\{ante.

Leje- und Medehalle ber beutjđen Etudenten (Büderei=ßerwaltung) Prag.

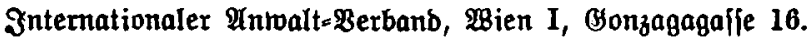

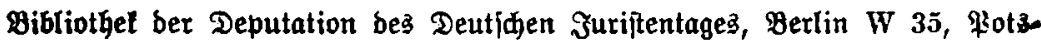
bamerftrape 118.

Eorre|ponberz Gerzberg, Berlin SW 48, Milhelmitr. 147.

Aönigl. Bibliothef Berlin.

Univerfitätåbibliothel $\mathfrak{B e r l i n . ~}$ 